\title{
Directional Wetting on Patterned Surfaces
}

\section{Olesya Bliznyuk}




\section{Committee members}

$\begin{array}{ll}\begin{array}{l}\text { Prof. dr. G. van der Steenhoven } \\ \text { (chairman) }\end{array} & \text { University of Twente } \\ \begin{array}{l}\text { Prof. dr. Ir. B. Poelsema } \\ \text { (promotor) }\end{array} & \text { University of Twente } \\ \begin{array}{l}\text { Dr. E. S. Kooij } \\ \text { (assistant promotor) }\end{array} & \text { University of Twente } \\ \text { Prof. dr. A. A. Darhuber } & \text { University of Einhoven } \\ \text { Dr. H. Wijshoff } & \text { Océ Technologies, Venlo } \\ \begin{array}{l}\text { Dr. R. M. van der Meer } \\ \text { Prof. dr. H. J. W. Zandvliet }\end{array} & \text { University of Twente } \\ \text { Prof. dr. A. van Silfhout } & \text { University of Twente } \\ & \text { University of Twente }\end{array}$

The work described in this thesis was carried out at the Physics of Interfaces and Nanomaterials group, MESA+ Institute for Nanotechnology, University of Twente, The Netherlands.

The research was financially supported by MicroNed, a national microtechnology program coordinated by the Dutch Ministry of Economic Affairs.

O. Bliznyuk

Directional wetting on patterned surfaces

ISBN: 978-90-365-3197-9

Published by Physics of Interfaces and Nanomaterials group, University of Twente

Printed by Gildeprint Enschede (C) O. Bliznyuk, 2011

No part of this publication may be stored in a retrieval system, transmitted, or reproduced in any way, including but not limited to photocopy, photograph, magnetic or other record, without prior agreement and written permission of the publisher.

\section{Cover}

Photo of morning dew droplets on Lupin leaf. (C)Anja Jonsson, 2011

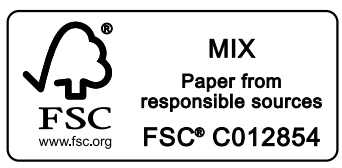




\section{DIRECTIONAL WETTING ON PATTERNED SURFACES}

\section{DISSERTATION}

to obtain

the degree of doctor at the University of Twente, on the authority of the rector magnificus, Prof. Dr. H. Brinksma, on account of the decision of the graduation committee, to be publicly defended on Thursday 7 July 2011 at 14:45 hrs

by

\section{Olesya Bliznyuk}

born on 19 October 1981

in Krasnodar, Russia 
The dissertation has been approved by:

Promotor: Prof. Dr. Ir. B. Poelsema

Assistant promotor: Dr. E. S. Kooij 


\section{Contents}

1 Introduction 1

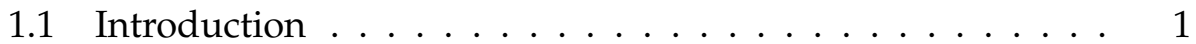

1.2 Wetting of the nozzle plate . . . . . . . . . . . 2

1.3 Chemically created anisotropic patterns . . . . . . . 5

1.4 Outline of the thesis . . . . . . . . . . . . . . . 6

2 Scaling of anisotropic droplet shapes on chemically stripe-patterned surfaces 9

2.1 Introduction . . . . . . . . . . . . . . . . 10

2.2 Experimental surfaces preparation . . . . . . . . . . . 10

2.3 Anisotropy due to surface pattern . . . . . . . . . . . 12

2.3.1 Static shapes of droplet footprints . . . . . . . . . 12

2.3.2 Directional dependent contact angles . . . . . . . . 14

2.4 Discussion . . . . . . . . . . . . . . . . . . 17

2.4.1 Spreading modes and final shape of droplets . . . 17

2.4.2 High and low surface tension liquid droplets . . . . . 19

2.5 Conclusions . . . . . . . . . . . . . . 22

3 Initial spreading kinetics of high-viscosity droplets on anisotro$\begin{array}{ll}\text { pic surfaces } & 25\end{array}$

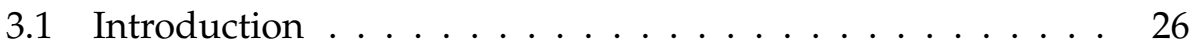

3.2 Experimental details . . . . . . . . . . . . . 26

3.2.1 Surface preparation . . . . . . . . . . . . . 26

3.2 .2 Droplet deposition. . . . . . . . . . . . . . . . 27

3.2 .3 High-speed camera . . . . . . . . . . . . . . . . . . . 28

3.3 Results ..................... 28

3.3.1 Kinetics of glycerol droplets . . . . . . . . . . . . . 28

3.3.2 Spreading kinetics dependence on surface chemistry 30

3.3 .3 First stage of spreading . . . . . . . . . . 32

3.4 Discussion . . . . . . . . . . . . . . . . . 34

3.4 .1 State of art ............... 34

3.4 .2 Inertial stage of spreading . . . . . . . . . . . 35

3.4.3 Capillary stage of spreading . . . . . . . . . . . . . 36

3.5 Conclusions . . . . . . . . . . . . . . . . . 39

4 Smart design of stripe-patterned gradient surfaces to control droplet $\begin{array}{ll}\text { motion } & 43\end{array}$

4.1 Introduction $\ldots \ldots \ldots \ldots \ldots \ldots \ldots$ 


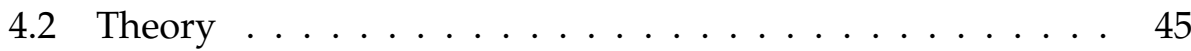

4.3 Experimental details . . . . . . . . . . . . . . 46

4.3 .1 Chemically created pattern . . . . . . . . . 46

4.3.2 Droplet deposition . . . . . . . . . . . . . . 48

4.3 .3 High speed camera . . . . . . . . . . . . . . . . . . . . . 49

4.4 Results ... . . . . . . . . . . . . . . . . 49

4.4 .1 Motion overview . . . . . . . . . . . . . . . . 49

4.4 .2 Fast camera results . . . . . . . . . . . . . 52

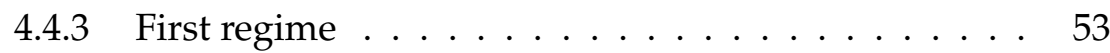

4.4 .4 Second regime $\ldots \ldots \ldots \ldots$

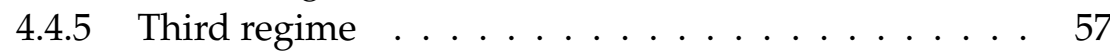

4.5 Discussion . . . . . . . . . . . . . . . 57

4.5.1 Capillary spreading over striped patterns: second and third regimes . . . . . . . . . . . . . 57

4.5 .2 Smart design . . . . . . . . . . . . 58

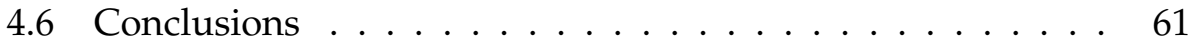

5 Droplet motion over radially patterned surfaces 63

5.1 Introduction . . . . . . . . . . . . . . . 64

5.2 Experimental details . . . . . . . . . . . . . . . . 65

5.2 .1 Surface preparation . . . . . . . . . . . 65

5.2 .2 Droplet deposition . . . . . . . . . . . . . 67

5.3 Results: Droplet motion overview . . . . . . . . . . . . 68

5.3.1 Advancing motion as compared to linear patterns . . 68

5.3.2 Droplets dewetting the radial pattern . . . . . . 70

5.4 Discussion . . . . . . . . . . . . . . . 72

5.4.1 Liquid bridges occurrence on radially patterned sur-

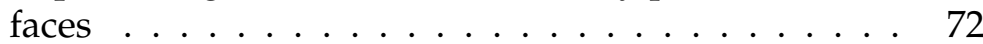

5.4.2 Kinetics of the receding motion . . . . . . . . . . 75

5.4.3 Surface energy dependence of the receding motion . 77

5.5 Conclusions . . . . . . . . . . . . . . . 78

6 Metastable droplets on shallow-grooved hydrophobic surfaces 81

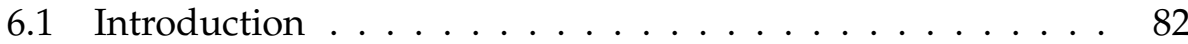

6.2 Experimental details . . . . . . . . . . . . . . . . . . 84

6.2.1 Surface preparation . . . . . . . . . . . . . 84

6.2 .2 Droplet deposition . . . . . . . . . . . . . . 85

6.3 Results ..................... 86

6.3.1 Droplet spreading parallel to the grooves . . . . . . 88

6.3.2 Droplet spreading perpendicular to the grooves . . . 92 


\section{CONTENTS}

6.4 Discussion . . . . . . . . . . . . . . . . . . 94

6.4.1 Final state of droplets: theoretical predictions . . . . . 94

6.4.2 Transition from metastable Cassie-Baxter to stable Wenzel ...................... 96

6.5 Conclusions . . . . . . . . . . . . . . . . . . 98

$\begin{array}{ll}\text { References } & 101\end{array}$

$\begin{array}{ll}\text { Summary } & 113\end{array}$

$\begin{array}{lr}\text { Samenvatting } & 119\end{array}$

$\begin{array}{ll}\text { Publications } & 125\end{array}$

$\begin{array}{ll}\text { Acknowledgements } & 127\end{array}$

$\begin{array}{ll}\text { About the author } & 129\end{array}$ 



\section{Introduction}

\subsection{Introduction}

In the present technological era computers and related appliances have become an important part of our everyday life. One of the numerous beneficial advantages is access to information from virtually any place we want. Another convenience of today's technology is the ability to transfer information from digital format onto paper for further usage via printers at home or at work. Inkjet printers, which use small droplets of ink to create an image on paper, represent a very popular choice for household application as well as industrial usage. The reliability and the high quality of the prints combined with the relatively low operational costs are the most important advantages of this specific category of printers.

However, document printing is not the only service drop-on-demand (DOD) printing technique has to offer [1-3]. Possible combination of inkjet technology with liquids other than conventional inks [4] enable new approaches in electronic circuit printing [5], manufacture of liquid crystalbased screens [6], displays [7], solar cells [8], data storage [9], optical fibers [10], drug dispensing [11], and many more. On the other hand, in order to further improve the printing speed and resolution, while simultaneously maintaining a high quality of the produced prints, the miniaturization of the inkjet head itself is required. To meet the demand, Micro Electro Mechanical Systems (MEMS) silicon-based technologies have been introduced and their further integration is being explored on an industrial level at increasingly large scales in the fabrication of inkjet head $[12,13]$.

Enhanced miniaturization poses new challenges to be solved, ranging from (i) improved ink channel design to (ii) development and optimization of piezo-materials to (iii) interference of the wetting layer on the nozzle plate with the firing of droplets. The goal of the work described in this thesis is to study possible solutions for problems imposed by the wetting 


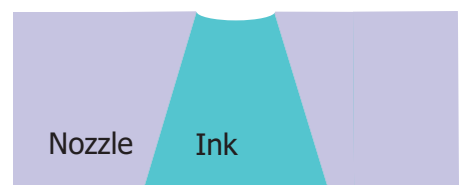

(a)

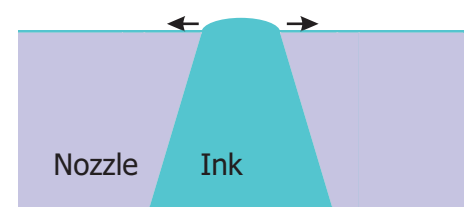

(b)

Figure 1.1: (a) Sketch of the nozzle filled with ink at rest before droplet firing starts. Ideally, the ink is fully confined within the nozzle. (b) Situation at rest is shown after a firing event. In this case, the surface of the nozzle plate is wetted by ink and the meniscus is protruding outside the nozzle. The pressure inside the ink channel results in a net ink flow onto the nozzle plate, increasing the ink layer thickness.

characteristics of the nozzle plate. More specifically, we aim at designing suitable patterned surface coatings to control the motion of the residual ink layer.

\subsection{Wetting of the nozzle plate}

The presence of a wetting layer of ink on the outer surface of the nozzle poses one of the major problems inhibiting reliable reproducible operation of the inkjet printhead [14-16]. Ultimately, this results in a deterioration of the print quality. More specifically, ink droplets are created by alternating negative and positive pressure waves inside the ink channel; this is achieved by a piezo-electric element integrated within the channel [3]. A key factor influencing the droplet firing process is the position of the airink interface. In order to assure a reliable and continuous jetting process, the triple contact line should be pinned at the edges of the nozzle, and the nozzle plate surface should remain ink-free, as schematically depicted in Fig. 1.1 (a). Ideally, the meniscus should remain inside the nozzle channel during the whole printing process.

However, in actual devices after a few seconds of firing the nozzle plate is covered by a micrometer-thick ink layer as is schematically represented in Fig. 1.1 (b). An actual image of a wetted nozzle plate is depicted in Fig. 1.2 (a) (the optically visible fringes arise from interference of light reflected on both sides of the wetting layer; their spacing indicates a thickness of the order of microns). The reason that inks readily wet the nozzle plate surface lies in the fact that most commercially relevant inks are based on low surface tension liquids exhibiting small contact angles. Thus, these liquids 


\subsection{WETTING OF THE NOZZLE PLATE}

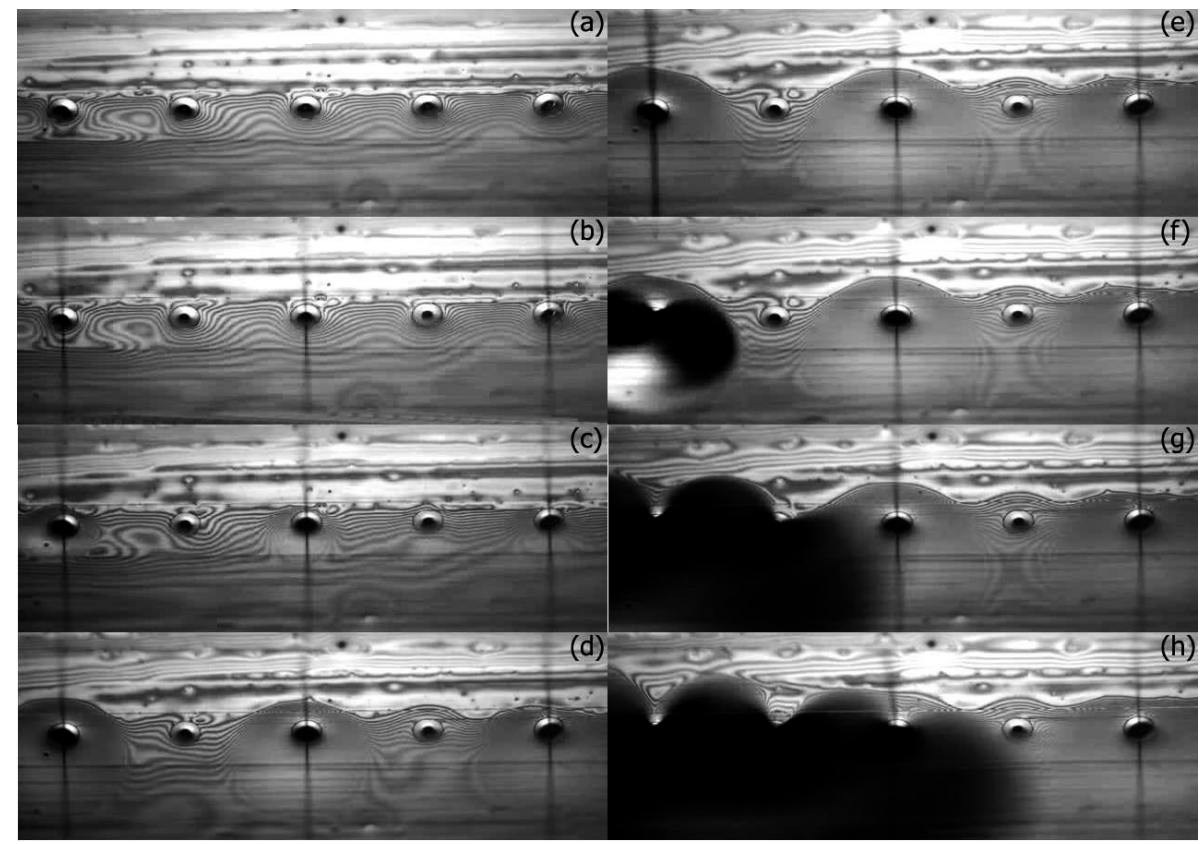

Figure 1.2: Influence of ink accumulation at the nozzle plate surface (ref. [17] ) on droplet jetting. (a) The nozzle plate prior the jetting, covered by a micrometer thick ink layer, as can be seen from the interference fringes. (b) Jetting of droplets is initiated from every second nozzles at $20 \mathrm{kHz}$. (c-d) Accumulation of ink near the nozzle occurs. (e) Left-most nozzle is about to stop firing. (f-g) The ink puddle increases as the channel remains under pressure. (h) The centre nozzle jetting is compromised: the droplet trajectory is altered.

attempt to minimize the surface energy by spreading and fully covering the entire available surface area. For the same reason, mechanical wiping is not sufficient to completely remove the wetting layer. Due to the presence of the ink layer, the triple contact line is not pinned at the nozzle edges. Nevertheless, jetting of droplets remains possible until a certain critical layer thickness is reached.

As a consequence of the depinning of the contact line as described above, the ink reservoir inside the print head is directly connected to the surface wetting layer. To enable firing of droplets, the ink in the channel is kept at a pressure which is higher than the ambient pressure on the outside. As a result, the ink is continuously being supplied to the surface wetting layer, therewith increasing its thickness (Fig. 1.1 (b) and Fig. 1.2 (c-e)). Once the thickness of the ink layer on the surface becomes larger than the aforemen- 


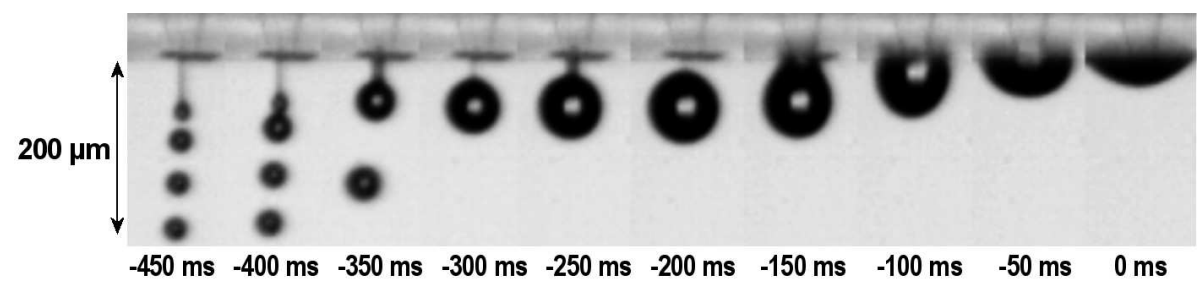

Figure 1.3: Failure of the droplet formation process during the last thousand drop formation cycles. As the drop speed decreases below a critical level, the adjacent drop will merge and a large amount of ink collects just below the nozzle opening. This large amount of ink is finally attracted to the nozzle plate and consequently leads to wetting of the nozzle plate [3].

tioned critical value, the nozzle will stop firing (Fig. 1.2 (f)). Moreover, once the droplet firing stops, the thickness of the wetting layer increases even faster, as larger quantities of ink are now directly supplied to the nozzle plate surface (Fig. 1.2 (g-h)). Eventually, the macroscopic ink 'puddle' reaches neighbouring nozzles which are still firing, resulting either in a change of the trajectory of ink droplets or leads to a complete blocking of the nozzles, therewith further compromising the print quality.

There are a number of ways the ink creates a wetting layer on the nozzle plate. First, mechanical and chemical defects in the vicinity of nozzle will promote depinning of the contact line which gives rise to ink wetting of the surrounding surface even prior to initiating the firing process. Second, as the frequency of droplet fire is typically around $30 \mathrm{kHz}$, the necessary channel refill during printing can also result in depinning of the contact line from the nozzle edges and give rise to overflow onto the nozzle plate. Another way would be the failure during droplet formation resulting in large size droplets attaching to the nozzle plate, as shown in Fig. 1.3.

The major requirement nowadays for drop-on-demand (DOD) inkjet printers is stability during firing process resulting in a high quality of the prints. As the presence of the wetting layer on the nozzle plate surface has profound consequences on the firing, a way to remedy the situation is to assure that the ink is constantly forced away from the nozzle. In order to facilitate the removal process, the wettability properties of the nozzle plate should be modified. More specifically, an antiwetting coating should be applied on which the ink will not form a film but will tend to form droplets. However, more extensive research is required focusing on the chemical stability of the coating in contact with the inks, as well as on wear resistance of potential coatings. 


\subsection{CHEMICALLY CREATED ANISOTROPIC PATTERNS}

Presently, on printheads manufactured using MEMS technology, the antiwetting coatings are applied homogeneously on the whole nozzle plate. Homogeneous coatings prevent the formation of a wetting layer but are not very effective against ink accumulation in the vicinity of the nozzle, thus requiring more sophisticated solutions.

\subsection{Chemically created anisotropic patterns}

The preceding discussion shows that inhibiting ink accumulation in the vicinity of the nozzle plate can considerably improve the overall printing quality. Homogeneous application of an antiwetting coating on the whole nozzle plate cannot assure removal of the ink from the nozzle. Furthermore, the ongoing miniaturization of the inkjet printheads prohibits integration of any active device that will assure that the ink is actively removed, for example by a temperature gradient.

A promising approach comprises the creation of a surface tension gradient by combining two chemical species with distinctly different surface energies. One should be more "hydrophobic" while the other should be "hydrophilic", i.e. non-wetting and wetting, respectively, for the ink used in the printing process. The overall surface tension should increase with increasing distance from the nozzle orifice. Ink droplets in contact with spatially varying surface energy on the nozzle plate will move in the direction of high surface tension and thus away from the nozzle.

A possible way to create such an energy gradient in a controlled way consists op applying a pattern made of stripes of alternating wettability, such as presented in Fig. 1.4. Changing the relative widths of stripes enables tuning the overall surface energy, while using a well-defined geometry creates a preferential direction for droplet motion parallel to the stripes. The advantage of such an anisotropic pattern as compared to a more simple, isotropic design consisting of a homogeneous hydrophobic circle around the orifice is that in the latter case the droplet only starts to move when it 'feels' the more wetting surface outside this circle. In the case of anisotropic patterns, motion should in principle be possible at any location within the pattern as long as the difference in surface energy on opposite sides of the droplet is large enough to initiate movement. 


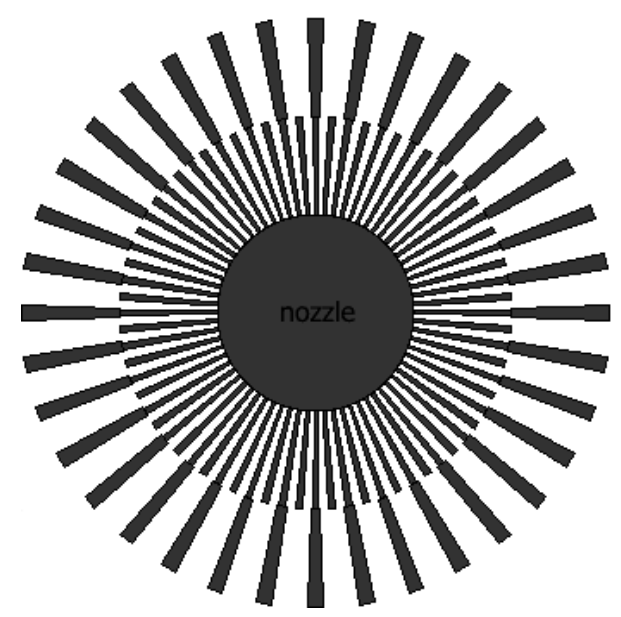

Figure 1.4: Sketch of a possible design for a chemically created pattern. The centre circle represents the nozzle orifice, the blue and white stripes correspond to two different chemical entities. Variations in overall surface energies are achieved by changing the relative widths of the hydrophilic/hydrophobic stripes.

\subsection{Outline of the thesis}

This thesis is organised as follows. In chapter 2 the static behaviour of droplets on chemically patterned surfaces consisting of parallel stripes of alternating wettability is presented. The overall surface energy is changed from predominantly hydrophobic to hydrophilic by variations of the relative stripe widths. The dependence of the static shape on the overall surface energy of the underlying pattern is discussed. Also, the behaviour of three different liquids with different surface tensions is compared. In chapter 3 the kinetics of deposition on the aforementioned stripe-patterned surfaces is described to enable a better understanding of the way the static shapes as studied in preceding chapter are achieved. Specifically, the evolution of the droplet and the motion of the contact line in orthogonal directions are compared. In the direction parallel to the stripes spreading is favourable, while perpendicular to the stripes the contact line experiences regularly spaced energy barriers posed by the hydrophobic stripes. Chapters 4 and 5 deal with droplets moving over a chemically defined surface energy gradient. More precisely, droplets move over pattern designs consisting of parallel stripes where the surface energy in increased upon moving over subsequent regions in the pattern. In chapter 4 we describe results pertaining to linear patterns. After a global description of the mo- 
tion of a droplet, preliminary comparative results are presented for patterns with different surface energy gradients. In chapter 5 the motion of liquid droplets over radially patterned surfaces is presented. This type of pattern is of prime interest from an application point of view, but the radially symmetric geometry of the design makes it more complicated for experimental studies. Consequently, the discussion is facilitated by comparison with the previously discussed linear patterns. Finally, in chapter 6 the static behaviour of droplets on shallow grooved, morphologically patterned substrates is considered. The patterns show clear similarities with those considered in chapters 2 and 3, although now instead of alternating hydrophilic/hydrophobic stripes, shallow grooves replace the hydrophilic part of the pattern. We compare the droplet characteristics on chemically and morphologically patterned surfaces in the Wenzel state. Moreover, on part of the microstructured surfaces the Cassie-Baxter state is observed. The origin of this 'fakir' state as well as the interdependence of the two observed states is discussed. 



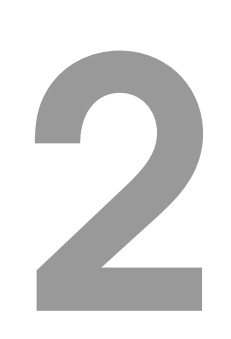

\section{Scaling of anisotropic droplet shapes on chemically stripe-patterned surfaces}

In this chapter an experimental study of droplet wetting behaviour on chemically patterned anisotropic surfaces is presented. Asymmetric static droplet shapes, arising from patterns of alternating hydrophilic (pristine $\mathrm{SiO}_{2}$ ) and hydrophobic (fluoroalkylsilane self-assembled monolayers) stripes with dimensions in the low-micrometer range, are investigated in relation to stripe width and separation. Owing to the well-defined small droplet volume, the static shape as well as the observed contact angles exhibit unique scaling behaviour. Only the relative width of hydrophilic and hydrophobic stripes proves to be a relevant parameter. 


\subsection{Introduction}

Controlling surface wettability is attracting significant scientific attention in many research areas, including fluid physics, materials science and interface physics. Applications of 'smart' surfaces with artificially designed wetting properties range, for example, from micro- or nanofluidic devices to car windows. Numerous theoretical and experimental studies have been conducted on chemically heterogeneous [18-22] and topographically structured surfaces [23-25].

Following the identification of surface roughness as the origin of the 'lotus effect' [26] and the race to pursue artificial superhydrophobic surfaces, most research has concentrated on investigating the behaviour of liquids on isotropic, topographically patterned surfaces [27]. Nevertheless, anisotropic surfaces can provide an important insight into the effect of dynamics on the final shape of the sessile droplet, therewith enabling one to gain a better understanding of the role of surface geometry. One of the first and nowadays still frequently studied anisotropic structured periodic surfaces consists of parallel grooves [28-32].

The investigation of similar anisotropic two-dimensional systems on morphologically flat substrates using chemical surface modification was hindered by the difficulty to reproducibly obtain sufficiently small features. Recent advances in the field of Self-Assembled Monolayers (SAMs) and their application for surface modification, combined with progress in lithographic patterning tools, enable reproducible manufacture of well-defined patterns to be used in wettability studies [33].

In this chapter we present an experimental study focused on chemically heterogeneous surfaces and their influence on the final shape of sessile drops. The well-defined, lithographically created patterns used in our experiments enable a detailed study and allow comparing of behaviour using different liquids. The observed anisotropic shapes as well as their quantification and scaling is discussed in view of the underlying patterns as well as liquid properties.

\subsection{Experimental surfaces preparation}

The surface patterns employed in our investigation consist of alternating hydrophobic and hydrophilic stripes (fluoroalkylsilane SAMs and bare $\mathrm{SiO}_{2}$ surface, respectively), giving rise to anisotropic wetting properties as schematically shown in Fig. 2.1. Using standard cleanroom facilities, silicon wafers with a thin layer of natural oxide are spin coated with positive pho- 
a)

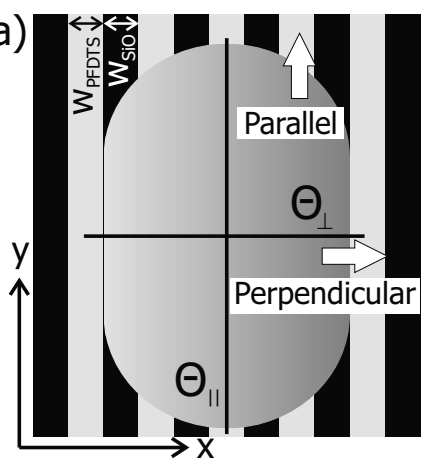

c)

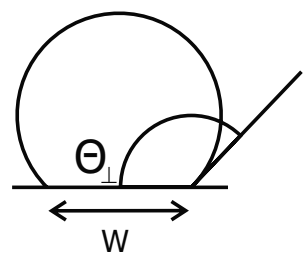

b)
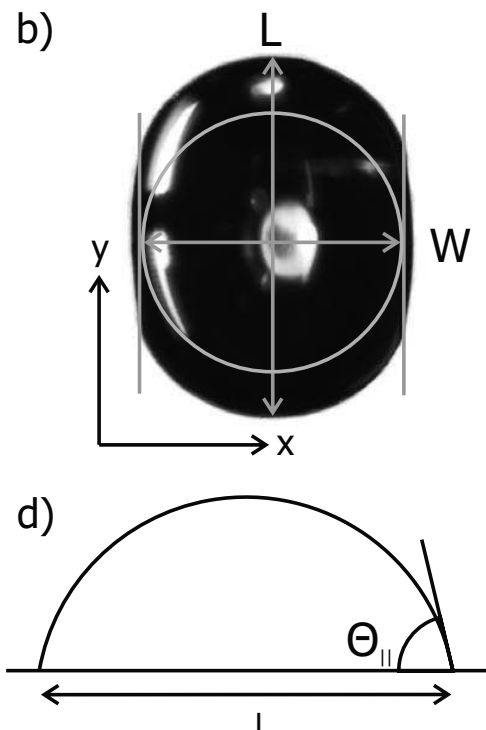

Figure 2.1: Quantifying anisotropic drops: (a) Schematic top-view representation and (b) photograph of an asymmetric (glycerol) droplet on a chemically patterned surface consisting of alternating $\mathrm{SiO}_{2}$ (hydrophilic) and $1 \mathrm{H}, 1 \mathrm{H}, 2 \mathrm{H}, 2 \mathrm{H}-$ PerFluoroDecylTrichloroSilane (PFDTS) (hydrophobic) stripes. The width of the stripes was varied in the range of $2-20 \mu \mathrm{m}$. The relative width of the stripes leads to a variation from a predominantly hydrophilic surface (wider $\mathrm{SiO}_{2}$ ) to mostly hydrophobic substrates (wider PFDTS). Two spreading regimes perpendicular and parallel to the stripes ( $x$ - and $y$-directions) lead to different macroscopic contact angles $\theta_{\perp}$ and $\theta_{\|}$and corresponding width $W$ and length $L$, as schematically depicted in (c) and (d), respectively. Note that the actual number of lines underneath the droplet is much larger than shown in (a).

toresist, enabling pattern creation via optical lithography. After the exposed photoresist is washed off, the remaining photoresist is baked and provides surface protection during vapor deposition of $1 \mathrm{H}, 1 \mathrm{H}, 2 \mathrm{H}, 2 \mathrm{H}$-perfluorodecyltrichlorosilane (PFDTS) (ABCR, Germany). After SAM formation the photoresist is washed off, leaving a chemically patterned surface.

The PFDTS molecule has a fluorinated chain consisting of 10 carbon atoms and a silane head-group that binds covalently to a thin layer of native oxide on the Si wafers. Once the chemical reaction between the silane head and the surface oxide has taken place, the fluorinated tail is exposed. The assembly of molecules creates a densely packed thin layer with a height in the order of one nm. Vapour deposition of the PFDTS molecules 
is done in a degassed chamber that is exposed in successive turns to PFDTS and water reservoirs to introduce the respective vapours, initiating the reaction on the wafer surface. Using a controlled environment ensures good quality and reproducibility of the SAMs [34].

Droplet deposition and characterization, including measurement of contact angles (CAs), is done using an OCA 15+ apparatus (Dataphysics, Germany). Droplets are created using a computer controlled syringe. Deposition of the droplet is achieved by very slowly lowering the suspended droplet onto the surface. As soon as the droplet is in contact with the surface, wetting induced spreading leads to detachment from the needle. For all droplets, the volume is fixed to $1 \mu 1$. As droplet dimensions are in the millimeter range, our surfaces are considered to be flat, but chemically heterogeneous [35]. Linewidths are varied in the range $2-20 \mu \mathrm{m}$; droplets span 700 to 80 lines. The liquids used are water (from Millipore Simplicity 185 system), glycerol (Glycerol ReagentPlus, SIGMA) and decanol (1Decanol for synthesis, MERCK Chemicals). The surface tensions of water and glycerol have similar values $\left(\gamma_{\mathrm{lv} \text {,wat }} \approx 72 \mathrm{~mJ} / \mathrm{m}^{2}\right.$ and $\left.\gamma_{\mathrm{lv}, \mathrm{gly}} \approx 65 \mathrm{~mJ} / \mathrm{m}^{2}\right)$. However, glycerol has a viscosity three orders of magnitude larger compared to water and also lower vapor pressure allowing prolonged measurements. Decanol has surface tension of $\gamma_{1 v, \text { dec }} \approx 28 \mathrm{~mJ} / \mathrm{m}^{2}$ and is used to study the behaviour of liquids with lower surface tensions on patterned surfaces.

The OCA 15+ enables determination of CAs with an accuracy below $0.5^{\circ}$; in all cases we performed multiple measurements. The experimental variations in CAs on identical sample were less than $2^{\circ}$. On our experimental unpatterned PFDTS SAMs we measured static CAs for water to be $\theta_{\mathrm{St}, \mathrm{wat}}=111^{\circ}, \theta_{\mathrm{St}, \mathrm{gly}}=106^{\circ}$ and $\theta_{\mathrm{St}, \mathrm{dec}}=59^{\circ}$. On pristine $\mathrm{SiO}_{2}$ respective static $C$ A values are $\theta_{\mathrm{St}, \mathrm{wat}}=45^{\circ}, \theta_{\mathrm{St}, \mathrm{gly}}=40^{\circ}$ and $\theta_{\mathrm{St}, \mathrm{dec}}=30^{\circ}$. An additional top-view camera is mounted to assess the in-plane droplet shape.

\subsection{Anisotropy due to surface pattern}

\subsubsection{Static shapes of droplet footprints}

The chemical pattern on our surfaces induces different spreading behaviour in orthogonal directions: spreading parallel to the stripes is favored at the expense of the contact line motion perpendicular to the stripes. This in turn gives rise to an equilibrium situation in which the droplet shape deviates from spherical, such as that shown in Fig. 2.1. In fact, the top-view of the droplets reveals that the shape can be approximated by a cylinder with 


\subsection{ANISOTROPY DUE TO SURFACE PATTERN}

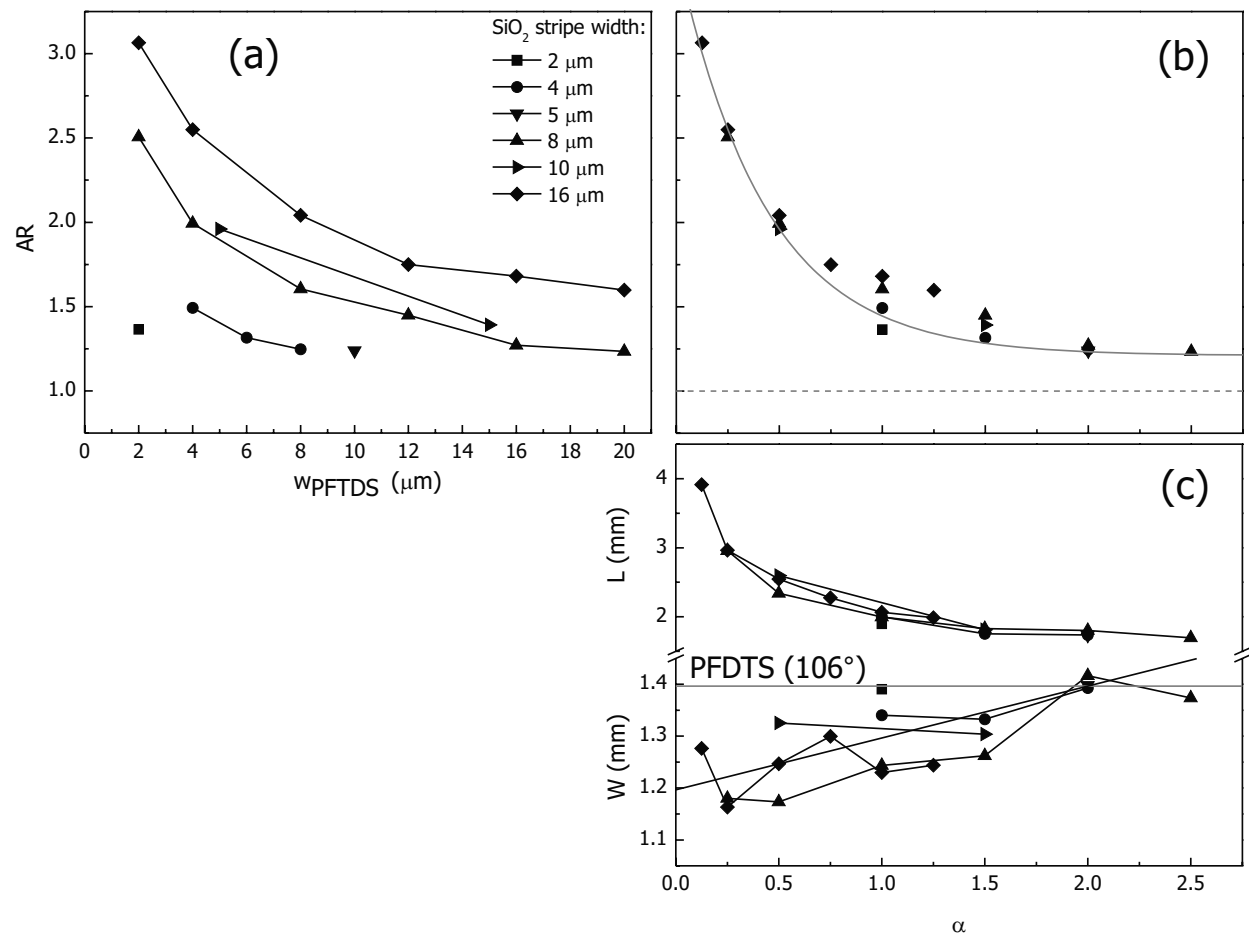

Figure 2.2: Experimentally determined glycerol droplet footprint parameters in relation to to the underlying chemical pattern. (a) Aspect ratio as a function of PFDTS stripe widths, for various $\mathrm{SiO}_{2}$ stripe widths. (b) AR falling on one line as a function of scaling parameter $\alpha$; the solid line is a guide to the eye. (c) Length $L$ and width $W$ of footprint reveal that the trend of AR is defined by $L$ behaviour. The horizontal gray line $(\approx 1.4 \mathrm{~mm})$ indicates the diameter of a spherical droplet of same volume $(1 \mu \mathrm{l})$ on unpatterned PFDTS for CAs as indicated.

two spherical caps. To quantify the distortion from a spherical shape, we introduce the aspect ratio $A R=L / W$, where $L$ represents the size parallel to the stripes and $W$ is defined as the width at the solid-liquid interface, as shown in Fig. 2.1.

Calculated ARs for glycerol droplets on our patterns are plotted in Fig.2.2 (a) showing that the droplet shape strongly depends on the relative width of the hydrophilic and hydrophobic lines. For fixed $\mathrm{SiO}_{2}$ stripe widths (connected symbols), the $A R$ increases markedly with decreasing PFDTS stripe widths; this is most pronounced for the largest $\mathrm{SiO}_{2}$ stripe widths experimentally studied.

To investigate whether the shape distortion is governed by a universal 
mechanism, we plot the $A R$ as a function of the relative hydrophobicity of each experimental surface. For that, we introduce the hydrophobic-tohydrophilic ratio, defined as a dimensionless parameter $\alpha$ :

$$
\alpha=\frac{w_{\mathrm{PFDTS}}}{w_{\mathrm{SiO}_{2}}}
$$

where $w_{\mathrm{PFDTS}}$ and $w_{\mathrm{SiO}_{2}}$ are the hydrophobic and hydrophilic stripe widths, respectively. In our experiments the ratio $\alpha$ varies between 0.125 and 6 , where $\alpha<1$ and $\alpha>1$ correspond to more hydrophilic or hydrophobic surfaces, respectively. For $\alpha=1$ the hydrophobic and hydrophilic areas are equal.

Fig. 2.2 (b) reveals that the $A R$ plotted as a function of $\alpha$ indeed leads to a single curve for all patterns. Apparently, the relative hydrophobicity, i.e. the ratio $\alpha$, is the predominant universal parameter defining the droplet shape. We stress that this only holds as long as the droplet spans many lines or the pattern dimensions remain in the micrometer range [36], i.e. variations of the liquid-vapor interface especially near the triple line can be neglected.

Clearly observable in Fig. 2.2 (b) is the pronounced decrease in the $A R$ with increasing $\alpha$ for more hydrophobic surfaces. For $\alpha>2.5$ (not shown in Fig. 2.2, see Fig. 2.4) the droplets appear almost spherical $(A R \approx 1)$.

Most surprisingly is the asymmetry, observed in Fig. 2.2. For $\alpha=2$ the exposed $\mathrm{SiO}_{2}$ area amounts to $1 / 3$ of the total area, giving rise to very limited anisotropy. On the other hand, for $\alpha=0.5$, at which the PFDTS-coated area amounts to $33 \%$, leads to highly asymmetric droplet shapes. Further reduction in the relative PFDTS-coated area leads to even more elongated droplets, with $A R \geq 3$. Note that for $\alpha=0$ (pure $\mathrm{SiO}_{2}$ surface), obviously, we observe perfectly spherical drop shapes with $A R=1$.

Furthermore, in Fig. 2.2 (c) the length $L$ and width $W$ are plotted as a function of $\alpha$. The length $L$ varies from $1.5 \mathrm{~mm}$ to $4.0 \mathrm{~mm}$, where the latter value (for $\alpha=0.125$ ) corresponds to the calculated droplet diameter on clean $\mathrm{SiO}_{2}$, while $W$ values are obtained within $0.3 \mathrm{~mm}$ of the theoretical value of $1.39 \mathrm{~mm}$ for clean PFDTS SAMs. This plot reveals that the elongation parallel to the stripes $L$ defines the $A R$ of the droplets, as the width variations are much smaller.

\subsubsection{Directional dependent contact angles}

Anisotropic wetting observed on stripe-patterned surfaces results in direction dependent static CAs, in agreement with static shapes deviating from 
(a)

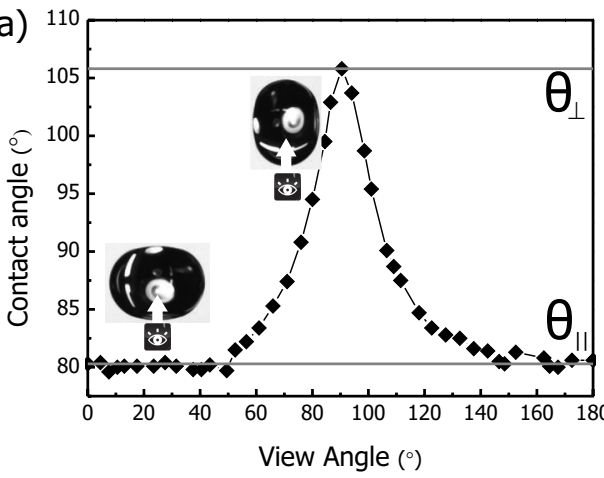

(b)

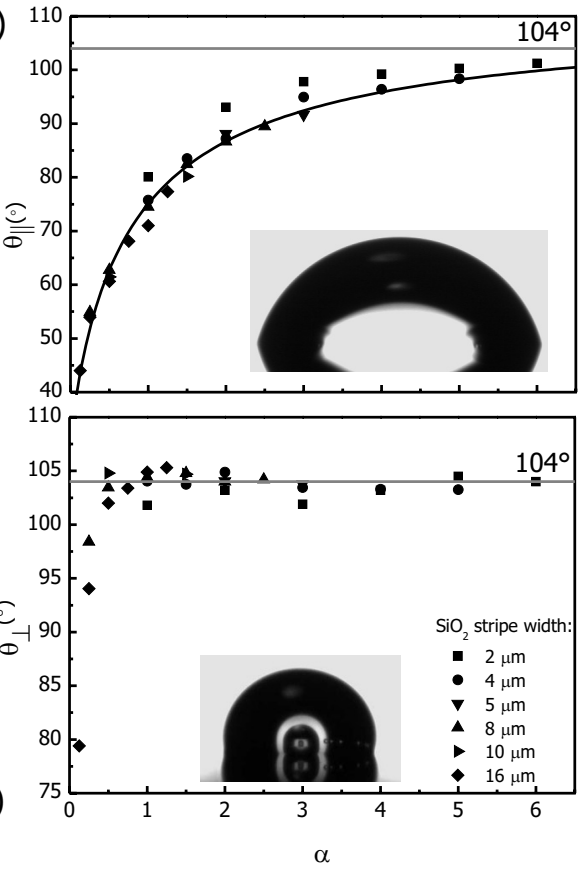

Figure 2.3: (a) CAs measured along the contour of a sessile glycerol droplet with $A R \approx 1.5$ on $8 \mu \mathrm{m} \mathrm{SiO}_{2} / 8 \mu \mathrm{m}$ PFDTS pattern; the inserts depict the limiting viewing angles, indicated by the white arrow. [(b) and (c)] CAs parallel $\left(\theta_{\|}\right)$and perpendicular $\left(\theta_{\perp}\right)$ to the stripe pattern as a function of $\alpha$. CAs scale to a single line: (b) $\theta_{\|}$is modeled by Cassie-Baxter equation (solid line) while (c) $\theta_{\perp}$ scatters around $104^{\circ}$.

spherical. Similar to the grooved surfaces, we observe the static CAs parallel to the stripes $\theta_{\|}$to have markedly smaller values compared to CAs measured perpendicular to the stripes $\theta_{\perp}$ (schematically shown in Fig. 2.1). As $\theta_{\|}$and $\theta_{\perp}$ result from two different regimes of spreading, these CAs represent two limiting values that can be measured on a droplet on a given surface. In order to illustrate the transition from $\theta_{\|}$to $\theta_{\perp}$, the CAs measured as a function of the horizontal viewing angle, i.e., around the contour of a glycerol droplet on a $50 \%$ hydrophilic- $50 \%$ hydrophobic surface are presented in Fig. 2.3 (a).

We choose to set a view angle of $0^{\circ}$ (consequently $180^{\circ}$ ) perpendicular to the stripes (indicated by the white arrows) when the CA parallel to the stripes $\theta_{\|}$is observed. At a view angle of $90^{\circ}$ the perpendicular CA $\theta_{\perp}$ is measured. Upon rotating the view angle from $0^{\circ}$ toward $90^{\circ}$, in this 
particular case up to $60^{\circ}$ the CAs have value of $80^{\circ}$ or $\theta_{\|}$, followed by the rapid increase until the maximum $C A$ value or $\theta_{\perp}$ is reached at $90^{\circ}$. The constant values in the view angle ranges of $0^{\circ}-50^{\circ}$ and $130^{\circ}-180^{\circ}$ show that along the circular parts of the droplet contour the CA is equal to $\theta_{\|}$. Only when the view angle of the droplet profile finds itself within $50^{\circ}-$ $130^{\circ}$ an increase in CAs is observed. In fact, the actual increase in the CA is much "sharper", but the fact that the entire droplet (and not only a crosssection) is viewed leads to a more gradually observed change. Finally, from the plot it is clear that the $\theta_{\|}$and $\theta_{\perp}$ correspond to the limiting CA values and are best suited to study spreading in orthogonal directions on striped surfaces.

The observed difference in values of $\theta_{\|}$and $\theta_{\perp}$ can be ascribed to the chemical composition of the substrate in the vicinity of the contact line. Specifically, in the direction parallel to the stripes, the droplet contour "feels" the rapidly varying hydrophilic/hydrophobic nature of the surface. As such, the static CA is expected to be mostly defined by the chemical composition of the surface underneath the droplet. Moreover, in contrast to $\theta_{\perp}$, $\theta_{\|}$is not a real CA, but an effective (macroscopic) CA, which is obtained by averaging the (microscopic) equilibrium CAs exhibited by the $\mathrm{SiO}_{2}$ and the PFDTS stripes [36, 37]. In this assumption, only the relative hydrophobicity, i.e., the value $\alpha$ in eq. 2.1, should influence the observed CA. Thus scaling behaviour is expected, similar to that in Fig. 2.2. The results in Fig. 2.3 (b) confirm that indeed the CAs fall onto a single curve, increasing from approximately $40^{\circ}$ at low $\alpha$-values toward $100^{\circ}$ at large $\alpha$-values.

Considering the droplet dimensions to be at least one order of magnitude larger than the pattern stripe widths, it is reasonable to consider that the droplet resides on an effectively (chemically) heterogeneous surface. In this case, the apparent CA is defined by the areal contribution of each species and the Cassie-Baxter equation $[38,39]$ can be used to model $\theta_{\|}$. For a binary composite surface the Cassie-Baxter equation is:

$$
\cos \theta_{C B}=f_{1} \cos \theta_{1}+f_{2} \cos \theta_{2}
$$

where $f_{1}$ and $f_{2}$ correspond to area fractions that exhibit CAs of $\theta_{1}$ and $\theta_{2}$, respectively. Considering the equilibrium CAs $\theta_{\mathrm{PFDTS}}$ and $\theta_{\mathrm{SiO}_{2}}$ on $100 \%$ PFDTS and $100 \% \mathrm{SiO}_{2}$ surfaces, respectively, and inserting the relative area fractions $\alpha /(1+\alpha)$ of PFDTS and $1 /(1+\alpha)$ for $\mathrm{SiO}_{2}$, rewriting eq. 2.2 yields a relation between $\theta_{\|}$and the scaling parameter $\alpha$

$$
\theta_{\|}=\arccos \left[\frac{\alpha \cos \left(\theta_{\mathrm{PFDTS}}\right)+\cos \left(\theta_{\mathrm{SiO}_{2}}\right)}{1+\alpha}\right]
$$




\subsection{DISCUSSION}

The $\theta_{\|}$in Fig. 2.3 (b) are fitted with eq. 2.3, using the static CAs on $\mathrm{SiO}_{2}$ and PFDTS as fitting parameters in order to achieve a better agreement between experimental and theoretical data. From the plot it is clear that the increase of $\theta_{\|}$from the lower limit for clean $\mathrm{SiO}_{2}$ to the upper limit for a PFDTS is adequately described using the Cassie-Baxter equation. The best fit for the experimental data was obtained for $\theta_{\mathrm{PFDTS}}=110^{\circ}$ and $\theta_{\mathrm{SiO}_{2}}=31^{\circ}$ (solid line in Fig. 2.3 (b)). The deviation from CAs measured on actual pristine surfaces $\left(106^{\circ}\right.$ and $40^{\circ}$, respectively) can be ascribed to small impurities, metastable $\theta_{\|}$(the droplet does not reach a global energy minimum, which would correspond to Cassie-Baxter CA) [40], and perhaps the very limited thickness variation in our patterns.

In Fig. 2.3 (c) $\theta_{\perp}$ as a function of the scaling parameter $\alpha$ is plotted. Contrary to $\theta_{\|}$most of the experimental $\theta_{\perp}$ scatter around a value of $104^{\circ}$; a slightly smaller value than the static CA for glycerol on a PFDTS monolayer $\left(106^{\circ}\right)$. Only for the lowest values of $\alpha$ in the range $0.125-0.25$ the observed CA is below $100^{\circ}$. Different behaviour from $\theta_{\|}$is expected in view of that $\theta_{\perp}$ is a real CA defined by a single chemical surface component and not the areal fractions of both. More precisely, straight segments of the contact line are pinned at the border between PFDTS and $\mathrm{SiO}_{2}$ stripes, the "last covered stripe" being $\mathrm{SiO}_{2}$. For all patterns studied, the contact line is probing the same situation irrespective of the absolute width of the stripes, which most likely is the reason for the similar $\theta_{\perp}$.

\subsection{Discussion}

\subsubsection{Spreading modes and final shape of droplets}

Previous studies of liquid motion on grooved and chemically patterned surfaces, can be briefly summarized as follows. For liquid advancing (or receding) in the direction parallel to the stripes, the contact line experiences a relatively small constant energy barrier. As a consequence, smooth continuous advancing motion of the droplet is observed until the static $\mathrm{CA}$ is achieved. In contrast, in the direction perpendicular to the stripes, the contact line will experience subsequent energy barriers, formed by the hydrophobic PFDTS stripes, giving rise to stick-slip-like motion $[18,35,41$ 43].

Furthermore, studies of the spreading reveal that initially the evolving droplet exhibits high contact angles, exceeding those enforced by the surface chemistry. For our surfaces, using a high-speed camera, we found that during the fast initial stage of spreading (on microseconds scale), the drop- 
lets spread equally fast in both directions. In the subsequent, much slower (on seconds scale) stage of spreading the influence of the underlying pattern can be seen. The droplet footprint gradually assumes an elongated shape by increasing its diameter parallel to the stripes $L$, while advancing perpendicular to the stripes no longer occurs ( $W$ remains constant) [44]. (This will be described in more details in the Chapter 3: Initial Spreading Kinetics of High-Viscosity Droplets on Anisotropic Surfaces).

Combining theory and experimental observations, the scaling behaviour observed for $A R$ and CAs can be addressed in more details. As the droplets reach their static values for footprint diameter and CA first in the direction perpendicular to the stripes, we first address the behaviour of $W$ and $\theta_{\perp}$ as a function of $\alpha$.

Simply put, the droplet will be able to spread perpendicular to the stripes as long as the actual CA will be greater than the advancing CA for PFDTS $\left(110^{\circ}\right)$ : the advancing $\mathrm{CA}$ on $\mathrm{SiO}_{2}$ is much lower. As the hydrophobic stripes are the limiting factor for advancing, it is reasonable to assume that the diameter of the wetted area cannot be larger than the one exhibited on unpatterned PFDTS. As a matter of fact, the experimental $W$ values scatter within experimental error around the aforementioned diameter for all patterns studied, showing that the hydrophilic part of the pattern has little influence on the static values. Furthermore, most of the CAs scatter around values of $104^{\circ}$ except for a few notable exceptions. The $2^{\circ}$ decrease compared to unpatterned PFDTS is most probably the result of elongation of the droplet footprint in the direction parallel to the stripes under the constraint of the finite droplet volume (fixed to $1 \mu \mathrm{l}$ ). Summarizing, for liquid advancing across a pattern consisting of well-separated chemical species, it the most hydrophobic part of the pattern that will define the final diameter as well as CA values.

As mentioned earlier, for spreading parallel to the lines the combination of both regions with different wettabilities define $\theta_{\|}$and, consequently, the elongation of the droplet necessary to reach the static CA value. Ideally, the droplet will continue to spread until the CA estimated by Cassie-Baxter equation (minimum free energy) in eq. 2.2 is reached. On actual surfaces used in our experiments the droplet will remain in one of the metastable states near the thermodynamic minimum, resulting in scatter of $\theta_{\|}$. Furthermore, for small values $\alpha<1$, the static CAs $\theta_{\|}$have low values, inducing important elongation of droplets. As we are using droplets of a fixed volume, the liquid is pulled away from the center region, resulting in lower droplets heights. Moreover, this accounts for the observed $\theta_{\perp}$ well below the maximum values of $104^{\circ}$ (Fig. 2.3 (c)): it is the macroscopic angle that 


\subsection{DISCUSSION}

is being measured, which is influenced by the droplet curvature due to the fitting procedure.

\subsubsection{High and low surface tension liquid droplets}

To investigate the variations in behavior of liquids with different properties such as surface tension, experiments have been performed on anisotropic surfaces with droplets of water, glycerol and decanol.

Both water and glycerol have comparably high surface tensions $\left(\gamma_{1 v \text {,wat }} \approx\right.$ $72 \mathrm{~mJ} / \mathrm{m}^{2}$ and $\gamma_{\mathrm{v}, \mathrm{gly}} \approx 65 \mathrm{~mJ} / \mathrm{m}^{2}$ ), so in principle similar behaviour is expected. Effectively, for $\alpha>0.5$ both $\theta_{\|}$and $\theta_{\perp}$ exhibit similar trends as can be seen in Fig. 2.4 (b) and Fig. 2.4 (c). More specifically, $\theta_{\perp}$ values scatter around a single value $\left(107^{\circ}\right.$ for water and $104^{\circ}$ for glycerol) while $\theta_{\|}$values scale to a single curve described by the Cassie-Baxter equation (eq. 2.2). For the Cassie-Baxter CA calculations, the experimentally measured CA values for PFDTS and $\mathrm{SiO}_{2}$ are used: no fitting has been done. The Cassie-Baxter equation predicts smaller values compared to experimental $\theta_{\|}$, particularly clearly observable for $\alpha>3$ : the difference can be explained in terms of Cassie-Baxter angles corresponding to global energy minima and $\theta_{\|}$values belonging to metastable states of higher energy. As for the $A R$, both liquids have similar trends falling on one line as a function of $\alpha$, though a water droplets show smaller $A R$ for all range of $\alpha$ values (Fig. 2.4).

However, for $\alpha<0.5$ there is a difference in static CA values and consequently in $A R$ for water and glycerol. In this range of $\alpha$ glycerol droplets show distinctly smaller values for $\theta_{\|}$and $\theta_{\perp}$ and consequently exhibit significant elongation parallel to the stripes, reflected in $A R>2$. The $A R \mathrm{~s}$ of water droplets however do not even reach a value of 2 . The origin for such different behavior for these patterns cannot be reasoned in terms of surface tension alone, which is confirmed by a slight difference in trends and values in case of $\alpha>0.5$. One thing that is clear so far from our experiments is that for water droplets the energy gained by further spreading and gaining energy by wetting hydrophilic $\mathrm{SiO}_{2}$ stripes is less compared to the energy needed to distort the droplet shape, resulting in static shapes as close to spherical as possible. In case of glycerol, on the contrary the energy gained by wetting extra hydrophilic surface is larger compared to the shape distortion, resulting in smaller CAs and $A R$ values larger than 2.

The fact that water droplets have much higher CAs and consequently lower $A R$ might be ascribed to a difference of liquid structures on the molecular level, such as hydrogen bonds. It is well known that being able to 
(a)

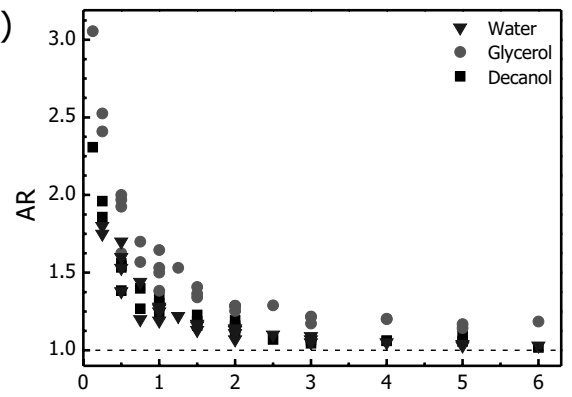

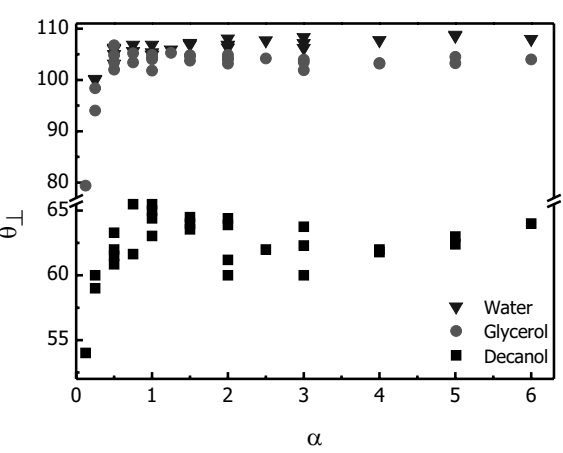

(b)

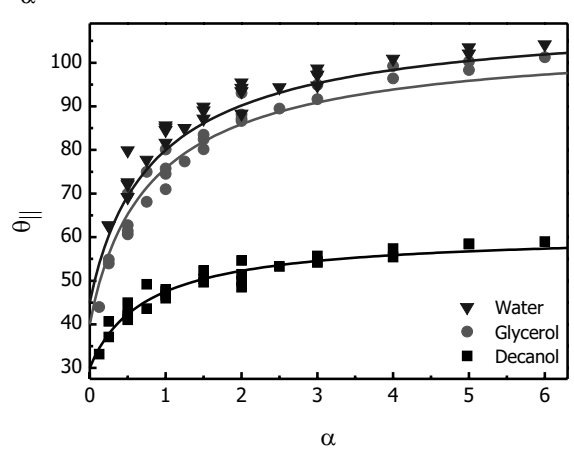

(c)

Figure 2.4: The experimental results for water, glycerol and decanol droplets are plotted as a function of universal scaling parameter $\alpha$. (a) Aspect ratios of droplets scale to three different curves. The order of the curves is contraintuitive: the highest belonging to glycerol and lowest to water. The scaling curves for (b) CAs perpendicular to the stripes $\theta_{\perp}$ and (c) CAs parallel to the stripes $\theta_{\|}$follow the order expected from surface tension arguments (from top to bottom): waterglycerol-decanol. The solid lines in (c) are theoretical CAs calculated using the Cassie-Baxter equation.

form four hydrogen bonds per molecule makes water such an outstanding liquid with amazing physical properties such as extremely high viscosity, boiling point, etc.[45, 46]. Glycerol molecule has three hydroxyl so is capable of three hydrogen bonds: just one short of water. However, the water molecule's hydrogen bonds are all intermolecular, strengthening the cohesion of liquid and requiring extra energy to be broken. On the contrary, in liquid state of glycerol, due to closed-up conformation of the molecules, intramolecular hydrogen bonds are formed, weakening the overall cohesion $[47,48]$. 


\subsection{DISCUSSION}

Based on this information, the difference in behavior between glycerol and water for $\alpha<0.5$ can be explained in the following way. In order to accommodate the low values of CA in the direction parallel to the stripes imposed by surface chemistry, an important distortion of shape from spherical is required which implies breaking a large amount of intermolecular hydrogen bonds. For water with 4 bonds per molecule, the energy required to break these is not compensated by wetting of $\mathrm{SiO}_{2}$ stripes, so water droplets do not reach the thermodynamic equilibrium CAs and remain in much higher metastable states. In case of glycerol, due to the smaller amount of intermolecular hydrogen bonds per molecule, less energy is required for breaking existing bonds and forming new ones with $\mathrm{SiO}_{2}$, resulting in droplets attempting to reach thermodynamic CAs predicted by the CassieBaxter equation.

In order to study the behaviour of liquids with lower surface tension, and consequently lower static CAs on both hydrophobic PFDTS and hydrophilic $\mathrm{SiO}_{2}$ decanol is used. Decanol is a relatively viscous long-chain alcohol, having a surface tension of $\gamma_{\mathrm{v} \text {,dec }} \approx 28 \mathrm{~mJ} / \mathrm{m}^{2}$. Experimental CAs of $59^{\circ}$ and $30^{\circ}$ are observed on pure PFDTS and $\mathrm{SiO}_{2}$ surfaces respectively. Nevertheless, deposited on our stripe patterned surfaces, static cylindrical shapes similar to those exhibited by glycerol and water are found.

Plotting the experimental $\theta_{\perp}$ and $\theta_{\|}$for decanol as a function of $\alpha$ again shows scaling behaviour (Fig. 2.4 (b) and Fig. 2.4 (c)). Surprisingly, $\theta_{\perp}$ for almost all patterns scatter around $63^{\circ}\left(4^{\circ}\right.$ above the static CA on unpatterned PFDTS; both water and glycerol scatter around CAs few degrees smaller than the CA on PFDTS). Only the $\theta_{\perp}$ for the smallest $\alpha=0.125$ has a value which is $10^{\circ}$ smaller. As with the high surface tension liquids, the trend of $\theta_{\|}$can be modeled by the Cassie-Baxter equation (eq. 2.3). Again, similar to water and glycerol, in case of $\alpha>3$ the model predicts smaller values compared to experimental ones.

When the $A R$ values for decanol are plotted as a function of $\alpha$, the data also scales to one line as shown in Fig. 2.4 (a). In contrast to expectations, the scaling curve lies between those for water and glycerol, and not below them as may be expected based on surface tension arguments. Moreover, $A R$ values above 2 are measured, while the maximum for water does not exceed 1.8. In order to propose an explanation for the relative shifting of scaling lines, experiments with more liquids having various surface tensions are to be carried out. 


\subsection{Conclusions}

We have investigated the three-dimensional shape of droplets deposited on anisotropic chemically heterogeneous surfaces, formed by alternating hydrophilic and hydrophobic stripes with widths in the low-micrometer range. Three liquids having different properties are studied: water and glycerol are used as example of high surface tension liquids while decanol is used to investigate the behaviour of relatively low surface tension liquids. For all three liquids the aspect ratio of the droplets as well as the contact angles in directions parallel and perpendicular to the stripes exhibit remarkable scaling behaviour as a function of relative hydrophobicity of the underlying surface. As long as the droplet dimensions are one to two orders of magnitude larger than the width of the stripes, these quantities do not depend on the absolute size of the surface pattern, but only depend on the relative width of the hydrophobic and hydrophilic stripes. 




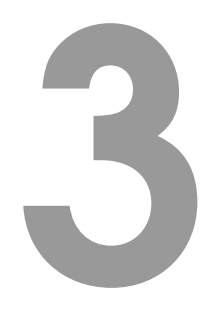

\section{Initial spreading kinetics of high-viscosity droplets on anisotropic surfaces}

Liquid droplets on chemically patterned surfaces consisting of alternating hydrophilic and hydrophobic stripes exhibit an elongated shape. To assess the dynamics during droplet formation we present experimental results on the spreading of glycerol droplets on such surfaces using a high-speed camera. Two spreading regimes are observed. Initially, in what is referred to as the inertial regime, the kinetics is dominated by the liquid, and spreading is only weakly dependent on the specific surface properties. As such, liquid spreading is isotropic and the contact line maintains a circular shape. Our results reveal a remarkably long inertial regime, as compared to previous results and available models. Subsequently, in the viscous regime, interactions between the liquid and underlying pattern govern the dynamics. The droplet distorts from a spherical cap shape to adopt an elongated morphology that corresponds to the minimum energy configuration on stripe-patterned surfaces. 


\subsection{Introduction}

Wetting of solid surfaces by liquids was and is attracting considerable attention in the scientific community by the apparent simplicity on one hand and the amount of unsolved fundamental and experimental challenges on the other hand. The diversity of the field brings together physics, chemistry, engineering and mathematics in an attempt to gain a full understanding of phenomena occurring in our everyday life. Other than being of purely scientific interest, wetting behaviour is a crucial part of many industrial processes ranging from pesticide deposition [49] to inkjet printing [3]. For numerous applications wetting control is of utmost importance for quality improvement as well as making processes more economically and ecologically profitable.

It is generally recognized that both interface chemistry and surface structure are essential parameters which determine static and dynamic wetting behaviour $[23,50,51]$. Chemical modifications of flat surfaces to alter the wetting properties are of particular interest from fundamental as well as applications point of view, the self-assembled molecular monolayers (SAMs) being particularly popular [33, 42, 52]. By far the most widely studied pattern on various length scales consists of parallel stripes of alternating wettabilities due to well-defined anisotropic wetting behaviour [18, $19,22,35,43]$.

In this chapter, we present an experimental study into the spreading of glycerol droplets in case of partial wetting using a high-speed camera. In the first part, differences in the evolution of the droplets induced by variation of the underlying pattern are presented and discussed. In the second part, a more detailed study of the initial moments of the spreading dynamics is described. The results are discussed in the perspective of existing models for dynamics on short time scales.

\subsection{Experimental details}

\subsubsection{Surface preparation}

The surface patterns employed in our experimental studies consist of alternating hydrophobic and hydrophilic stripes on silicon wafers: fluoroalkylsilane SAMs (PFDTS) and bare $\mathrm{SiO}_{2}$ surface, respectively. Such patterns give rise to anisotropic wetting properties in orthogonal directions as shown in Fig. 3.1. As droplet dimensions are in the millimetre range while the pattern widths are typically of the order of several micrometers, our surfaces 


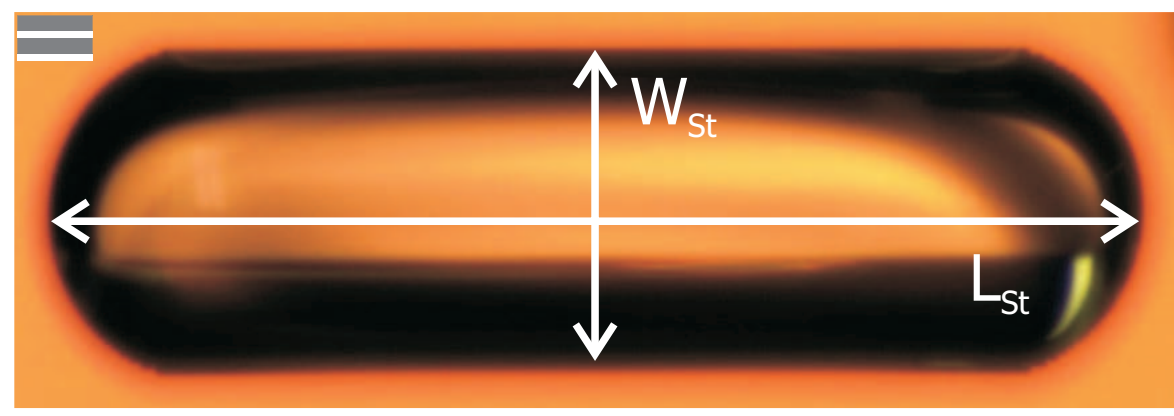

Figure 3.1: Top view image of a sessile glycerol droplet on our experimental surface. The static shape on the pattern having stripe widths of $16 \mu \mathrm{m} \mathrm{\textrm {SiO } _ { 2 }}$ (hydrophilic) and $2 \mu \mathrm{m}$ PFDTS (hydrophobic) is presented. In order to quantify the relative hydrophobicity of the patterns we introduce the dimensionless parameter $\alpha$ given by hydrophobic-to-hydrophilic line width ratio $\left(\alpha=w_{\mathrm{PFDTS}_{\mathrm{S}}} / w_{\mathrm{SiO}_{2}}\right)$. To quantify the distortion from a spherical shape, we introduce aspect ratio $A R=L_{\mathrm{St}} / W_{\mathrm{St}}$, where $L_{\mathrm{St}}$ and $W_{\mathrm{St}}$ are diameters parallel and perpendicular to the stripes, respectively. The $A R$ of the presented droplet amounts to 3.3.

are considered to be flat, but chemically heterogeneous [35]. The way our experimental surfaces are prepared is discussed in detail in the experimental section of Chapter 2 .

\subsubsection{Droplet deposition.}

Droplet deposition and characterization, including measurements of contact angles (CAs), is done using an OCA 15+ apparatus (DataPhysics, Germany). The liquid used is glycerol (ReagentPlus, Sigma, USA). For all droplets the volume is fixed at $3 \mu \mathrm{l}$; the variation in droplet diameter just after being produced from the syringe was measured to be less than $5 \%$. Before deposition on the surface, the droplet is allowed to rest for approximately 1 minute to reduce possible vibrations due to the formation process. Deposition of the droplet is achieved by very slowly lowering the syringe with the suspended droplet until it contacts the patterned surface. The velocity of approach is measured to be lower than $5 \mathrm{~mm} \cdot \mathrm{s}^{-1}$ in order to minimize the kinetic energy influence on the spreading of droplets [53].

Due to the relatively small volume of the droplets in our experiments, the gravitational influence can be neglected and liquid-solid interactions dominate the behaviour of the liquid. Once the droplet is in contact with the surface, wetting-induced spreading leads to detachment from the nee- 
dle, after which the final shape is reached generally after a few seconds owing to the large viscosity of glycerol $(\eta \approx 1.4 \mathrm{~Pa} . \mathrm{s})$.

\subsubsection{High-speed camera}

The shape evolution is determined from images taken using a Photron SA3 high speed camera at $15000 \mathrm{fps}$; the camera is operated using Photron Fastcam Viewer 3 software. For each pattern, movies of droplet spreading were taken for both directions parallel and perpendicular to the stripes. Prior to capture of the movie, a $3 \mu 1$ glycerol droplet is suspended a few millimetres above the substrate, while attached to the needle. The capture is started simultaneously with the lowering motion and care is taken that the first $1.8 \mathrm{~s}$ of spreading are captured. Due to the high viscosity of glycerol and the strong elongation for patterns with small values for $\alpha$, the time to reach equilibrium exceeds the available camera memory. The diameter of the droplets in both directions was determined with an accuracy of $0.05 \mathrm{~mm}$ using a MATLAB program.

\subsection{Results}

\subsubsection{Kinetics of glycerol droplets}

In Fig. 3.2 we present fast camera image frames from a sequence that was taken for a surface with $\alpha=0.125$, i.e. for the most hydrophilic pattern experimentally studied. The first frame (Fig. 3.2 (a)) depicts the suspended droplet; once the droplet is in equilibrium, it is slowly lowered onto the surface. The time count starts the moment the droplet touches the surface, which can be defined within a $140 \mu$ s range (Fig. 3.2 (b)). The third and fourth frames (Fig. $3.2(\mathrm{c}, \mathrm{d})$ ) show the droplet while the contact area with the chemically patterned surface rapidly increases. In this time interval, the volume is considerably rearranged while the liquid centre of mass moves down toward the substrate. On the fifth and the sixth frames (Fig. $3.2(\mathrm{e}, \mathrm{f})$ ) a growing elongation in the direction of the stripes is depicted. As the wetted area increases, more of droplet volume is entrained towards the surface, thinning the neck connecting the droplet to the needle (Fig. 3.2 (f)). After 188ms (Fig. 3.2 (g)), the neck breaks and the droplet detaches completely from the needle. The detachment moment is accompanied by a jump in the wetted area as well as in the CA evolution: during a brief period of less than $100 \mathrm{~ms}$ the shape rearrangement is enhanced. On the last snapshot, the droplet is shown $110 \mathrm{~ms}$ after the detachment, once the 
(a)

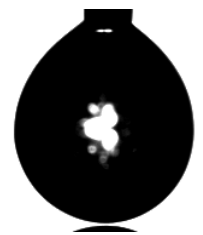

(e)

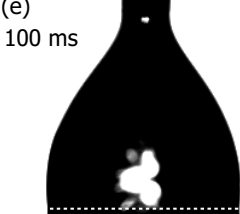

(b)

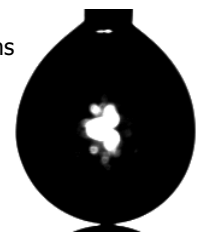

(f)

$150 \mathrm{~ms}$

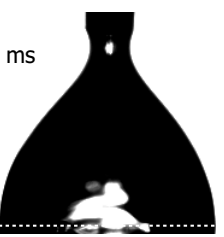

(c)

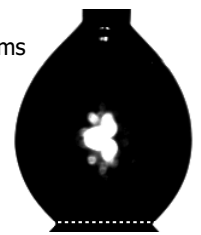

(g)

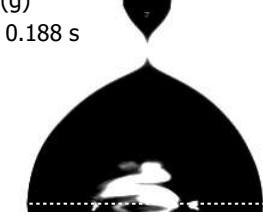

(d)

$50 \mathrm{~ms}$

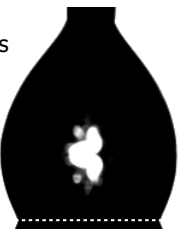

(h)

$0.3 \mathrm{~s}$

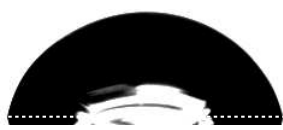

Figure 3.2: Fast camera snapshots showing the spreading of a $3 \mu 1$ glycerol droplet on a chemically patterned Si wafer in the direction parallel to the stripes. The pattern consists of alternating $16 \mu \mathrm{m} \mathrm{SiO}_{2}$ (hydrophilic) / $2 \mu \mathrm{m}$ PFDTS (hydrophobic) stripes, i.e. with $\alpha=0.125\left(\theta_{\|}=53^{\circ}\right)$. The dash-dotted line indicates the surface. (a) The droplet is suspended at rest prior to deposition $(R=0.95 \mathrm{~mm})$; (b) the moment the contact with the surface takes place; (c), (d) snapshots from the fast stage of spreading; (e), (f) snapshots from the slow stage of spreading: the shape of the contact line is progressively becoming an ellipse as elongation in the direction of the stripes takes place; $(\mathrm{g})$ the droplet detaches from the needle; (h) droplet $0.1 \mathrm{~s}$ after the detachment from the needle. In the following, the droplet will evolve on the timescale of seconds to its final shape.

rapid evolution of both diameters has stopped. Subsequently, the droplet will slowly evolve to reach its final shape, taking between $2 \mathrm{~s}(\alpha=2.5)$ and $10 \mathrm{~s}(\alpha=0.125)$. These large timescales are attributed to the high viscosity of glycerol.

In order to illustrate the changes in the droplet footprint evolution as a function of the underlying chemistry, in Fig. $3.3(\mathrm{a}, \mathrm{b})$ the length $(L(t)$, the diameter of wetted area parallel to the stripes) and the width $(W(t)$, the diameter perpendicular to the stripes) are plotted versus time. The two values of $\alpha$ chosen pertain to the most hydrophilic and the most hydrophobic patterns studied.

Plotting the diameter growth against time reveals that the fastest evolution kinetics occurs on the surface with the smallest hydrophobic fraction in the pattern (Fig. 3.3 (a)). Furthermore, the most pronounced difference in growth of $L(t)$ and $W(t)$ is present for this $\alpha=0.125$ (the final aspect ratio is close to 3). On the pattern with $\alpha=2.5$ (Fig. $3.3(\mathrm{~b})$ ), the evolution of $L(t)$ and $W(t)$ exhibits slower kinetics. The growth of the two diameters shows only small differences, in agreement with the nearly spherical final shape. 

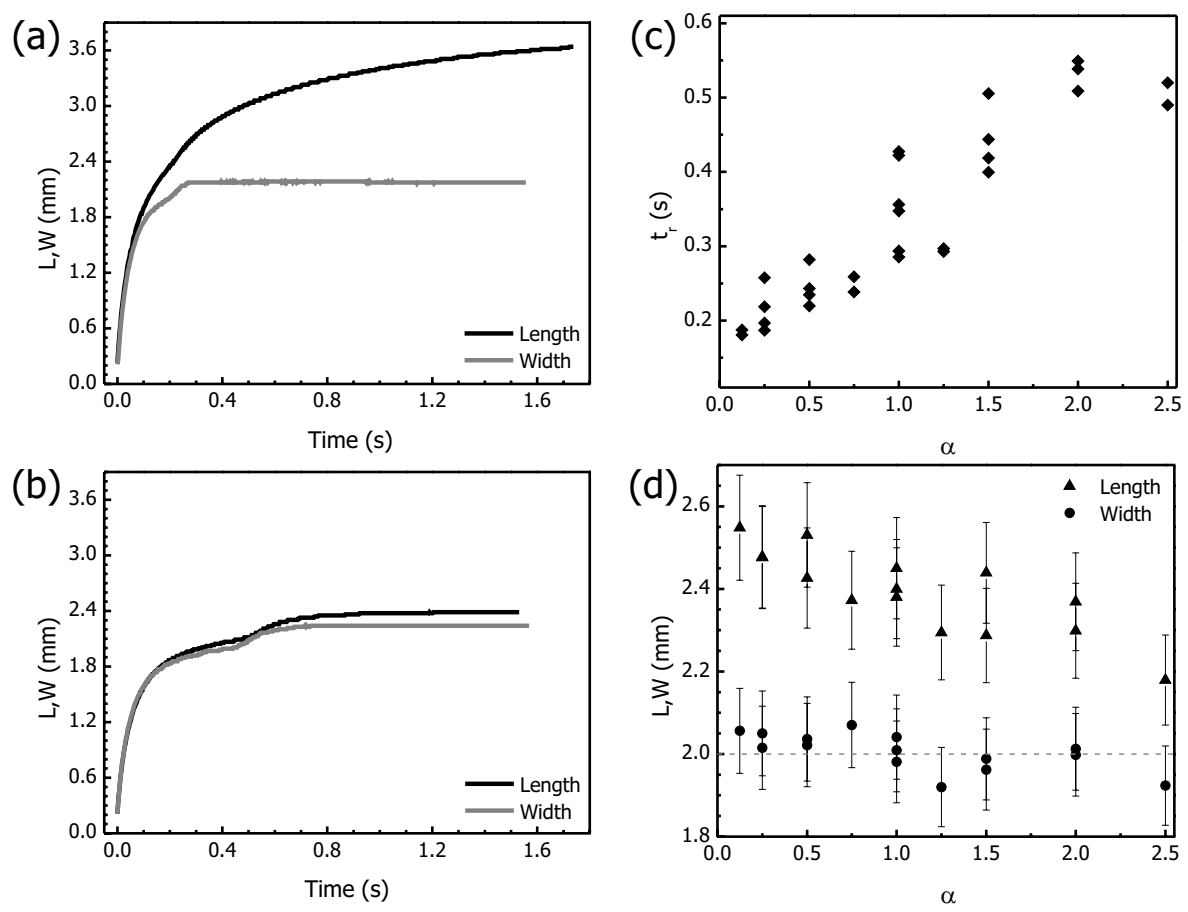

Figure 3.3: Evolution of the base of the droplet as a function of time for two patterns with (a) $\alpha=0.125$ (stripe widths: $16 \mu \mathrm{m} \mathrm{SiO}_{2} / 2 \mu \mathrm{m}$ PFDTS) and (b) $\alpha=2.5$ (stripe widths: $8 \mu \mathrm{m} \mathrm{SiO}_{2} / 20 \mu \mathrm{m}$ PFDTS); only the first $1.8 \mathrm{~s}$ are shown as most of the dynamics takes place in this time frame. In case of (a) the final shape is reached after 10s, while for (b) the final shape is achieved within the studied timeframe. (c) The time of release $t_{\mathrm{r}}$ of a droplet from the needle is plotted as a function of $\alpha$. (d) $L\left(t_{\mathrm{r}}\right)$ and $W\left(t_{\mathrm{r}}\right)$ at the moment the droplet releases from the needle plotted as a function of $\alpha$. The values of $W\left(t_{\mathrm{r}}\right)$ scatter around a mean value of $2 \mathrm{~mm}$ (dotted line) for all $\alpha$ values, while the $L\left(t_{\mathrm{r}}\right)$ values exhibit a decline as the hydrophobicity of the substrate increases.

\subsubsection{Spreading kinetics dependence on surface chemistry}

The dependence of spreading kinetics on relative hydrophobicity of the underlying pattern can be best illustrated by considering the time span between the moment of contact with the surface and detachment from the needle, which we designate $t_{\mathrm{r}}$ (Fig. 3.3 (c)). The plot reveals that the $t_{\mathrm{r}}$ values increase as a function of $\alpha$. The reason for $t_{\mathrm{r}}$ increase is given in Fig. $3.3(\mathrm{~d})$ where the values of the width and the length at $t_{\mathrm{r}}, W\left(t_{\mathrm{r}}\right)$ and $L\left(t_{\mathrm{r}}\right)$ respectively, are plotted. 


\subsection{RESULTS}

The values of $W\left(t_{\mathrm{r}}\right)$ at the moment the neck breaks off scatter around approximately $2 \mathrm{~mm}$ (dashed line in Fig. $3.3(\mathrm{~d})$ ) while the length at $t_{\mathrm{r}}$ is not constant. Assuming that reaching a certain $W\left(t_{\mathrm{r}}\right)$ value is the necessary condition for release from the needle to occur, the trend of $t_{\mathrm{r}}$ can be explained as follows. Advancing across a PFDTS stripe represents a barrier for spreading as energy is lost on depinning of the contact line at the chemical border of the stripe and the unfavourable wetting of a hydrophobic surface. In contrast, the hydrophilic stripe is wetted immediately once the contact line gets in contact with it. Consequently, the larger is the proportion of hydrophilic $\mathrm{SiO}_{2}$ stripes in the pattern or the lower the $\alpha$ is, the faster the required width will be reached. Summarizing, $t_{\mathrm{r}}$ is a good parameter for comparison of spreading kinetics on different patterns as: (i) reaching certain diameter $W\left(t_{\mathrm{r}}\right)$ length defined by properties of hydrophobic part of the stripe pattern is the trigger event for the release to occur; (ii) the hydrophilic part of the pattern defines the timespan for this critical $W\left(t_{\mathrm{r}}\right)$ to be reached.

Once the release event has occurred, the jump in evolution of $L(t)$ and $W(t)$ (Fig. $3.3(\mathrm{a}, \mathrm{b}))$, or temporally enhanced spreading, takes place. The reason for the observed behaviour can be understood by making a comparison of a droplet with a spring of stiffness $\gamma$ that is released at a time $t_{\mathrm{r}}$ liberating the stored potential energy, as suggested by Biance et al. [53]. While the droplet is attached to the needle, there are two forces that compete: (i) the interaction between needle and glycerol, arising from capillary effects, that pull the droplet up and (ii) the interaction between the glycerol and the surface that induces spreading and pull the droplet down. Once the neck connecting the droplet to the needle breaks, the center of mass of a droplet shifts downwards, inducing a sharp increase of the CAs. Increase in the CAs will in turn make the contact line advance at a higher pace until the value preceding the release event will be reached, which results in enhanced spreading as observed in the graphs showing the evolution of the diameters. For all $\alpha$ studied, the jump is more pronounced for $W(t)$ as compared to $L(t)$, and is absent in the length evolution $L(t)$ in case of $\alpha<1$.

To account for the fact that the jump occurs in the evolution of $W(t)$ for all $\alpha$, but only for $\alpha>1$ in case of $L(t)$, the plot of $W\left(t_{\mathrm{r}}\right)$ and $L\left(t_{\mathrm{r}}\right)$ in Fig. 3.3 (d) can be used. In the case of $\alpha>1$, the values of $L\left(t_{\mathrm{r}}\right)$ are close to those of $W\left(t_{\mathrm{r}}\right)$, the $\theta_{\|}(t)>90^{\circ}$, and the jump is observed. In case of $\alpha<1$, elongation in the direction of the stripes is present, giving slightly larger $L\left(t_{\mathrm{r}}\right)$ values as compared to $W\left(t_{\mathrm{r}}\right)$, but $\theta_{\|}(t)<90^{\circ}$ (Fig. $3.2(\mathrm{~g})$ ): the jump is not observed. The results suggest that the absolute distance from the needle to the contact line and the CA values are the key parameters for the explanation of the 
occurrence of the jump.

Finally, comparing $W\left(t_{\mathrm{r}}\right)$ and $L\left(t_{\mathrm{r}}\right)$ with static length and width values reveals that the detachment from the needle takes place once $90 \%$ of the final width $W_{\mathrm{St}}=2.15 \mathrm{~mm}$ for all $\alpha$ and between $65 \%$ and $90 \%$ of the final length $L_{\mathrm{St}}$ is reached. Moreover, shortly after the release event has taken place, $W(t)$ reaches its final value and $L(t)$ continues to grow at a speed of less than $1 \mathrm{~mm} / \mathrm{s}$ until the final equilibrium shape of a droplet is reached. From the result shown in Fig. 3.3 (d) it appears that the strong elongation in the direction parallel to the stripes, observed for small $\alpha$, will only occur in a later stage of spreading, and is not very pronounced in the time lapse prior to detachment.

\subsubsection{First stage of spreading}

As described in the previous section, the droplet adopts up to $90 \%$ of its final shape in less than $0.5 \mathrm{~s}$. Consequently, a more thorough study of the very first stage of spreading, which takes place immediately after the contact with the surface, is required to fully understand the final equilibrium shape of the droplets

In Fig. 3.4 the length $L(t)$ and width $W(t)$ for two values of $\alpha$ are shown for the time span between touching of the surface up to the moment of detaching from the needle. These graphs demonstrate that in the beginning of spreading both $L(t)$ and $W(t)$ evolve at the same rate; only after approximately $50 \mathrm{~ms}$ the spreading in the direction parallel to the stripes becomes markedly faster than in the perpendicular direction. Similar isotropic initial spreading behaviour, independent of the surface structure, was also observed for the droplets jetted on grooved hydrophobic surfaces [54]. Kannan et al. [54] attributed the circular area observed in the initial spreading regime to the inertia of the moving droplet in the kinematic stage of deposition (velocities of droplets $>1 \mathrm{~m} / \mathrm{s}$ ). In our case the impact velocity is considerably smaller, i.e. $<5 \mathrm{~mm} / \mathrm{s}$. For such low velocities the influence of the kinetic energy is not expected to play a dominant role in the motion of the contact line, as pointed out by Biance et al. [53] and only surface-liquid interactions are responsible for spreading.

The fact that we observe isotropic growth of the droplet base in the first stage of spreading implies that the evolution of the wetted area in this time interval is not sensitive to the specific pattern on the surface. As can be concluded from the log-log plots in Fig. 3.4, the transition from isotropic (circular shape) to anisotropic (elongated shape) spreading, owing to a preferential spreading in the direction along the stripes, occurs after ap- 


\subsection{RESULTS}
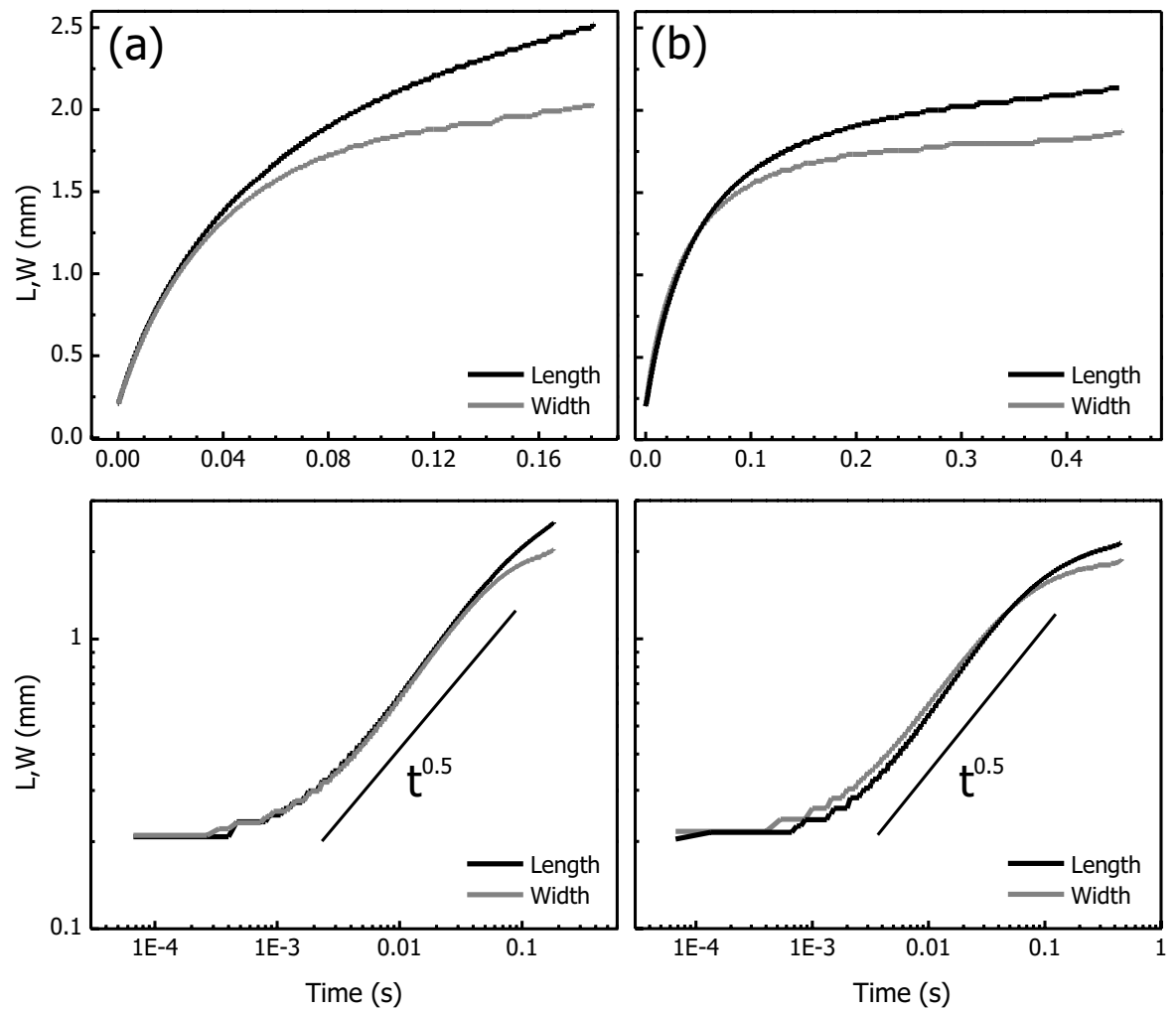

Figure 3.4: Time evolution of length $L(t)$ and width $W(t)$ plotted for (a) $\alpha=0.125$ and (b) $\alpha=2.5$, up to the moment of release from the needle, at (a) $0.18 \mathrm{~s}$, and (b) $0.50 \mathrm{~s}$, respectively. The bottom row of figures represents log-log plots of the same data presented in the top row, to enable identification of a power-law behaviour in spreading of the $L(t)$ and $W(t)$. The solid lines with a slope of 0.5 , serving as a guide to the eye, correspond to the inertial spreading behaviour, as discussed further in the text.

proximately $50 \mathrm{~ms}$ for all $\alpha$. It can be concluded that the initial spreading is only weakly influenced by the substrate properties, and seems to be defined by the liquid itself. 


\subsection{Discussion}

\subsubsection{State of art}

In the thermodynamical approach, theoretical and practical studies of spreading of liquid on dry surfaces in case of complete wetting have shown: (i) the time evolution of the diameter of the contact area can be correlated by a power law as a function of time $D=K t^{n}$, where $K$ and $n$ are constants; (ii) two regimes of spreading, having characteristic coefficients $n$, are observed [41, 55-57]. The nature of the forces that drive and oppose the motion of the contact line define the regime the droplet is in and determine the coefficient $n$ of the power law. In the first stage of spreading, on short time scales, viscous forces oppose the capillary driving forces. The evolution of the droplet base radius follows a power law, commonly referred to as the Tanner law [58], giving $r(t) \propto t^{1 / 10}$. Experimentally observed evolution of the droplet base radii for various liquids follow the theoretically calculated 0.1 exponent [59]. This regime is often referred to as the capillary or viscous stage. Once the dimension of the droplet exceeds the capillary length, gravitational forces start playing a dominant role and the radius scales as $t^{1 / 8}[60]$.

In the case of partial wetting, scaling with time following a power law is also observed, though the coefficients are found to be higher than in the case of complete wetting. The experimental values depend on the system under consideration, though the correlation of particular properties like surface tension, contact angle, etc. and measured coefficients is yet to be elucidated [56, 57].

However, in case of a thermodynamical approach, a problem arises when attempting to model the initial stage of spreading, taking place immediately after the contact with the substrate. The calculated velocity of the contact line diverges, what is known as Huh and Scriven paradox [61]. A solution was proposed by Biance et al. [53], based on experimental studies on completely wetting glass surface using a high speed camera. They identified a new regime, which they designated as the inertial regime, which precedes the capillary regime. On the basis of their results, they proposed a model derived from that adopted by Eggers et al. [62] for the initial moments of coalescence of two droplets. Biance et al. [53] suggested that the gradient of the curvature at the contact line between solid and liquid acts as the driving force for spreading in this regime. The inertia of the liquid at the center of mass of droplet is assumed to be the limiting factor for spreading. From this inertial model, $r(t)$ should scale with $t^{0.5}$, which was indeed confirmed by experimental results [53, 56, 63]. Additionally, 


\subsection{DISCUSSION}

the transition to the capillary regime, governed by $r(t) \propto t^{1 / 10}$ was clearly identified $[53,63]$. The duration of inertial regime, estimated in the frame of the model proposed by Biance et al. [53], is found to be of the order of a few $\mathrm{ms}$ and decreases as the liquid viscosity increases. Simulations carried out on the basis of the proposed inertial model show good agreement with the experimental results [64].

The aforementioned work pertained to homogeneous, uniform completely wetting surfaces. In the case of partial wetting, only a few experimental studies of the inertial regime of spreading have been described [56, 63]. The results show that the exponent of the power law is not unique and depends on the surface energy: a net decrease from 0.5 for hydrophilic surfaces to 0.3 on hydrophobic surfaces was observed. Following the inability of the model by Biance et al. [53] to account for the variations of the exponent with the contact angle, Bird et al. [63] proposed an alternative approach, in which the motion of the liquid close to the surface is responsible for spreading.

\subsubsection{Inertial stage of spreading}

Log-log plots allow direct estimation if the power-law growth for the spreading on a surface is followed. If so, the exponent can be determined directly, given by the slope of the straight line fitted to the part where the data exhibits linear behaviour. In the log-log plots of Fig. 3.4 two regimes can be identified. Prior to the spreading of the droplet, the diameter of the wetted area amounts to $200 \mu \mathrm{m}$ for all $\alpha$ considered in this work. The increase of the diameter in the first regime indeed seems to follow a power law-like growth with an exponent close to 0.5 , corresponding to the one expected in case of inertial spreading, i.e. $L(t) \approx W(t) \propto t^{0.5}$. In the subsequent regime, after approximately $50-100 \mathrm{~ms}$, the slopes of the log-log plots become markedly smaller than 0.5 and exhibit a clear dependence on the direction of spreading and underlying surface chemistry. The time-scale for the second regime is relatively short as a result of the droplet detachment from the needle. A power-law can not be identified; in the analysis of our results we only refer to the slope of the transients in this regime.

As the transition between the two regimes is smoothened most probably due to presence of two chemical species with different wettabilities on the surface, in order to make a proper fit of experimental data, a measurable parameter has to be defined that will serve as transition criteria. In the publication by Bird et al. [63], they observed that the transition on the hydrophobic surface occurred once the spreading radius reached 0.9 of the 
initial drop radius, i.e. at a time $t_{0.9}$. To check the validity of this criterion, in Fig. 3.5 (a) we plot $t_{0.9}$ values for all $\alpha$ studied. From the plot it follows that the determined $t_{0.9}$ values increase as the hydrophobicity of surface increases, changing from $50 \mathrm{~ms}$ in case of small $\alpha$ to close to $90 \mathrm{~ms}$ for large $\alpha$. From the data presented in Fig. 3.4 one can see that for $\alpha$ of 2.5 the change of the slope effectively takes place somewhat later than for $\alpha=0.125$. The scattering of data can be a result of a different absolute width of PFDTS and $\mathrm{SiO}_{2}$ stripes that would correspond to the same $\alpha$. Using retrieved values for $t_{0.9}$ to fit the transition of both regimes appears to give an excellent agreement with the experimental data.

In Fig. 3.5 (b) the experimental spreading coefficients $n$ according to $D \propto$ $t^{n}$ for $L(t)$ and $W(t)$ in the inertial regime are plotted as a function of $\alpha$. The values of $n$ seem to depend on the relative hydrophobicity of the surface, exhibiting a slight decrease from the values observed on superhydrophilic surfaces $(n=0.5)$, to the values for unpatterned PFDTS of 0.43 . Nevertheless, both $L(t)$ and $W(t)$ evolve at the same rate, exhibiting direction dependence only in the subsequent capillary regime. This implies that in the inertial regime, the precise spatial distribution of the patterned functionalized surface is not important, and exponents depend only on the relative hydrophobicity of the surface expressed in terms of $\alpha$.

Interestingly, the inertial regime in our measurements lasts considerably longer than what was predicted in the model or observed for water droplets. The reproducibility of this observation has been thoroughly verified in different extensive, independent data acquisition series. Such durations cannot be explained by the propagation of a capillary wave, as described by Bird et al. [63]. It may be attributed to the considerably higher viscosity of glycerol $(\approx 1000$ times that of water), but to verify this suggestion experiments using different liquids will be performed; this will be the subject of future work.

\subsubsection{Capillary stage of spreading}

Once the transition to the capillary regime has taken place, interactions between liquid and surface drive spreading, inducing different growth of orthogonal diameters $L(t)$ and $W(t)$. As the detachment event induces a jump in the evolution of the diameter during this stage, the duration is not long enough to assess any power law behaviour. Nevertheless, in order to discuss spreading properties and to investigate its dependence on the relative hydrophobicity of the surface, the data between $t_{0.9}$ and the moment the droplet detaches from the needle is fitted to retrieve an estimated slope. 


\subsection{DISCUSSION}
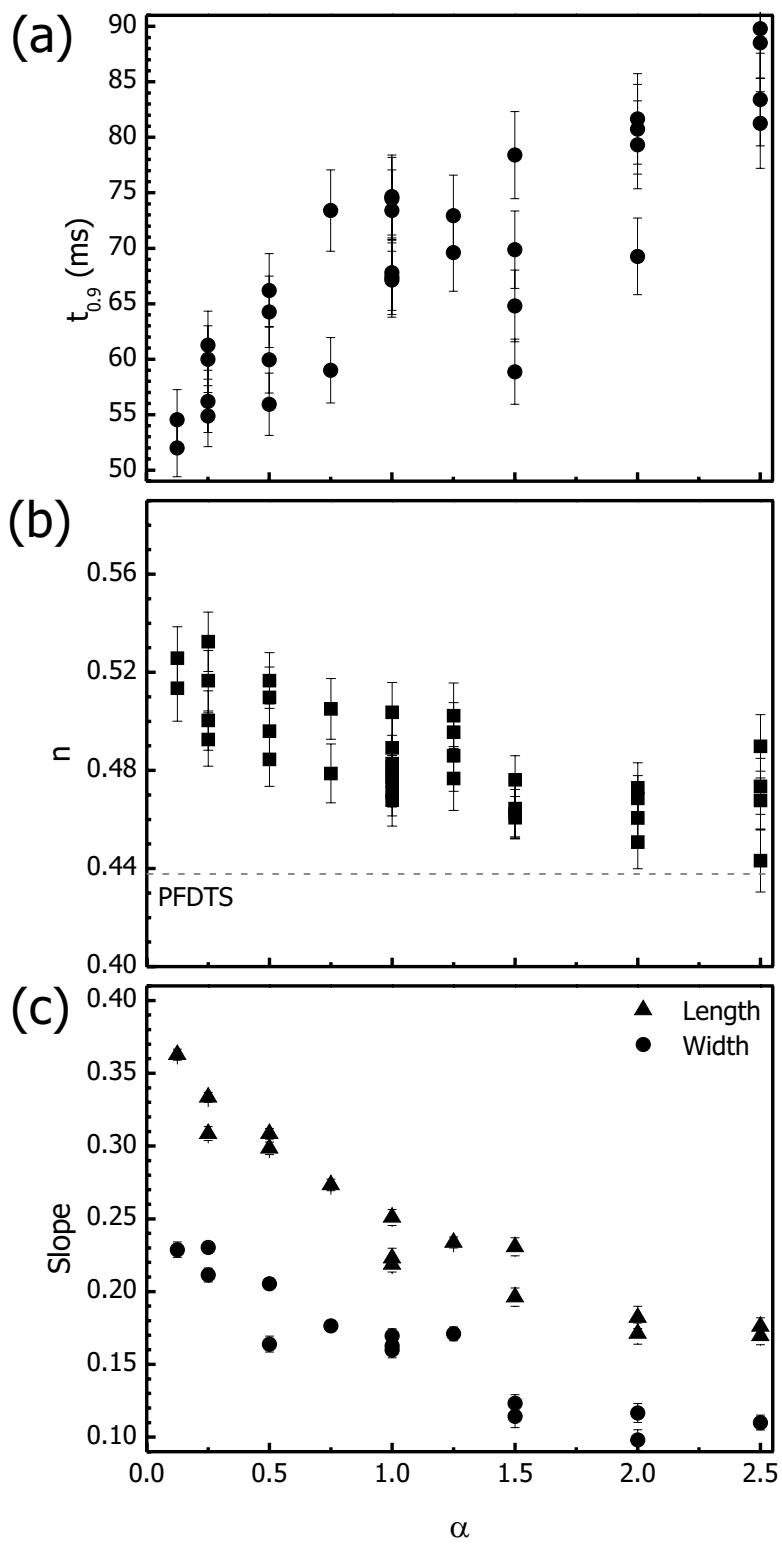

Figure 3.5: (a) Experimentally determined time $t_{0.9}$ at which the spreading radius amounts to $90 \%$ of the radius of suspended droplet prior to deposition, plotted as function of $\alpha$. The values exhibit a linar increase from the value for pristine $\mathrm{SiO}_{2}$ (50ms) to the value for unpatterned PFDTS $(99 \mathrm{~ms})$. The error bars correspond to an average error of $5 \%$. (b) Power-law coefficients $n$ for the inertial regime obtained from the experimental data using $t_{0.9}$ as the transition criteria plotted as a function of $\alpha$. (c) Slopes estimated for the spreading in the capillary regime plotted as a function of $\alpha$. The error-bars reflect only the standard deviation from fitting the experimental data. 
In Fig. 3.5 (c) the experimental slopes for $W(t)$ and $L(t) \log$-log plots are given for all $\alpha$ values studied. The slope values differ depending on the direction of spreading due to a well-defined pattern. For both $L(t)$ and $W(t)$ the values have a tendency to decrease for larger $\alpha$, although significant scattering is observed. In case of the width $W(t)$, the slopes decrease from 0.2 to 0.1 , the latter one being the value expected in case of spreading on completely wetting surfaces. The values for the length $L(t)$ change from 0.35 for $\alpha=0.125$ to approximately 0.15 for $\alpha=2.5$.

The fact that we observe smaller slope values in the case of spreading perpendicular to the stripes can be explained by the way the contact line advances. The mechanism for advancing of the droplet is similar to the one described earlier for grooved surfaces [29,41] and is schematically presented in Fig. 3.6. First the droplet forms a nucleus that bridges the hydrophobic PFDTS stripe and the next hydrophilic $\mathrm{SiO}_{2}$ stripe, stopping at the chemical border of the next hydrophobic stripe. Subsequently, the contact line starts to spread laterally to cover both stripes until the advancing spherical caps in the direction parallel to the stripes are reached. Simultaneous with this 'filling' of the stripes, a new nucleus may be formed that covers the next period of surface pattern.

The aforementioned mechanism is responsible for higher contact angles than in the direction parallel to the stripes. Formation of the nucleus over the stripes presents an energetic barrier for the advancing, as locally the interface has to be strongly distorted. The value of the energy barrier for nucleation should be approximately the same for all $\alpha$ studied. For increasing widths of the hydrophobic stripes the time required for its bridging will be affected; we assume this to be responsible for the decrease of the $W(t)$ evolution rate with increasing $\alpha$ or width of PFDTS stripes, as well as scattering of data for similar $\alpha$.

On the other hand, for advancing parallel to the stripes, there is a much less pronounced energy barrier for spreading [28, 39, 42, 65]. Moreover, the energy barrier is not constant for different patterns: the larger the hydrophilic $\mathrm{SiO}_{2}$ fraction on the surface, the more it is energetically favourable for the droplet to wet the surface and the faster the contact line will advance. That may be the reason for the experimentally observed decrease of the evolution rate as a function of $\alpha$ (see Fig. 3.5). For $\alpha=2.5$ its value is very close to the one in the direction perpendicular to the stripes.

Furthermore, once the transition from the inertial to the capillary regime occurs, the center of mass of the droplet is higher than its equilibrium position; it will continue to move downwards. However, the excess liquid coming from this downshift cannot be easily accommodated by increasing 


\subsection{CONCLUSIONS}

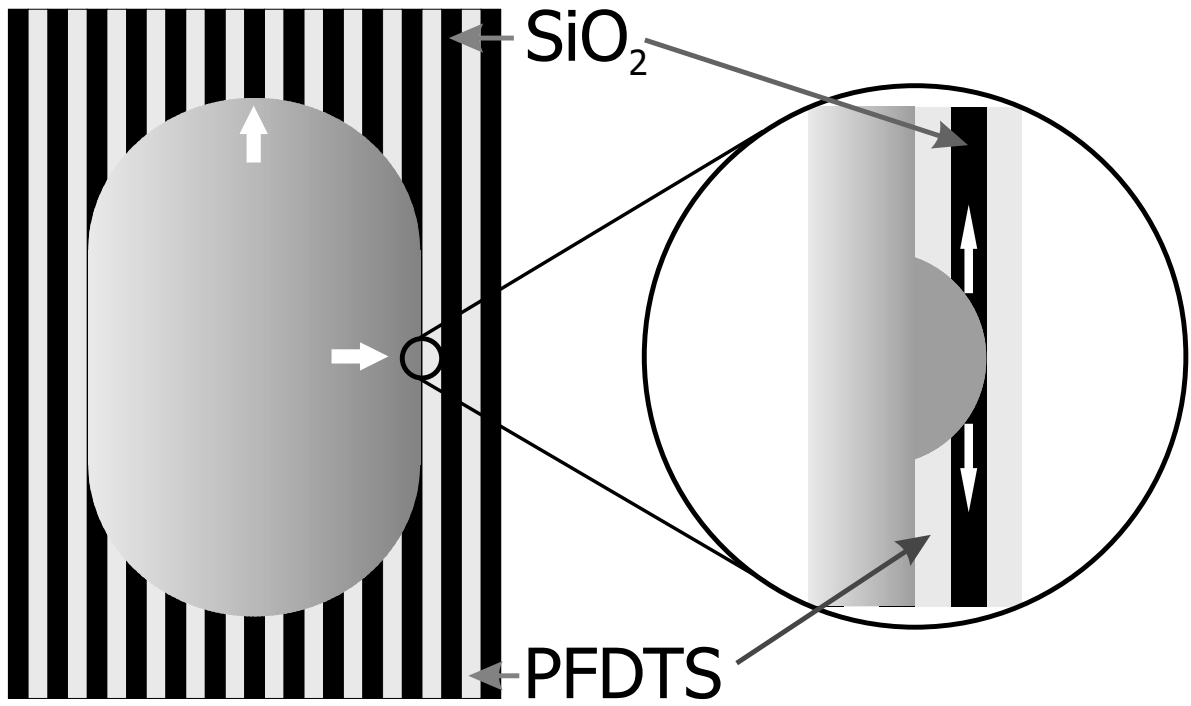

Figure 3.6: (Schematic representation of the way in which the droplet advances in the direction perpendicular to the stripes. Bulging of the small volume of the liquid in the form of nucleus that covers the upcoming PFDTS and $\mathrm{SiO}_{2}$ stripes is shown. This acts as an energy barrier for spreading. Once the nucleus is formed, the liquid fills the two stripes in both directions until the new edge of the advancing spherical cap is formed.

the spreading rate in the direction perpendicular to the stripes. In addition, the pattern under the droplet facilitates the flow near the surface in the direction parallel to the stripes, along the hydrophilic $\mathrm{SiO}_{2}$ stripes, therewith creating stream lines. Consequently, the liquid is 'pushed' in the direction parallel to the stripes by the combined effect of the lateral confinement due to the energy barrier in the orthogonal direction and stream lines due to the surface pattern. Such a 'squeezing' mechanism may well account for the higher slope in the length evolution of $L(t)$ that we observed for small $\alpha$.

\subsection{Conclusions}

Summarizing, we have performed a fast camera study of the kinetics of spreading of glycerol droplets on the chemically patterned surfaces. Plotting the evolution of two diameters $L(t)$ and $W(t)$ as a function of time shows that up to $90 \%$ of the dynamics takes place in the first $0.5 \mathrm{~s}$. A more 
detailed study of the first instances of spreading reveals that two regimes of spreading are observed. The first regime is identified as inertial, which is followed by the capillary regime. The duration of the inertial regime is measured to be longer than what is theoretically predicted, which may be attributed to the high viscosity of the glycerol. In the capillary regime, experimentally measured evolution rates differ for the orthogonal spreading directions, with higher values in the direction parallel to the stripes; we explain this by a 'squeezing' of the liquid by the hydrophobic part of the pattern. 




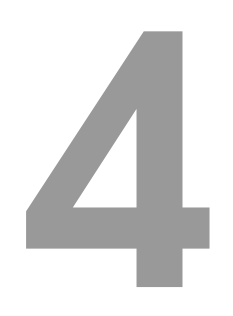

\section{Smart design of stripe-patterned gradient surfaces to control droplet motion}

The motion of droplets under the influence of lithographically created anisotropic chemically defined patterns is described and discussed. The patterns employed in our experiments consist of stripes of alternating wettability: hydrophobic stripes are created via fluorinated self-assembled monolayers (SAMs), and for hydrophilic stripes the $\mathrm{SiO}_{2}$ substrate is used. The energy gradient required to induce motion of the droplets is created by varying the relative widths of the stripes in such a way that the fraction of hydrophilic matter increases. The anisotropic patterns create a preferential direction for liquid spreading parallel to the stripes and confine motion in the perpendicular direction, giving rise to markedly higher velocities as compared to non-structured surface energy gradients. Consequently, the influence of the distinct pattern features on the overall motion as well as suggestions for the design improvements from an application point of view are discussed. 


\subsection{Introduction}

Liquid motion on solid surfaces without any externally applied force was already observed a long time ago: the "tears" of wine have been used for centuries to judge its quality [66]. Nevertheless, it was not before the second half of the $19^{\text {th }}$ century that variation of the liquid-vapour surface energy $\gamma_{1 v}$ was identified as the driving force for wine rising on the glass wall: a phenomenon nowadays referred to as Marangoni flows [67, 68]. Other examples of droplet motion resulting from Marangoni forces include thermal gradient induced movement [69-71], originating from the fact that the surface tension is temperature dependent, forcing droplets to colder areas, and also surfactant induced motion [72], in which liquid-vapor surface tension gradients arise from varying concentration of molecules at the liquid surface. The first models to predict droplet velocities were reported in the end of the previous century $[73,74]$. Due to complexity of the problem, models are still to be refined, leaving many questions concerning Marangoni flows to be elucidated [75].

Using a surface tension gradient of the solid surface to induce motion avoids Marangoni flows [69]. A surface energy gradient as schematically shown in Fig. 4.1, is typically achieved by controlled chemical modifications of the substrate. The surface tension increases in a specific direction, changing from hydrophobic $\left(\gamma_{\mathrm{sv} 1}\right)$ to hydrophilic $\left(\gamma_{\mathrm{sv} 2}\right)$. Owing to the different surface energy, the difference in dynamic contact angles (CAs) will induce movement in the direction of increasing $\gamma_{\mathrm{sv}}$ [76-79]. Experimental motion studies reveal the velocities to depend linearly on the radius of the wetted area; moreover the droplet will move to the hydrophilic region only when the radius is above a certain critical value [80,81].

In this chapter, we present a detailed investigation into the motion of

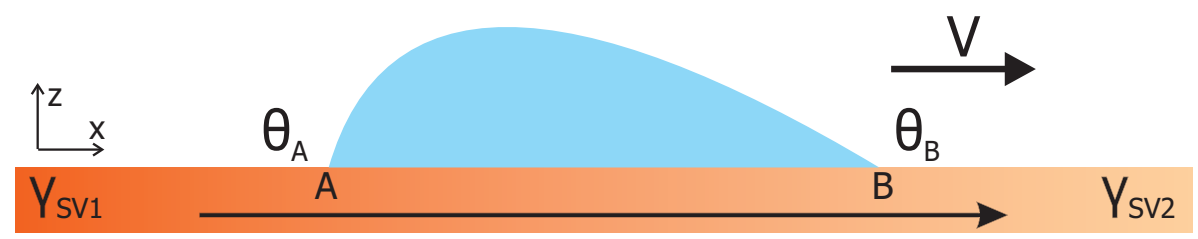

Surface tension gradient

Figure 4.1: Schematic representation of a sessile droplet crossection on a wettability gradient changing from hydrophobic (low $\left.\gamma_{\mathrm{sv} 1}\right)$ to hydrophilic $\left(\right.$ high $\left.\gamma_{\mathrm{sv} 2}\right)$, i.e. $\gamma_{\mathrm{sv} 2}>\gamma_{\mathrm{sv} 1}$. The droplet will move in the direction of high $\gamma_{\mathrm{sv} 2}$ with a speed $V$. 


\subsection{THEORY}

droplets on chemically patterned surfaces. The droplets are gently deposited on a well-defined pattern, a driving force being generated by a macroscopic wettability gradient. The overall motion of droplets under the influence of the surface energy gradient is described. The different regimes in the droplet motion are identified and discussed. Finally, the effect of the underlying pattern in relation to liquid motion is discussed, with a view to possible applications.

\subsection{Theory}

Summarizing existing descriptions, the droplet velocity is determined by the balance of driving capillary and opposing viscous forces [80, 82]. The driving force of motion is the unbalanced Young force $F_{\mathrm{Y}}$ originating from different surface tensions on both sides of the droplet:

$$
\mathrm{d} F_{\mathrm{Y}}=\left[\left(\gamma_{\mathrm{sv}}-\gamma_{\mathrm{sl}}\right)_{\mathrm{B}}-\left(\gamma_{\mathrm{sv}}-\gamma_{\mathrm{sl}}\right)_{\mathrm{A}}\right] \mathrm{d} x
$$

The contact line at points $\mathrm{A}$ and $\mathrm{B}$ probes two different surface surface energies $\left(\gamma_{\mathrm{SV}}\right)_{\mathrm{B}}>\left(\gamma_{\mathrm{SV}}\right)_{\mathrm{A}}$ and consequently adopts different contact angles $\theta_{\mathrm{B}}<\theta_{\mathrm{A}}$. These local dynamic contact angles can be used to rewrite the eq. 4.1:

$$
\mathrm{d} F_{\mathrm{Y}}=\gamma_{\mathrm{sv}}\left(\cos \theta_{\mathrm{B}}-\cos \theta_{\mathrm{A}}\right) \mathrm{d} x
$$

This equation expresses the fact that ideally as soon as the contact angle $\theta_{\mathrm{B}}$ becomes smaller than $\theta_{\mathrm{A}}$, the droplet experiences a driving force and will start to move in the direction of the higher $\gamma_{\mathrm{sv} 2}$.

The motion of the droplet is hindered by viscous drag, also referred to as the friction force $F_{\eta}$. Using the lubrication approximation and assuming a circular shape of the wetted area (radius $R$ ) it is given by [74]:

$$
F_{\eta}=3 \eta \pi R V \int_{x_{\min }}^{x_{\max }} \frac{\mathrm{d} x}{\xi(x)}
$$

where $\eta$ is the viscosity of the liquid, $V$ is the droplet velocity, and $\xi(x)$ is the height of the droplet. The integration limits $x_{\min }$ and $x_{\max }$ are two cutoff lengths, the first being of molecular dimensions and the second is of the order of $R$.

If only these two forces are considered, the estimated viscous drag is orders of magnitude smaller than the driving force [83]. As such, droplets are expected to move on all surfaces as soon as the CAs on both sides are different. Nevertheless, experimental results reveal that in many cases droplets remain immobile despite the CA difference (eq. 4.2). 
The explanation is provided by the oversimplified initial model, in which surfaces without hysteresis are considered. Only on ideal surfaces, eq. 4.2 and eq. 4.3 describe the droplet velocity. In general, experimental surfaces exhibit hysteresis and as such provide a supplementary energy barrier for droplet motion [76, 81, 84]. The hysteretic force can be described in terms of the advancing and receding angles on opposing sides of the droplet:

$$
\mathrm{d} F_{\text {hys }}=\gamma_{\text {Iv }} \int\left(\cos \theta_{\mathrm{Ad}, \mathrm{B}}-\cos \theta_{\mathrm{Re}, \mathrm{A}}\right) \mathrm{d} x
$$

Combining the driving force (eq. 4.2) with the two resisting forces (eq. 4.3 and eq. 4.4) provides a general description of the velocity on most experimental surfaces.

The aforementioned approach holds for free standing droplets. However, in experimental situations, droplets are either (i) gently deposited while remaining attached to a dispensing unit or (ii) dropped from finite heights. In the latter case, the impact event itself should be considered also as it introduces kinetic energy into the system, which interferes with the droplet motion. On the other hand, during controlled gentle deposition liquid-surface interactions govern the motion of the droplet. Nevertheless, in the initial stages of motion the surface energy gradient induces a force on the droplet, while it remains attached to the needle. The capillary force gives rise to an additional force that has to be taken into account. In many literature reports, this influence is overlooked, and droplets are generally considered as free standing.

\subsection{Experimental details}

\subsubsection{Chemically created pattern}

The surface patterns of self-assembled monolayers (SAMs) of $1 \mathrm{H}, 1 \mathrm{H}, 2 \mathrm{H}, 2 \mathrm{H}-$ perfluorodecyltrichlorosilane (PFDTS, 97\%, ABCR, Germany) on silicon wafers are created using standard clean room facilities. First, positive photoresist is spin-coated on freshly cleaned wafers with a natural oxide film, followed by soft-baking. Patterns are created via standard optical lithography, after which the exposed photoresist is washed-off. The remaining photoresist is hard-baked and will provide surface protection during vapour deposition of PFDTS; the silane head-group binds covalently to the native silicon oxide, exposing the fluorinated tail to the liquid. The assembly creates a densely packed layer of molecules with a height in the order of one $\mathrm{nm}$. Vapour deposition is done in a degassed chamber that 


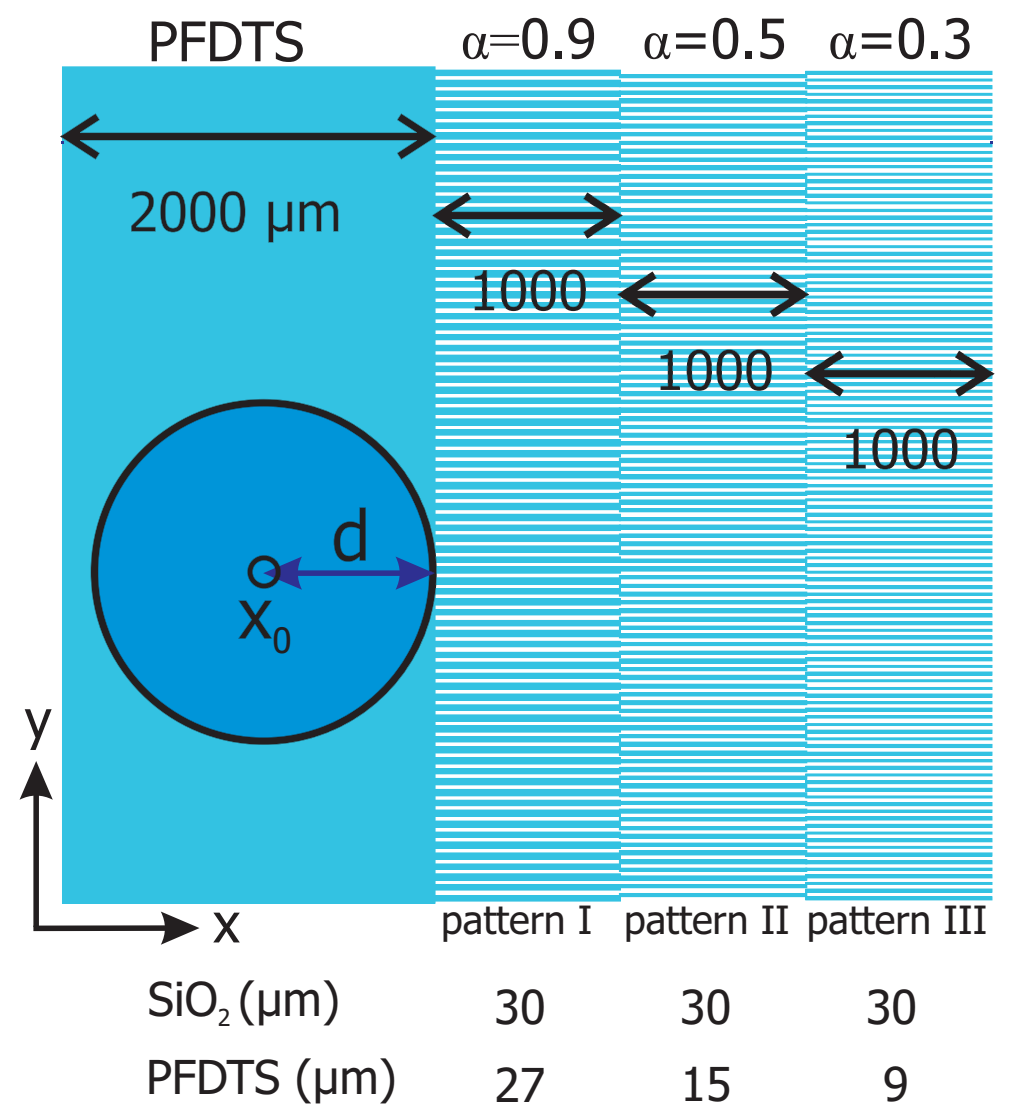

Figure 4.2: An example of the gradient pattern design on a silicon wafer. Hydrophobic regions (blue) are created by PFDTS SAMs, while bare $\mathrm{SiO}_{2}$ (white) comprises the hydrophilic areas. The dark blue circle depicts the droplet contact area during the initial spreading regime. The point $x_{0}$ defines the center of the wetted area, i.e. where the droplet contacts the surface. The distance $d$ between the point of contact and the border of the first patterned area is determined during movie analysis. Below the respective patterns, the absolute widths of the stripes are indicated (in $\mu \mathrm{m}$ ) which are used for $\alpha$ calculation (eq. 4.5). This particular pattern is used for the experimental data presented in the side- and top- view movies in the next section.

is successively exposed to PFDTS and water reservoirs to introduce the respective vapours, initiating the reaction on the wafer surface [34]. After SAM formation, the photoresist is washed off, leaving a chemically patterned surface.

A typical layout of the patterns used in our experiments is shown in 
Fig. 4.2. The droplet is deposited on the rectangular area of unpatterned PFDTS having a width of $2 \mathrm{~mm}$; this is slightly larger than the $1.55 \mathrm{~mm}$ diameter of the wetted area of $2 \mu 1$ droplets used. On the right of the homogeneous PFDTS region, three regions (respectively referred to as patterns I, II and III) consisting of alternating hydrophobic (PFDTS) and hydrophilic $\left(\right.$ bare $\mathrm{SiO}_{2}$ ) stripes are defined. The length of the each individual striped patterns studied is either $1000 \mu \mathrm{m}$ or $700 \mu \mathrm{m}$. The striped patterns give rise to anisotropic wetting properties in orthogonal directions, favouring motion of the contact line parallel to the stripes, while hindering it in the perpendicular direction. The droplet translation is induced by the higher surface energies of the subsequent patterns, owing to an increasing fraction of hydrophilic area. To quantify the relative hydrophobicity of the patterns, we introduced a dimensionless parameter $\alpha[44,85]$ :

$$
\alpha=\frac{w_{\mathrm{PFDTS}}}{w_{\mathrm{SiO}_{2}}}
$$

where $w_{\text {PFDTS }}$ and $w_{\mathrm{SiO}_{2}}$ are the hydrophobic and hydrophilic stripe widths, respectively. Smaller $\alpha$-values imply a higher surface energy, i.e. a larger hydrophilicity of the patterns. All $\alpha$ values employed in our experiments are between 0.9 and 0.125 in different combinations. However, as the goal of this work is to present a general description of the motion over such anisotropic surfaces, we do not specifically discriminate between all different combinations of $\alpha$ in the presentation of the data.

\subsubsection{Droplet deposition}

Droplet deposition and characterization, including measurements of CAs, is done using an OCA 15+ apparatus (DataPhysics, Germany). The equipment enables determination of CAs with an accuracy of $0.5^{\circ}$. Droplets are created using a computer-controlled syringe. For the experiments in this chapter we use a $60 / 40 \mathrm{vol} \%$ mixture of glycerol (ReagentPlus, Sigma, USA) and water (Millipore Simplicity 185 system). The mixture has a surface tension comparable to that of water, while the viscosity is approximately 10 times higher than that of water. For all droplets the volume is fixed at $2 \mu$ l. Deposition of the droplet is achieved by very slowly lowering the syringe with the suspended droplet until it contacts the patterned surface. From the fast camera moves the velocity of approach is obtained. It is lower than $5 \mathrm{~mm} / \mathrm{s}$ to minimize the contribution of kinetic energy during the first stage of spreading. 


\subsubsection{High speed camera}

The time evolution of droplet shape and position is determined from sideview movies taken using a Photron SA3 high speed camera, which is operated by Photron Fastcam Viewer 3 software. Prior to capture of the movie, a $2 \mu 1$ glycerol/water droplet is suspended a few millimetres above the substrate. The recording is started simultaneously with the lowering motion. The frame rate is chosen such that it enables recording of the droplet motion over the entire pattern, typically $2000 \mathrm{fps}$ for most of the acquired data. Movies are analyzed using a home-built Matlab program. A typical set of snapshots pertaining to the first, second and third regimes of motion is presented in Fig. 4.3.

Additionally, a standard CCD camera is mounted above the substrate to assess the motion of the droplets. The frame rate is fixed at $30 \mathrm{fps}$. The movies from the top-view camera are taken simultaneously with the sideview movies enabling a better visualisation of the motion process.

\subsection{Results}

\subsubsection{Motion overview}

In Fig. 4.4, top view images of the motion of a droplet over the patterned surfaces provide a general overview. The droplet is brought into contact with the surface on the unpatterned PFDTS area on the left of the stripepatterned regions (Fig. 4.4 (a)). The location of deposition is chosen in such a way that the droplet encounters pattern I before the static shape on the PFDTS is reached. Pattern I has a higher overall surface energy due to the presence of hydrophilic $\mathrm{SiO}_{2}$ stripes, inducing a preferential direction of spreading. This in turns gives rise to an asymmetry of the droplet footprint (Fig. 4.4 (b)). Moreover, the contact line in the first striped pattern I is distorted from a circular shape.

In Fig. 4.4 (c) the droplet has detached from the needle and has moved completely off the unpatterned PFDTS rectangle onto patterns I and II. The droplet adopts an approximately cylindrical shape with two spherical caps, similar to those observed for static shapes on anisotropic patterned substrates $[42,85]$. The macroscopic surface energy of the striped patterns increases for decreasing $\alpha$, therewith inducing motion of the liquid in the positive $x$ direction. However, the droplet appears to be confined in the $y$ direction by the energy barrier created by the PFDTS stripes.

In Fig. 4.4 (d), the advancing side of the droplet has crossed pattern III 


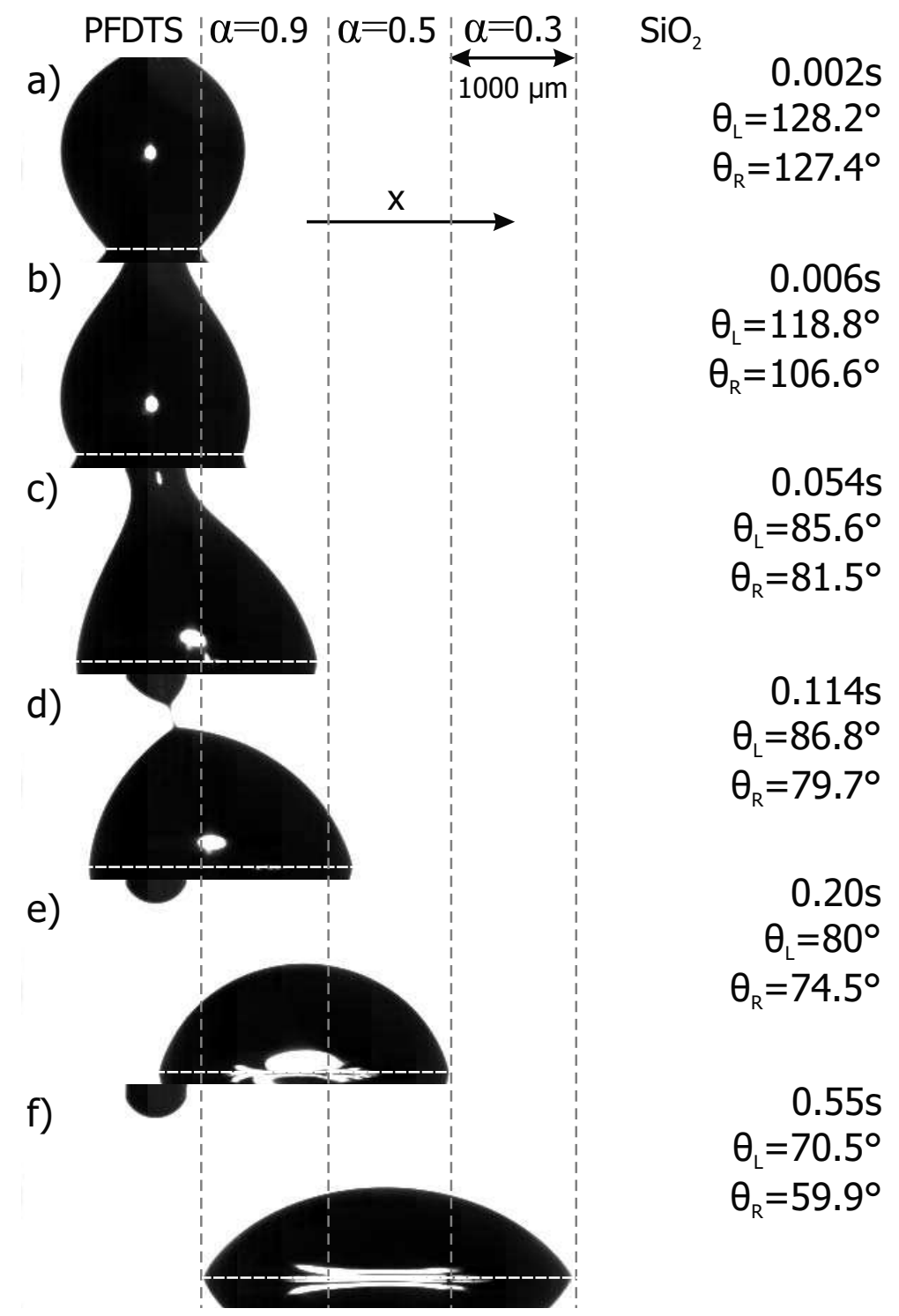

Figure 4.3: Snapshots taken from the high speed camera movies showing the side-view of droplet motion $(d=0.36 \mathrm{~mm})$. The values for dynamic CAs for the advancing (right) side: $\theta_{\mathrm{R}}$ and receding (left) side $\theta_{\mathrm{L}}$ during different regimes are presented. Vertical dashed lines indicate the borders between patterns, as introduced in Fig. 4.2 (a) The droplet has a symmetrical shape. (b) The droplet at the end of the first regime: the shape is asymmetric $\left(\Delta \mathrm{CA} \approx 9^{\circ}\right)$ due to spreading of the right side over pattern I. (c) The droplet spreading preferentially over the pattern I. (d) The end of the second regime when the "neck" breaks is shown. The asymmetry of the liquid-air interface due to neck connection is obvious. (e) The droplet during enhanced translation due to the neck breaking. (f) The droplet at the end of the third regime, covering all three striped patterns. 


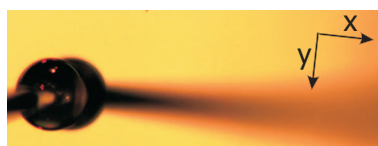

(a)

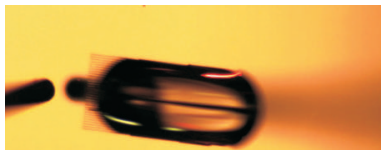

(d)

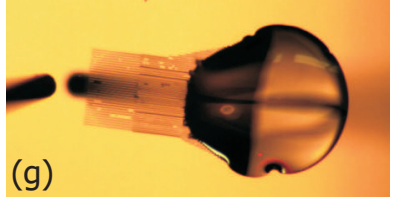

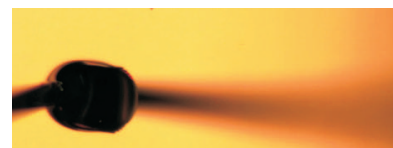

(b)
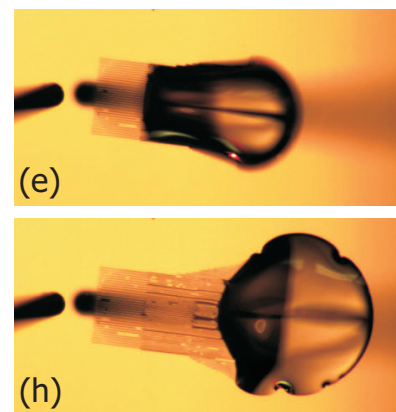

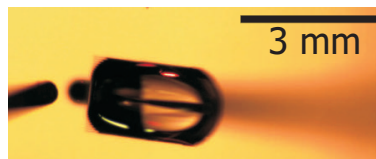

(c)

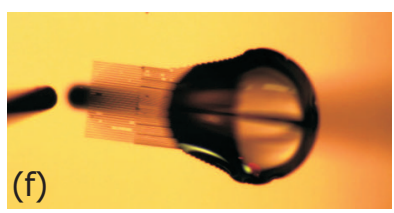

(i)

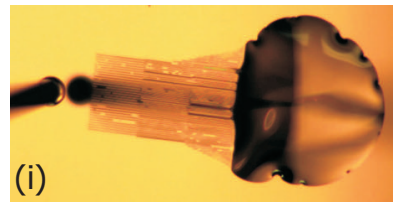

Figure 4.4: The motion of a $2 \mu 1$ glycerol/water droplet on a pattern with subsequent $\alpha$-values of $0.9,0.5$ and 0.3 . (a) The suspended droplet $(r=0.78 \mathrm{~mm})$ is brought in contact with the surface. (b) While spreading over the PFDTS rectangle it encounters the pattern I. (c) The droplet is in contact with patterns I and II, (d) followed by bridges patterns I, II and III. (e) The advancing side spreads on the unpatterned $\mathrm{SiO}_{2}$, giving rise to isotropic radial spreading. (f) Most of the droplet volume is now on the $\mathrm{SiO}_{2}$; only pattern III remains covered by liquid. (g) Receding of the droplet from pattern III, (h) to leave the patterned area and (i) finally come to a halt on the $\mathrm{SiO}_{2}$.

and starts to spread on the unpatterned $\mathrm{SiO}_{2}$. Simultaneously, the receding side starts to recede over pattern $\mathrm{I}$. On the $\mathrm{SiO}_{2}$ the droplet will spread isotropically in both $x$ and $y$ directions; the wetted area will attempt to attain a circular shape. The image in Fig. 4.4 (e) reveals that this radial spreading has an effect on the part of the droplet, which is still on the striped pattern. The energy barrier induced by the PFDTS stripes is overcome by the contact line and partial wetting of adjacent stripes on pattern III occurs. This widening continues in Fig. 4.4 (f) although not all stripes are fully wetted. Combined with the motion of the receding side over pattern II, the droplet seems to attempt to minimize its energy by adopting an approximately elliptical shape. Subsequently, the droplet continues to spread on the unpatterned $\mathrm{SiO}_{2}$ (Fig. 4.4 (g)). Eventually, the droplet slowly recedes from pattern III (Fig. 4.4 (h)) and adopts a shape close to spherical. In the last images, the effect of impurities on the $\mathrm{SiO}_{2}$ on pinning of the contact line is clearly observed.

In Fig. 4.4 (e-i) a thin residual layer of liquid can clearly be discerned 
which remains after the droplet has passed. Zooming in on the trace reveals that only the hydrophilic $\mathrm{SiO}_{2}$ stripes are covered with continuous liquid stripes,while hydrophobic PFDTS stripes remain liquid-free. However, occasionally small liquid 'bridges' covering several adjacent stripes are formed (a more detailed discussion of this residual liquid is done in Chapter 5). A quantitative studying of the liquid traces remaining after droplet passage and their influence on the receding motion is not possible in the present experimental setup and will be subject of future work. Nevertheless, the liquid trace can give us preliminary information concerning contact line motion in the direction perpendicular to the stripes. More precisely, the length diameter in the direction perpendicular to the stripes can be estimated and compared for different patterns. The preliminary results reveal that the diameter values scatter around $1.7 \pm 0.2 \mathrm{~mm}$ for all experimentally studied individual pattern lengths and combinations of $\alpha$-values. The outcome is in agreement with previously reported results for static and kinetic droplet behaviour on stripe-patterns (see Chapter 2 and Chapter 3), where we find that the length of the diameter is solely defined by the hydrophobic part of the pattern and therefore exhibits similar values for all patterns. Indeed, the diameter on unpatterned PFDTS amounts to $1.55 \mathrm{~mm}$ for the droplet volumes considered in this work, which is within the experimental error from the data extracted from top-view movies.

\subsubsection{Fast camera results}

In the previous section, movies obtained using a top view camera have been used for a qualitative description of the motion of droplets over the gradient patterned surface. However, for a quantitative study we use a high speed camera in a side-view geometry. Investigations are only carried out for the $x$ direction. A typical transient obtained using the high speed camera movies (Fig. 4.3) is shown in Fig. 4.5. The evolution of the right (advancing) and left (receding) edges of the droplet as well as the overall length of the contact area in the $x$ direction, i.e. parallel to the striped pattern is shown.

For clarity in the description, we distinguish four regimes in the droplet motion, referred to as first, second, third and fourth regimes, resp. (vertical dotted lines in Fig. 4.5). Only the second, third and fourth regimes are observed; the apparent absence of the first regime is due to its short duration. Furthermore, only the first three regimes will be addressed in detail; the fourth regime corresponding to isotropic spreading over the silicon oxide (see also Figs. $4.4(\mathrm{e}-\mathrm{i})$ ) is not considered here. 

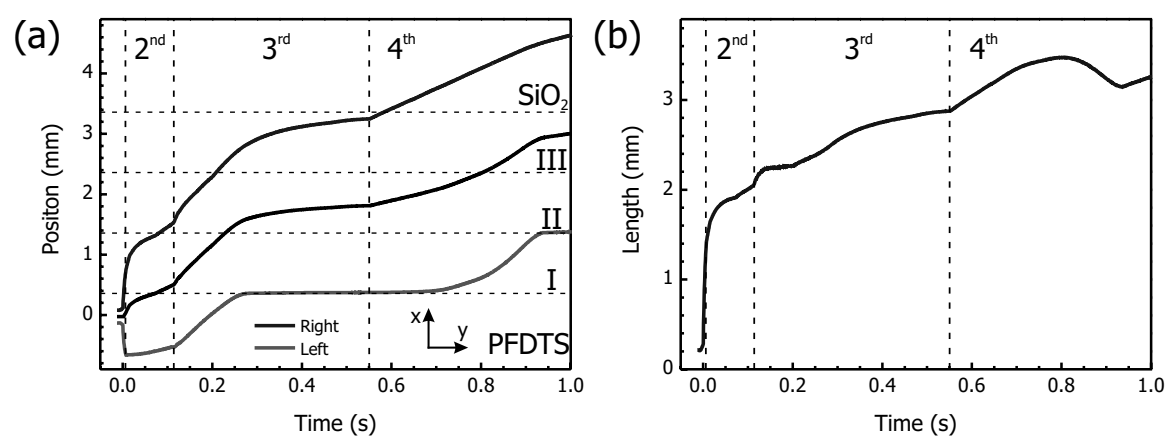

Figure 4.5: Evolution of the left (receding, red solid line) and right (advancing, blue solid line) edges as a function of time; the centre position (black line) is the average of both edges and in case of first, second and third regimes corresponds to the center of mass. The middle of the syringe is taken as the zero position on the surface; $x_{0}$ is uniquely defined for each experiment. A consequence of side view is reckoning that spreading starts from $200 \mu \mathrm{m}$ length $[44,63]$. Horizontal dashed lines indicate borders between the chemically defined patterns, as indicated on the right. Patterns I, II and III correspond to $\alpha=0.9, \alpha=0.5$ and $\alpha=0.3$, respectively. The vertical lines indicate transition between the motion regimes (second, third and fourth) as discussed in the text. (b) Time evolution of the droplet length in the $x$ direction for the same pattern.

As described in the experimental section, chemically defined patterns consist of PFDTS monolayers (transparent, approximately $0.7 \mathrm{~nm}$ thickness), which can not be observed by the naked eye. Experimentally, this results in scatter of the deposition position $x_{0}$ for different experiments on a scale smaller than $1 \mathrm{~mm}$. To enable comparison of the results obtained in different experiments, the time is set to 0s when the right side of the droplet crosses the border between the PFDTS rectangle and pattern I. Typically, this event occurs a few milliseconds after the droplet comes into contact with the surface.

It turns out that the initial position $x_{0}$ is highly relevant for the subsequent spreading kinetics. The distance $d$ is defined as the difference between $x_{0}$ and the border of pattern I (see also Fig. 4.2). Experimental values of $d$ vary from $0.2 \mathrm{~mm}$ up to approximately $0.7 \mathrm{~mm}$.

\subsubsection{First regime}

The first regime corresponds to the fast initial spreading of the droplet on a timescale $\left(\Delta \mathrm{t}_{1}\right)$ from $6 \mathrm{~ms}$ up to $10 \mathrm{~ms}$. This stage of spreading is gener- 

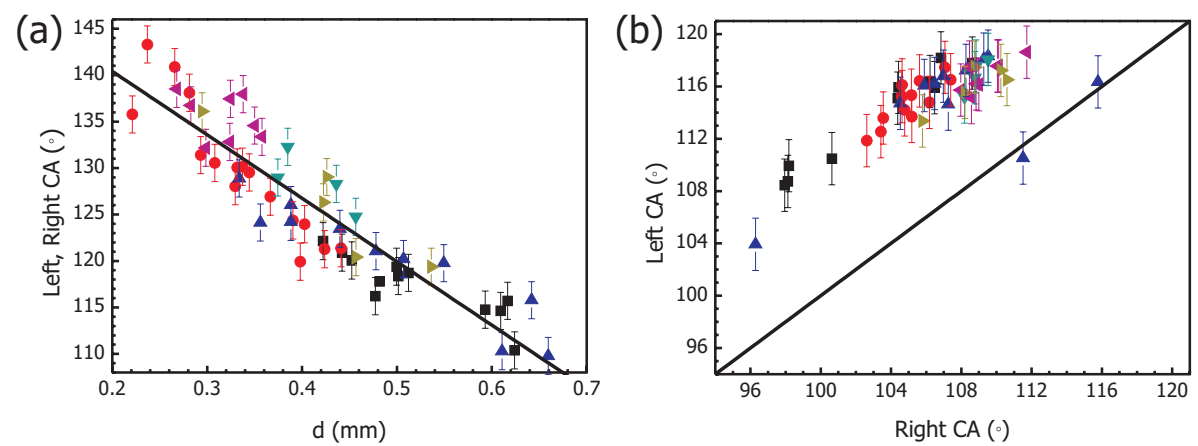

Figure 4.6: The results presented in this figure are extracted from all studied patterns with different individual stripe-pattern lengths and various combinations of $\alpha$-values. Each colour refers to a set of patterns having the same pattern I,II and III lengths and $\alpha$ combination, but different PFDTS and $\mathrm{SiO}_{2}$ stripe widths. Scaling of data suggests that the absolute width of stripes is not a relevant parameter for the description of motion.(a) Dynamic CAs at the moment the right side of the droplets reaches the border of pattern I plotted as a function of $d$ (solid line is a guide to the eye). The CA values on both sides of droplets are equal due to the symmetric circular shape of the wetted area. (b) The dynamic CAs on the left side, plotted as a function of those on the right side of the droplet, at the end of the first regime. The solid line represents the situation for symmetric droplets, i.e. identical CAs on both sides of the droplet.

ally referred to as the inertial regime [44, 53, 63]. The inertial regime starts when the droplet comes into contact with the substrate surface and ends when the radius of the wetted area reaches $90 \%(0.7 \mathrm{~mm})$ of the radius of the droplet suspended to the needle prior to deposition $(0.78 \mathrm{~mm})$. The power law behaviour of spreading as well as the involved timescales are consistent with previously published work. More importantly, no net translational motion is observed in the first regime. The liquid centre of mass remains on the symmetry axis defined by the middle of the needle.

During the inertial regime, the right side of the droplet advances over two distinct regions: (i) the hydrophobic PFDTS rectangle and (ii) pattern I consisting of alternating hydrophilic $\mathrm{SiO}_{2}$ and hydrophobic PFDTS stripes. As mentioned above, $d$ is between $0.2 \mathrm{~mm}$ and $0.7 \mathrm{~mm}$, which implies that the extent of spreading on the respective regions varies between experiments. To study the effect of spreading over two distinct surface areas, dynamic CAs are shown in Fig. 4.6. The values measured when the right edge of the droplet is at the border of the PFDTS rectangle are shown in Fig. 4.6 (a) as a function of the position where the droplet is deposited. Note that 
up to this moment the droplet is symmetric and CAs on both sides of the droplet are the same. When reaching the border of the first pattern, the values scatter around a straight line as shown in Fig. 4.6 (a). The decrease of the CA values from $145^{\circ}$ to $110^{\circ}$ with increasing $d$ values is in agreement with the expectations. For larger distances $d$ the spreading over the pure PFDTS area is more extensive. Lower contact angles are attained before the wetted area reaches the chemically defined border of the first pattern.

In Fig. 4.6 (b) the relation between CAs on both sides of the droplet at the end of the first, i.e. inertial regime are plotted. At this time, a part of the droplet is on pattern I, while the remaining part is still on the PFDTS. For completely symmetric droplets, the contact angles would be identical, and would correspond to the solid line in Fig. 4.6 (b).

The dynamic CA values at the end of the inertial regime exhibit a difference of $9^{\circ}$ between the right and left sides of the droplet. More specifically, the CAs measured on pattern I (right side of the droplet) exhibit lower values as compared to the ones on the PFDTS (left side). The systematically lower dynamic CA values for the right side suggest that the footprint of the droplet already becomes asymmetric in the inertial stage. The available data is insufficient to identify whether a distortion of the contact line on pattern I from a circular into an elongated shape has already taken place.

\subsubsection{Second regime}

Following the inertial regime, liquid-surface interactions take over and control the motion of the contact line in the subsequent regimes. We designate the regime between the end of the inertial spreading and the moment at which the neck connecting the droplet to the needle breaks as the second regime.

In this second regime, the left side of the droplet no longer advances in the negative $x$ direction and will start moving in the opposite, positive $x$ direction: from here on we address the left side as receding (Fig. 4.5 (a)). The right side continues to move over the pattern $\mathrm{I}$, and as such it is referred to the as the advancing side of the droplet. Overall, the centre of mass of droplet slowly shifts in the positive $x$ direction. Translational motion of the droplet under the influence of the underlying pattern starts in the second regime. From Fig. 4.5 (a) it is observed that the advancing motion over pattern I is faster as compared to the receding edge over the PFDTS, resulting in elongation of the droplet in the direction parallel to the stripes.

The duration $\Delta \mathrm{t}_{2}$ of the second regime typically varies from $50 \mathrm{~ms}$ to $200 \mathrm{~ms}$, increasing as a function of $d$. Nevertheless, an explicit trend can not be dis- 

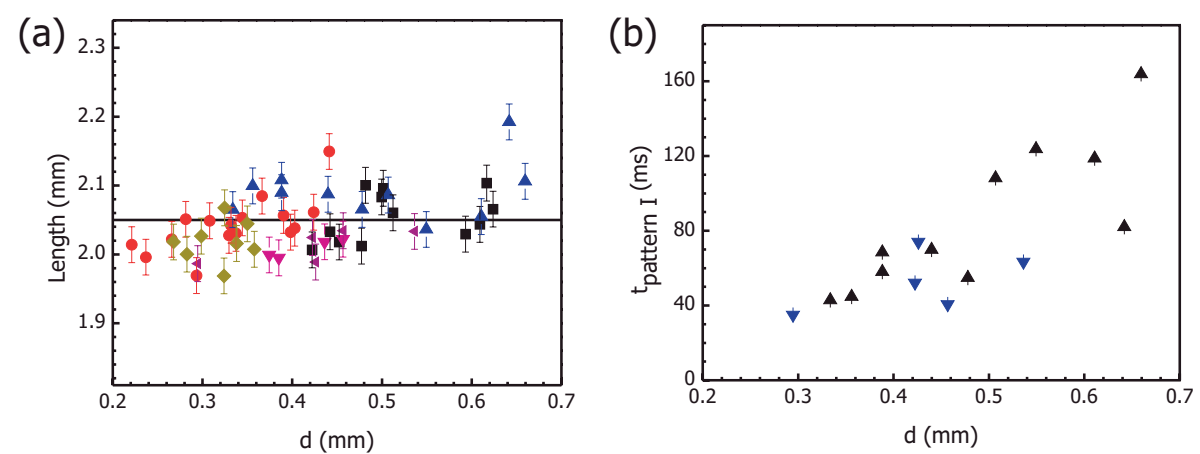

Figure 4.7: (a) Diameters of the droplet footprint in the $x$ direction, i.e. parallel to the stripes, at the moment of release from the needle as a function of $d$. The solid line is a guide to the eye. (b) Duration of time between the moments the advancing contact line enters and leaves the pattern I as a function of $d$. Only two datasets are presented pertaining to identical variables of $\alpha=0.9$ and the length of pattern I: the latter amounts $1000 \mu \mathrm{m}$.

cerned, suggesting that the release of the droplet from the needle does not depend on the time elapsed since the moment of contact with the substrate. To determine what triggers the release, the diameter of the footprint in the $x$ direction (referred to as the length of the droplet) at the moment of release is plotted as a function of distance $d$ in Fig. 4.7 (a).

It appears that experimental length values scatter around a mean value of $2.05 \mathrm{~mm}$ for all patterns studied. Apparently, attaining a specific length value is an essential parameter and triggers the release. This is consistent with the aforementioned increase of $\Delta \mathrm{t}_{2}$ as a function of $d$. The length of $2.05 \mathrm{~mm}$ cannot be achieved on pure PFDTS (on which a static diameter of $1.55 \mathrm{~mm}$ is observed), which requires that in most cases a significant part of the droplet volume shifts to pattern I. When the droplet is deposited further away from the border of pattern I, a larger part of the volume finds itself on the unpatterned PFDTS and needs to be moved to achieve the required length, resulting in durations of the second regime going up to 200ms.

From the high speed camera movies it is observed that for all droplets we have studied, the release event occurs once the advancing side has crossed the border between patterns I and II. In Fig. 4.7 (b) we plot the time $\Delta t_{\text {patternI }}$ that it takes for the advancing side of the droplet to move over the entire width of pattern I, as a function of deposition distance $d$. The data reveal a correlation between $d$ and $\Delta \mathrm{t}_{\text {patternI }}$. The time spent on pattern I increases 


\subsection{DISCUSSION}

with $d$, which implies that droplets deposited closer to the border of pattern I reach pattern II faster. Consequently, the droplets deposited close to the patterned area reach the required length for release from the needle in a shorter time.

\subsubsection{Third regime}

The third regime starts when the liquid neck connecting the droplet to the needle is broken and ends once the advancing side of the droplet reaches the border between pattern III and the unpatterned $\mathrm{SiO}_{2}$. During the third regime, the receding edge completely dewets the unpatterned PFDTS area, and becomes pinned at the border of pattern I. As a result, the droplet is stretched over all three striped patterns, exhibiting a shape resembling a cylinder with two hemispherical caps (Fig. $4.4(\mathrm{c}, \mathrm{d})$ ). The duration of the third regime varies between $300 \mathrm{~ms}$ and $700 \mathrm{~ms}\left(\Delta \mathrm{t}_{3}\right)$.

Immediately after the release event, an increase in speed of both the advancing and receding contact lines for a relatively short timescales is observed in Fig. 4.5 (a). The enhanced motion is more pronounced for the advancing side. It is difficult to conclude whether the release event induces spreading perpendicular to the stripes. In the top-view movies, no motion perpendicular to the stripes can be seen: the diameter in the $y$ direction remains approximately $1.7 \mathrm{~mm}$.

To check if the influence of the deposition position $x_{0}$ is still detectable, average speeds for the advancing contact line over the whole length of pattern III are calculated from the time differences between the moment at which the advancing contact line reaches the border of pattern III and the moment it starts spreading over the $\mathrm{SiO}_{2}$. Dividing by the length of pattern III yields speeds, which are approximately equal to $4 \mathrm{~mm} / \mathrm{s}$ for all studied patterns with different lengths of the various patterns. This confirms that in this stage of motion, there is no longer any influence of the deposition position.

\subsection{Discussion}

\subsubsection{Capillary spreading over striped patterns: second and third regimes}

The reason to separate the translational motion over the patterned surface into two regimes is motivated by the fact that (i) during the second regime the droplet moves while still attached to the needle, experiencing an addi- 
tional force which opposes the motion, and (ii) to investigate the influence of the release event on the non-restricted motion in the third regime.

In Fig. 4.3 the side-view snapshots taken from high speed camera movies depict the droplet motion during the first three regimes. More specifically, the images in Fig. $4.3(\mathrm{~b}-\mathrm{d})$ can be used to illustrate the influence of the connection with the needle on the translational motion during the second regime. The droplet is in the capillary regime, i.e. liquid-surface interactions drive the motion of the droplet (eq. 4.2); viscous drag (eq. 4.3) and hysteresis (eq. 4.4) forces restrict the motion. However, when the centre of mass of the droplet moves away from the symmetry axis of the needle, additional liquid-air interface area is created near the neck connecting the droplet to the needle (Fig. 4.3 (c)). Creation and subsequent increase of the additional liquid-air surface while the droplet moves away from the needle costs energy. This in turns gives rise to an overall slowing down of the droplet motion.

The important cost in energy for creation of this additional surface manifests itself in the enhancement in velocities of both advancing and receding contact lines after the connection with the needle is ruptured (Fig. 4.3 (d) and Fig. 4.5). The velocity enhancement can be qualitatively explained by considering the neck, which connects the droplet volume to the liquid reservoir within the needle, as a constrained spring. Once the spring is released, i.e. the neck breaks, the potential energy is liberated [53]. Subsequently, the potential energy is converted into kinetic energy, leading to a rapid increase of the elongation up to $25 \%$ of the value observed over the entire third regime in approximately $6 \%$ of time. More specifically, in $25 \mathrm{~ms}$, the droplet gains an additional $0.25 \mathrm{~mm}$ in length, see Fig. 4.5 . Once the velocity peak has passed, the droplet motion is controlled by the balance of three forces, resulting in overall decline of measured velocities to a few $\mathrm{mm} / \mathrm{s}$, in agreement with other publications $[76,77,81]$.

\subsubsection{Smart design}

The use of anisotropic striped patterns enables the creation of droplets with static shapes markedly different from the spherical and defines a preferential direction for spreading. Due to confinement of the contact line motion in the direction perpendicular to the stripes, the advancing motion parallel to the stripes is facilitated. Consequently, higher velocities of the contact line as compared to isotropic droplet spreading are typically observed.

Furthemore, in the direction parallel to the stripes the static CAs are reasonably well predicted by the Cassie-Baxter equation, rewritten in terms of 


\subsection{DISCUSSION}

the dimensionless parameter $\alpha$ (eq. 4.5):

$$
\theta_{\|}=\arccos \left[\frac{\alpha \cos \left(\theta_{\mathrm{PFDTS}}\right)+\cos \left(\theta_{\mathrm{SiO}_{2}}\right)}{1+\alpha}\right]
$$

where $\theta_{\text {PFDTS }}$ and $\theta_{\mathrm{SiO}_{2}}$ represent the CAs on homogeneous PFDTS SAMs $\left(105^{\circ}\right)$ and on $\mathrm{SiO}_{2}\left(23^{\circ}\right)$, respectively [85]. From this equation, it follows that for the pattern design considered in the previous section with $\alpha$-values of $0.9,0.5$ and 0.3 , corresponding static CAs are $68.8^{\circ}, 61.4^{\circ}$ and $49.5^{\circ}$, respectively.

The experimental dynamic CAs have been presented in Fig. 4.3. During the capillary stage, i.e. the second, third and forth regimes, the advancing CAs exhibit smaller values as compared to the receding ones. Also, the difference between CAs on both sides of the droplet is less than $10^{\circ}$, both being in agreement with the conditions given by eqs. $4.2,4.3$ and 4.4. Furthermore, comparing the experimental dynamic advancing CAs with the calculated static values (eq. 4.6), we find that the CAs at the end of each pattern are $>10^{\circ}$ higher.

Based on earlier studies reported in literature, in the case of chemically heterogeneous surface comprised of two different chemical entities, the advancing CA is defined by the more hydrophobic species, while the receding CA is governed by the more hydrophilic one [28]. For the unpatterned PFDTS SAMs the value of the advancing angle is approximately $5^{\circ}$ above the static value. We assume that this difference also holds for the stripepatterned surfaces. Based on this, taking into account that our experimental results for advancing CAs show values more than $10^{\circ}$ higher than the static CAs, the length of the striped patterns can be increased. Indeed, preliminary results on patterns with length of up to $1500 \mu \mathrm{m}$ (overall length of $4500 \mu \mathrm{m}$ ) have shown droplets moving over the entire design, and leaving it onto the unpatterned oxide surface.

As expected, changing the length of the patterned areas has immediate consequences on the droplet velocity during its motion. To illustrate this influence, in Fig. 4.8 we plot the time it takes the advancing edge of the droplet to move over the three striped patters; patterns with same $\alpha$ combination but different lengths are considered. First of all, the difference in timescales is surprising; the ratio of length scales does not correspond oneto-one to the time ratios. In the case of pattern lengths of $700 \mu \mathrm{m}$, i.e. the total length of the striped pattern amounts to $2100 \mu \mathrm{m}$, the overall time it takes the advancing droplet edge to wet all three patterns varies from $0.12 \mathrm{~s}$ to $0.3 \mathrm{~s}$ (Fig. $4.8(\mathrm{a})$ ), yielding average velocities typically above $10 \mathrm{~mm} / \mathrm{s}$. For patterns of length $1000 \mu \mathrm{m}$ (total striped pattern length $3000 \mu \mathrm{m}$ ) varia- 

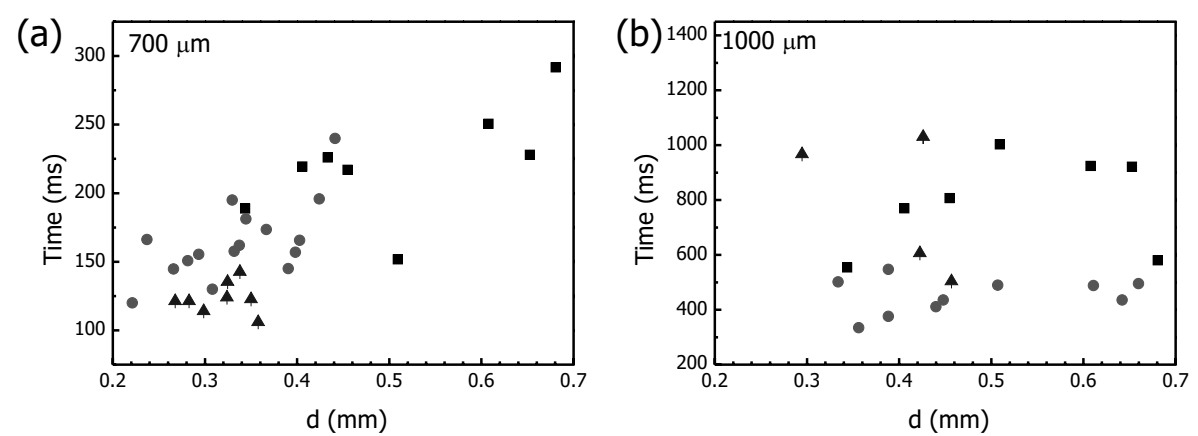

Figure 4.8: The time span for the advancing edge of the droplet to wet all three striped patterns presented as function of $d$. The results pertain to the patterns with the same values for $\alpha$, but different pattern lengths. The duration is obtained from the time difference between the moment at which the advancing edge reaches pattern $\mathrm{I}$ and the moment it reaches the border of the unpatterned $\mathrm{SiO}_{2}$. Results are shown for the individual pattern lengths of (a) $700 \mu \mathrm{m}$ (overall length of $2100 \mu \mathrm{m}$ ) and (b) $1000 \mu \mathrm{m}$ (overall striped pattern length of $3000 \mu \mathrm{m}$ ).

tions from $0.3 \mathrm{~s}$ to $1 \mathrm{~s}$ (velocities below $9 \mathrm{~mm} / \mathrm{s}$ ) are observed, while there is considerably more scatter of the data (see Fig. 4.8 (b)). As is to be expected, the time increases as a function of $d$ for both pattern designs. To identify a more obvious trend and enable a quantitative discussion between pattern dimensions and droplet velocities, additional experiments covering a wider range of pattern lengths need to be performed.

Another factor that influences the overall velocity of the droplet motion over the patterns is $\Delta \alpha$, i.e. the difference in macroscopic wettability, between patterns I, II and III. In agreement with intuitive expectation, for larger differences in $\alpha$, the droplet will wet and dewet the patterned surface more rapidly. During the experimental work presented in this chapter, for pattern I only values of $\alpha<1$ have been used, exhibiting static CAs below $68.8^{\circ}$. This in turn limits the possible combinations for velocity enhancements, since successive $\alpha$-values must decrease. However, based on observations of experimental dynamic CAs on the first pattern, CA values $\theta_{\mathrm{St}}=75^{\circ}$ are sufficient to initiate motion on this pattern. This indicated that the $\alpha$-value of the first pattern can be as high as 1.3, therewith enabling use of a wider range of possible combinations. The lower limit for $\alpha$ is imposed by the fact that the last pattern (pattern III) must have $\alpha>0.2$ for droplet to be able to dewet it. On experimental patterns III having $\alpha<0.2$ most of the time the receding motion of the droplet is inhibited; typically average velocities below $1 \mathrm{~mm} / \mathrm{s}$ were observed. 


\subsection{CONCLUSIONS}

Another reason in favour of using higher $\alpha$ values is the consequent increase in receding contact angles over pattern I. For all experimental sets, the receding motion over pattern I starts only when the advancing droplet edge initiates spreading on the $\mathrm{SiO}_{2}$, implying that the droplet to first wet all three patterns. Employing higher $\alpha$ values may allow dewetting of pattern I at an earlier stage of motion resulting in shorter residence times or enable using markedly longer overall pattern lengths.

\subsection{Conclusions}

We present a detailed experimental study of glycerol/water mixture droplets over chemically defined linear stripe-patterned surfaces. Droplets are gently deposited after which their motion induced by a surface energy gradient is investigated. Motion of droplets over distances larger than $3 \mathrm{~mm}$ on a timescale of seconds is observed. To enable a clear description and allow adequate discussion, the motion process is separated into four regimes; the first three are discussed in detail. Using patterns consisting of alternating hydrophobic (PFDTS) and hydrophilic $\left(\mathrm{SiO}_{2}\right)$ stripes gives rise to confinement of the motion in the direction parallel to the stripes, therewith enabling transport of liquid in a controlled way. Moreover, a precisely defined pattern design on the surface allows a quantitative estimate of the macroscopic surface energy at any position during movement. This makes it possible to ultimately refine pattern designs for application purposes. Furthermore, the dependence of droplets velocities on length and relative hydrophobicity of the patterns is discussed. Finally, chemically defined striped patterns seem to be a promising choice from an application point of view, owing to the combination of anisotropic spreading with enhanced contact line motion in the predefined direction as well as easily tunable surface energies. 



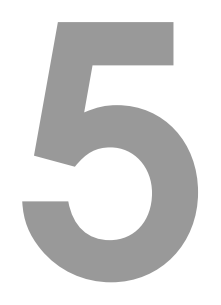

\section{Droplet motion over radially patterned surfaces}

The motion of droplets induced by chemically created anisotropic patterns is stud$i e d$. The patterns are created by hydrophobic fluorinated self-assembled monolayers (SAMs) on silicon substrates; the uncovered silicon comprises the hydrophilic parts of the pattern. The design consists of a central hydrophobic circle of unpatterned SAMs surrounded by rings of radial patterns which exhibit an increasing macroscopic wettability. The radial patterns consist of alternating hydrophobic and hydrophilic stripes; variation of their relative widths enables to design different wettability gradients. Once a droplet is deposited on the circle in the middle it will start to move over to the radially defined wettability gradient, away from the centre owing to the increasing relative surface area of hydrophilic matter for larger radii in the pattern. The focus of this Chapter is on a qualitative description of the characteristic motion on such types of anisotropic patterns. The effect of variation of the radial pattern lengths and stripe widths on the motion of the droplets is described. The results are discussed in terms of the design of the underlying pattern. 


\subsection{Introduction}

Inkjet printers encompass an extremely popular choice both for household purposes as well as for many different industrial applications. For domestic application the low price is a major advantage, while for industrial use the versatility of the technology is of prime importance, enabling printing of a wide range of materials on various substrates $[3,4,86]$. Most recently, the possibilities of printing were even extended to three-dimensional objects. The application areas for inkjet technology include displays [6, 87], the chemical industry [88, 89], electronic components [90-93], and a large emerging field comprises the life sciences [94, 95]. For all these applications a high speed and a high printing quality are required. This in turn has resulted in a lot of research activities in this area. Companies and research institutes developing the technology are faced with the challenge to further miniaturize and increase efficiency of the inkjet print heads.

The developments in silicon-based Micro Electro Mechanical Systems (MEMS) are presently also being applied to the inkjet printhead technology. For instance, in $200827 \%$ of all inkjet printheads were fabricated using MEMS technologies. Despite the substantial investments initially required, many printing companies incorporate MEMS into their products for massproduction, since this technology enables achieving sub-micrometer resolution with high reliability which is a must for large scale applications [12]. However, miniaturization of devices poses new challenges dictated by the fact that considerably more features have to be reproduced on markedly smaller surface areas [96].

The way the droplets are created in an inkjet printhead gives rise to small volumes of ink overflowing from the nozzles and ultimately wetting the nozzle plate $[3,16]$. Both metal and silicon nozzle plates are lyophilic for low surface tension inks, inhibiting complete removal of the residential ink by mechanical wiping. Accumulation of ink near the nozzle interferes with jetting of droplets and may lead to the interruption of jetting all together by blocking the nozzle [14, 15]. This in turns leads to unreliable jetting behaviour and is consequently detrimental for the print quality. Moreover, silicon nozzle plates as manufactured using MEMS technology, exhibit wettability characteristics, which are strongly affected by ambient conditions such as temperature and humidity. Additionally, the silicon surface is easily contaminated therewith creating pinning sites which interfere with ink removal procedures.

A smart way to avoid undesired ink accumulation near the nozzle is to apply an anti-wetting coating on the nozzle plate $[3,97,98]$. The surface is 


\subsection{EXPERIMENTAL DETAILS}

hydrophobized using a chemically inert agent which allows easy removal of ink and reduces contamination. Presently, such an antiwetting coating is primarily applied as a single chemical species homogeneously deposited over the entire nozzle plate. However, it may be relevant to design patterns of more (typically two) chemical species with different surface energies, which will enable controlling ink movement on the nozzle plate, ultimately achieving a continuous flow of ink away from the nozzle.

In this Chapter, a study of liquid behaviour on radially patterned surfaces is presented. A surface free energy gradient will induce droplet motion away from the point of the deposition. First, a global description of the droplet motion is given; similarities with previously discussed linear patterns (Chapter 4) as well as new features characteristic for radial patterns are discussed. Furthermore,the receding motion is treated in more detail, including a discussion of the residual layer covering the hydrophilic $\mathrm{SiO}_{2}$ after the droplet passage. The formation of liquid bridges covering up to 20 adjacent stripes at regular intervals and their influence on receding motion is presented. Finally, the influence of the radial pattern length on the motion velocity is presented.

\subsection{Experimental details}

\subsubsection{Surface preparation}

The surface patterns of self-assembled monolayers (SAMs) of $1 \mathrm{H}, 1 \mathrm{H}, 2 \mathrm{H}, 2 \mathrm{H}-$ perfluorodecyltrichlorosilane (PFDTS, 97\%, ABCR, Germany) on silicon wafers are created using standard clean room facilities. First, a positive photoresist is spin-coated on freshly cleaned wafers with a natural oxide film, followed by soft-baking. Patterns are created via standard optical lithography, after which the exposed photoresist is washed-off. The remaining photoresist is hard-baked and provides surface protection during vapour deposition of PFDTS; the silane head-group binds covalently to the native silicon oxide, exposing the fluorinated tail to the liquid. The assembly creates a densely packed layer of molecules with a height in the order of one $\mathrm{nm}$, on which glycerol has a stationary contact angle (CA) $\theta_{\mathrm{St}}=106^{\circ}$. Vapour deposition is done in a degassed chamber that is successively exposed to PFDTS and water reservoirs to introduce the respective vapours, initiating the reaction on the wafer surface [34]. After formation of the SAM, the photoresist is washed off, leaving a chemically patterned surface.

A typical layout used for the experiments in this Chapter is presented in Fig. 5.1. The central circle is formed by a homogeneous unpatterned 


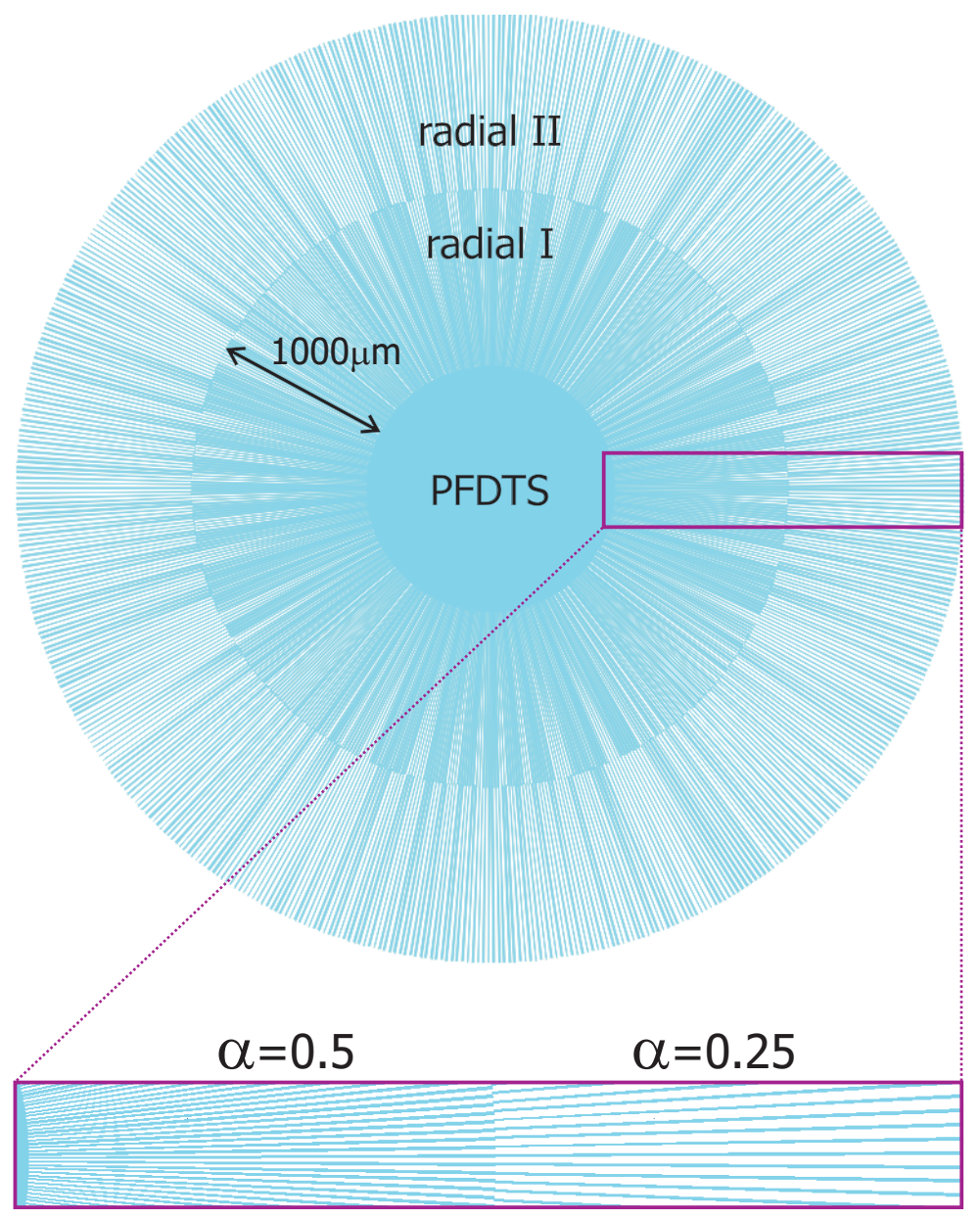

Figure 5.1: Lithographic mask used to create the radial pattern on silicon wafers. The droplet motion as presented in Fig. 5.2 and Fig. 5.5 were obtained using this particular pattern. The hydrophobic regions (PFDTS) and hydrophilic parts $\left(\mathrm{SiO}_{2}\right)$ correspond to the blue and white areas, respectively. The wettability gradient is created by the transition from the homogeneous hydrophobic PFDTS central circle to the unpatterned $\mathrm{SiO}_{2}$ via the circular radial patterns consisting of stripes of alternating wetting properties. A pattern typically consists of two or three radial patterns with lengths varying from $500 \mu \mathrm{m}$ to $1000 \mu \mathrm{m}$ and hydrophobic-tohydrophilic ratio $\alpha$ varying from 0.9 to 0.125 . As shown in the enlarged part, the width of stripes is not uniform. The stripes become wider with increasing distance from the centre. This is done in order to maintain a constant value for $\alpha$ (eq. 5.1) over the whole radial pattern length. Furthermore, the beginning and ending of the PFDTS stripes between radial patterns do not match. 


\subsection{EXPERIMENTAL DETAILS}

PFDTS SAM and has a diameter of $1.4 \mathrm{~mm}$ for all patterns studied. Droplets are always deposited on this circle. The circle diameter is chosen to be close to that of a $1 \mu 1$ glycerol droplet on an unpatterned PFDTS SAM $(1.39 \mathrm{~mm})$. However, as deposition exactly in the middle of the circle cannot be achieved (patterns are not visible by the naked eye prior to wetting), the droplet will encounter the more hydrophilic part of the pattern before the equilibrium shape is reached. Consequently, the droplet will be pulled away from the centre of the pattern and the translational motion is initiated. Surrounding the pure PFDTS circle are annular regions consisting of alternating hydrophobic (PFDTS) and hydrophilic $\left(\mathrm{SiO}_{2}\right)$ stripes with an increasing macroscopic surface energy. These create a preferential spreading direction for the droplets, similar to what has been described for linear patterns in the previous Chapter. The fraction of hydrophilic surface area increases for radial sections further away from the centre. As in the previous Chapters, we use a dimensionless parameter $\alpha$ to quantify the relative hydrophobicity of the pattern:

$$
\alpha=\frac{w_{\mathrm{PFDTS}}}{w_{\mathrm{SiO}_{2}}}
$$

where $w_{\text {PFDTS }}$ and $w_{\mathrm{SiO}_{2}}$ are the hydrophobic PFDTS and hydrophilic $\mathrm{SiO}_{2}$ stripe widths, respectively. Patterns with smaller values for $\alpha$ are more hydrophilic corresponding to higher surface energy.

\subsubsection{Droplet deposition}

Droplet deposition is done using an OCA 15+ goniometer (DataPhysics, Germany), employing a computer-controlled syringe. We use glycerol (ReagentPlus, Sigma, USA) for all experiments, employing its high viscosity: it takes tens of seconds for droplets to move over the patterned surface. Unless otherwise specified, for all droplets the volume is fixed to $1 \mu 1$. The variation in droplet diameter just after being produced from the syringe was measured to be less than 5\%. Deposition of the droplet is achieved by very slowly lowering the syringe with the suspended droplet toward the substrate until it contacts the patterned surface.

A colour usb camera is mounted above the deposition stage and is used to image the temporal evolution of the droplets over the patterned surface. Top-view movies are used to monitor and analyze droplet motion over the radial patterns. The top-view camera has a frame rate of 30fps, enabling a qualitative description of the slow movement of the glycerol droplets. After the outward movement of the droplet, a residual layer of glycerol 
remains on the patterned surface. The colour movies provide a means to monitor this residual film.

In contrast to the linear patterns described in the previous Chapter, the symmetry of the surface patterns with respect to the deposition point makes it virtually impossible to predict the direction of motion of droplets. Therefore, it is not possible to quantitatively monitor the droplet kinetics using a (high-speed) side-view camera.

\subsection{Results: Droplet motion overview}

\subsubsection{Advancing motion as compared to linear patterns}

In Fig. 5.2 a set of top view images is presented. Although the patterns are designed to study motion of $1 \mu 1$ droplets, to provide an overview of the motion we use a $5 \mu 1$ droplet. This allows a better time resolution using the top-view camera to study the characteristic features for these anisotropic patterns, as these larger droplets take longer, typically more than one minute, to move over the pattern. However, the fact that we are not able to use high-speed camera side-view images, makes it impossible to temporally define the first three stages of motion as described for linear patterns, nor to make a quantitative comparative study. In the following, we will limit ourselves to a qualitative description of motion to indicate similarities as well as discrepancies between the two types of patterns.

Furthermore, as the receding angle of glycerol on $\mathrm{SiO}_{2}$ is very small, a thin layer of glycerol remains on the $\mathrm{SiO}_{2}$ stripes, which allows us to trace the way the droplet evolves over the patterned surface. Contrary to linear patterns, much attention will be given to remaining traces to extract information regarding the motion process.

The diameter of a $5 \mu 1$ droplet on the pristine PFDTS SAMs amounts to $2.38 \mathrm{~mm}$. The translational motion of the droplet under the influence of the underlying chemically defined pattern is initiated once the droplet completely wets the first striped region, referred to as radial I. The motion direction will be defined by the position where the droplet first comes into contact with the central circle. If the initial contact of a completely symmetric droplet will be exactly in the middle of the pattern centre, no motion would occur.

As is observed in Fig. 5.2 (b), the deposition point is in the first quadrant, i.e. slightly above and to the right of the geometric circle centre, which defines the translation direction to be toward the top-right corner of the image. In contrast to the case of linear patterns (see Chapter 4), no pro- 


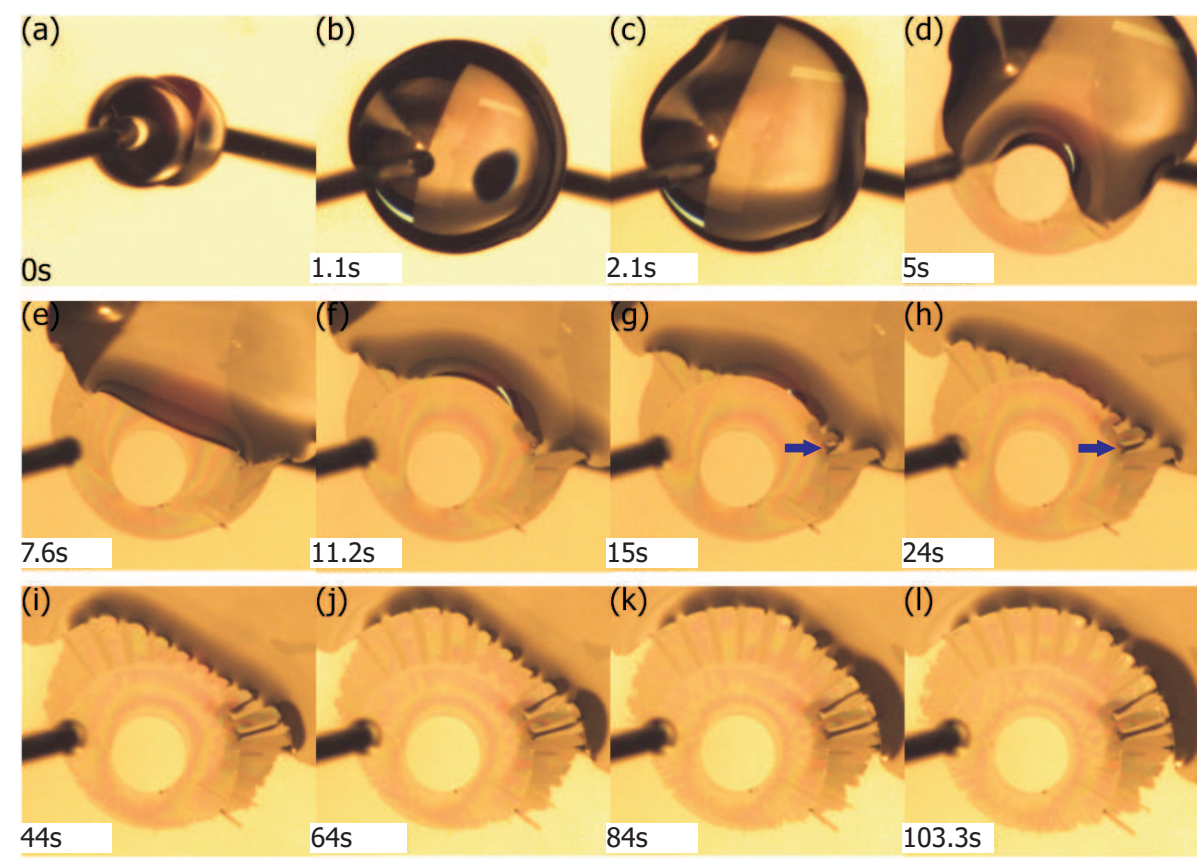

Figure 5.2: Motion of a $5 \mu 1$ glycerol droplet over a radially patterned surface. The diameter of the central PFDTS circle is $1.4 \mathrm{~mm}$; the two subsequently following radial patterns have $\alpha=0.5$ and $\alpha=0.25$ (referred to as radial I and radial II, respectively) and a length (i.e. the width of the annular region) of $1 \mathrm{~mm}$ each. The time values as indicated for every image are extracted from the top-view movie and provide an indication of the global timescale. (a) A suspended droplet $(r=1 \mathrm{~mm})$ is brought in contact with the central circle. (b) The droplet at the moment the translational motion is about to start, covering both radial I and radial II. The centre of mass is situated in the upper half of the PFDTS circle, defining the exit direction to be in the first quadrant. (c) The translational motion begins, followed by (d-f) dewetting of radial I. (g-l) Dewetting of radial II gives rise to liquid "bridges" (indicated by violet arrow) of identical size at regular intervals.

nounced confinement for the advancing contact line in the direction perpendicular to the motion in case of $5 \mu 1$ droplet is observed what is reflected in shapes close to spherical in Fig. $5.2(b, c)$. For smaller droplet volumes of $1 \mu \mathrm{l}$ (Fig. 5.5 (a-c), which will be discussed later), straight sections of the contact line in the direction perpendicular to the motion can be observed due to pinning at the PFDTS stripes, but their influence on the advancing motion should be minimal due to their position at the rear edge of droplet. Overall, the advancing motion of the droplets on our patterns with well- 


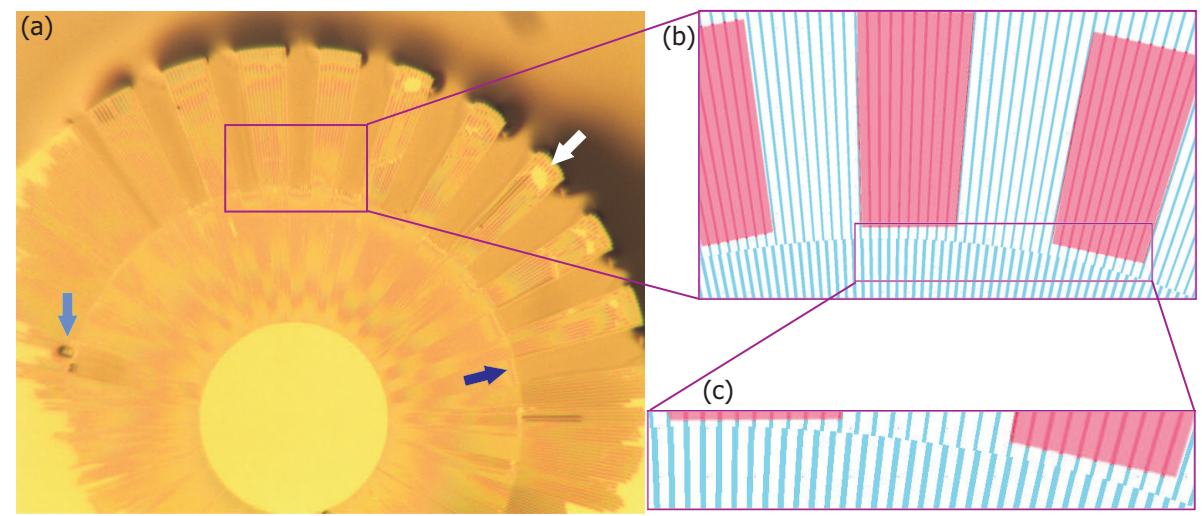

Figure 5.3: (a) Photo of a radial pattern with glycerol remaining on the $\mathrm{SiO}_{2}$ stripes after the droplet has passed. The image is obtained a few minutes after movie such as that represented in Fig. 5.2 (PFDTS circle diameter 1.4mm; radial I and II have $\alpha=0.5$ and $\alpha=0.25$, respectively; the width of the annular regions amounts to $1 \mathrm{~mm}$ each). The blue arrow indicates one of the liquid bridges on radial II. The white arrow shows the discontinuity in the wetted $\mathrm{SiO}_{2}$ stripes. The light-blue arrow indicates the square droplet formed by receding of the unstable bridge covering two $\mathrm{SiO}_{2}$ and one PFDTS stripes. (b) A corresponding part of the lithographic mask is shown. The blue stripes represent PFDTS, while the white colour refers to $\mathrm{SiO}_{2}$ stripes. The spatial locations where liquid bridges are observed, are indicated by the red shaded areas. For this particular pattern the bridges on average cover 10 periods of the pattern and are spaced by 10 periods. (c) The border between radial I and radial II shows the overlap between stripes in the two annular regions.

defined structure exhibits marked similarities with the behaviour of droplets on structureless energy gradient surfaces created by vapour deposition of molecules via diffusion-controlled process [99, 100].

\subsubsection{Droplets dewetting the radial pattern}

Similar to the linear patterns (Chapter 4), the motion of the receding edge is only observed when the advancing side starts to move over the unpatterned $\mathrm{SiO}_{2}$ (Fig. 5.2 (c)). The receding motion appears to be strongly influenced by the structure of the underlying pattern. Initially, the receding of the contact line is relatively fast over the PFDTS circle, followed by a considerable deceleration over the first annular region radial I. We assume this pronounced decrease in speed of the receding edge is due to pinning on the $\mathrm{SiO}_{2}$ stripes (Fig. 5.2 (d)). As a result, an unfavourable convex curva- 


\subsection{RESULTS: DROPLET MOTION OVERVIEW}

ture on the edge of the pure PFDTS circle and two strongly curved corners representing a high unbalanced Laplace pressure are formed on the receding side of the droplet. Due to the presence of these features, the receding velocity of the contact line is not homogeneous. Initially, the motion is only observed at the sharply curved corner regions, while the centre of the receding contact line remains pinned at the edge of the circular PFDTS region. However, this inhomogeneity in receding velocities along the contact line enables the droplet to adopt a more favourable shape with convex receding contact line. Similar inhomogeneity of receding velocities along the contact line is observed in case of $1 \mu 1$ droplets, though due to the smaller volume and faster droplet motion they are more difficult to identify.

The convex shape of the contact line will reappear when the contact line is pinned on the chemically defined border between the two annular regions, referred to as radial I and radial II (Fig. 5.2 (f)). Meanwhile, spreading over the $\mathrm{SiO}_{2}$ continues and the droplet volume is steadily being pulled off the patterned surface, enforcing the receding motion eventually of the outer patterned area designated as radial II.

Compared to the previously discussed linear patterns (Chapter 4), the droplets receding from the radial II pattern exhibits new features, which are emphasized by using a large volume droplet. A first observation is that the receding over the radial II pattern occurs on longer timescales as compared to the equivalent length and $\Delta \alpha$ of linear patterns. A second observation is the formation of the regularly spaced, symmetric liquid "bridges", covering up to ten periods (hydrophobic PFDTS plus hydrophilic $\mathrm{SiO}_{2}$ stripes) observed in Fig. 5.2 (g-1). Liquid bridges covering two or rarely three periods are occasionally observed on linear patterns; the occurrence and the position appear to be random and not reproducible between experiments. On the radial patterns, however, both width and position are reproducible. Furthermore, the presence of these bridges influences the receding contact line velocity.

A consequence of bridge formation or a discontinuous depinning of the receding contact line from the chemical border between radial I and radial II is that the definition of the overall receding velocity is complicated. In each separate receding part of the contact line, the liquid slides along the bordering bridge sides independently from neighbouring receding site. For example the liquid totally leaves the pattern in regions close to the droplet edges while in the centre it is still fully covering the radial II (Fig. 5.2 $(\mathrm{g}, \mathrm{h}))$. As a result, the global shape of the receding contact line can be close to straight as observed for the case of a $5 \mu 1$ droplet (Fig. 5.2 (h-j)), while for $1 \mu 1$ droplets, the curvature is more erratic as depinning is not synchronized 
(Fig. $5.5(\mathrm{k}-\mathrm{m})$ ). On linear patterns in most cases hemispherical shapes are observed.

Ultimately, the droplet volume completely dewets the radial pattern: a small amount of liquid which is left behind on radial II is located on the $\mathrm{SiO}_{2}$ stripes and in the liquid bridges, both being of microscopic thickness. A thin continuous layer of glycerol remaining on individual $\mathrm{SiO}_{2}$ stripes is stable and does not decay with time. The discontinuities in covering do not induce liquid rearrangements either, as can be seen on the enlarged image of the liquid trace as presented in Fig. 5.3. In a few interbridge spaces bright spots (one being indicated by white arrow) pertain to liquid-free surface areas.

Moreover, occasionally small bridges similar to the ones emerging on linear patterns covering two hydrophilic and one hydrophobic stripe between them can be observed. Contrary to linear patterns, these bridges appear to be unstable on radial patterns. Within seconds after formation the contact line situated at the wider region of stripes depins and the liquid recedes towards the centre, forming rectangular shaped droplets (Fig. 5.3, droplet residing near the border between radial I and radial II, indicated by the light-blue arrow). The reasons for their instability compared to larger bridges as well as the receding direction are not clear yet.

\subsection{Discussion}

\subsubsection{Liquid bridges occurrence on radially patterned surfaces}

As mentioned in the previous section, the combination of a low receding $\mathrm{CA}$ on the $\mathrm{SiO}_{2}$ stripes and the low vapour pressure of glycerol leads to a thin stable residual layer of liquid on the hydrophilic stripes after the droplet has passed. However, regularly spaced similarly sized liquid 'bridges' covering a few periods of the underlying pattern appear to be a common feature for all radial patterns. A better understanding why these bridges occur and why they are stable is of interest in particular for future pattern designs.

The image presented in Fig. 5.3 is obtained a few minutes after the droplet has moved over the pattern, allowing a study of the situation when the influence of the droplet is gone. The uneven borders both for the first and second radial section are seen (comparison of Fig. 5.2 (e) with Fig. 5.2 (l) shows filling of $\mathrm{SiO}_{2}$ stripes in radial II). Widening of the footprint of the droplet in an uneven way is observed due to various amount of the excess glycerol remaining on each $\mathrm{SiO}_{2}$ stripe. The system attempts to achieve 


\subsection{DISCUSSION}

the most favourable configuration by letting the excess liquid to wet the available hydrophilic surface. However, bridges persist without showing any sign of disintegration and dewetting of the unfavourable hydrophobic PFDTS stripes. Moreover, these bridges have varying widths and spacings as a function of the underlying pattern, as we conclude from the comparison of data obtained on various radial patterns.

An interesting observation is that none of the patterns show bridge formation on the first annular region, referred to as radial I. On the other hand, all patterns lead to the formation of regularly spaced bridges on the second annular region radial II. The bridges can be easily identified by the same monotonous colours as the bulk glycerol at distances exceeding the pattern radius. Individual $\mathrm{SiO}_{2}$ stripes covered with liquid exhibit red and yellow interference fringes, most likely due to thinner liquid films as compared to the height of the bridges. Moreover, a colour contrast is also observed on radial I when the covered $\mathrm{SiO}_{2}$ stripes of radial I are in contact with liquid bridges on radial II. Again assuming that the thickness of the liquid layer determines the optical response would indicate that different volumes of glycerol reside on hydrophilic stripes. As a matter of fact, the bridges can act as liquid reservoirs since they are connected to the macroscopic droplet outside the patterned area and can supply liquid to the stripes in the radial I.

In order to rationalize the occurrence of bridges on the second annular region designated as radial II, in Fig. 5.3 (b) and Fig. 5.3 (c) we depict the corresponding sections of the radial patterns. The similar sizes and regular positions of liquid bridges, i.e. their periodicity on various pattern designs suggests that the reason for their presence is most likely related to a repeatedly occurring feature in the patterns. By overlaying the top-view images with the lithographically defined pattern, it becomes clear that the bridges are observed in places where the hydrophobic and hydrophilic stripes of radial I and radial II are connected. However, for different $\alpha$ values in the two regions the periodicity of the stripes is different, and as such the relative positions of the stripes shift with respect to one another. In the region between the bridges the hydrophilic stripes of radial I end at the beginning of the hydrophobic stripes of radial II.

The fact that bridge formation is induced where the hydrophilic $\mathrm{SiO}_{2}$ stripes in the two regions are connected may well account for their absence on radial I (Fig. 5.4) as two different modes of receding motion may be considered. Prior to receding over radial I, the contact line is pinned at the border of the PFDTS circle, where there is no hydrophilic surface area (Fig. $5.4(\mathrm{a})$ ). As soon as the receding CA value for a given $\alpha$ is achi- 

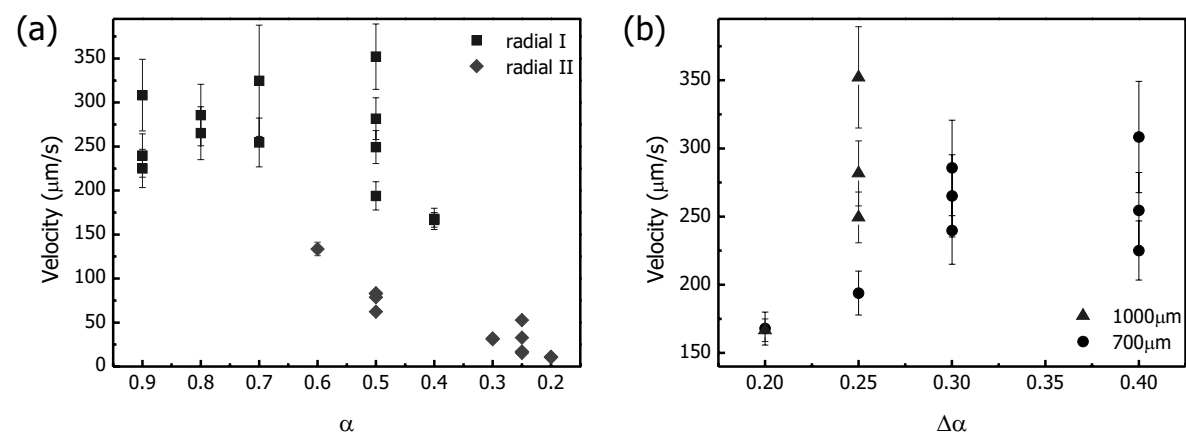

Figure 5.4: Schematic representations of contact line pinning at the chemical border (a) between the central PFDTS circle and radial I, and (b) between radial I and radial II. In (b) a part of the pattern is shown where bridge formation is observed and neighbouring regions without bridge: brige is schematically indicated by violet square. Blue regions correspond to hydrophobic PFDTS regions, while white represents the hydrophilic $\mathrm{SiO}_{2}$ surface. The direction of contact line motion is indicated by the arrows.

eved, the receding motion will be initiated simultaneously in places where PFDTS stripes are connected to the circle, entraining the liquid on the $\mathrm{SiO}_{2}$ stripes and consequently prohibiting bridge formation.

However, a different situation takes place once the contact line becomes pinned at the beginning of radial II as schematically shown in Fig. 5.4 (b). In this case not only the fraction of hydrophilic surface area on radial II is higher, which lowers the macroscopic receding CA, the shape of the contact line is also very different and not homogeneous along the border. In the regions where the bridges will be formed or where connected $\mathrm{SiO}_{2}$ stripes are present, the contact line oscillates regularly, partly remaining on the radial I. Given the continuity of the hydrophilic region combined with relatively smaller proportion of hydrophobic matter, on this portions of the pattern the receding motion will not be initiated until the CA reaches values close to zero (Fig: 5.4 (b)). However, in the regions where bridges are not formed, the hydrophobic stripes in radial II start in the centre of the hydrophilic stripes, perturbing the contact line structure, which in turn will promote the receding motion.

Further enlargement of the border between the two regions (Fig. 5.3 (c)) can indicate what is the critical mismatch between the respective hydrophobic stripes that will inhibit bridge formation and lead to separately covered $\mathrm{SiO}_{2}$. In this particular example, the bridges are stable as long as the PFDTS stripes of radial I are connected to ones of radial II. However, in some cases, 


\subsection{DISCUSSION}

the bridges persist once the PFDTS stripes are no longer connected, but are in the vicinity of one another. That arises due to limitations of the lithographic process: the mask shows sharply defined edges, while in the actual pattern the photoresist will smoothen out the features, creating a more gradual transition and allowing liquid to stay connected for the last $\mathrm{SiO}_{2}$ stripes.

Overall, we conclude that bridges will be stable in areas where the hydrophobic stripes of the subsequent, outer radial pattern are connected to the ones in the more inner radial region. When the hydrophobic sections of neighbouring striped regions are no longer connected, the corrugation of the contact line will be sufficient to initiate its receding motion.

\subsubsection{Kinetics of the receding motion}

As mentioned earlier, the large $5 \mu 1$ droplet is used mainly to show the way in which the droplet recedes over radially patterned surfaces as well as to highlight the repetitive nature of the bridge formation as a larger surface area is wetted. In the following, the discussion will concentrate on motion of $1 \mu 1$ droplets as in this case the influence of the underlying pattern is more pronounced.

In Fig. 5.5, the motion of a $1 \mu 1$ glycerol droplet over the radial pattern is shown. In this case, it takes approximately 16s for a droplet to leave the pattern. Moreover, due to the smaller volume the influence of the underlying structure resulting in straight sections of the contact line and the shape of the droplets is easier to be identified.

Taking into account the time it takes the droplet to dewet each respective radial pattern, another important reason for the absence of bridges on the first annular region radial I can be suggested. Other than the difference in the microscopic structure of the contact line, the difference in velocities of the receding motion over the subsequent radial areas is markedly different. In case of the $1 \mu 1$ droplets, the time span to dewet radial I is of the order of $3 \mathrm{~s}$, while it takes close to 10 s to dewet radial II.

Returning to the large droplet, the timescales for dewetting of radial I being close to 10 s, i.e. approximately 3 times larger than for the small droplets, can be explained by the fact that in the $1 \mu 1$ case the droplet recedes exclusively in the direction parallel to the stripes, while a large part of the $5 \mu 1$ droplet recedes across the stripes.

As mentioned, the time it takes to dewet radial II is almost eight times longer than that of radial I. This is most likely due to the discontinuous receding motion of the contact line and bridge formation (Figs. 5.2 (g-h) 
(a)

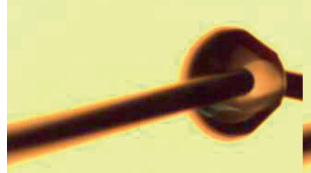

$0.2 \mathrm{~s}$

(e)

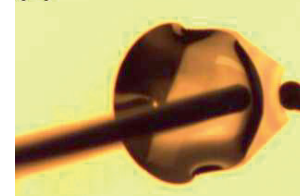

$1.9 \mathrm{~s}$

(i)

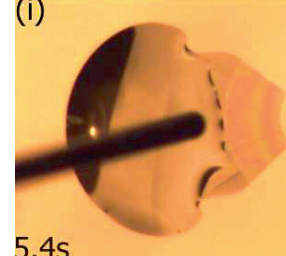

(b)

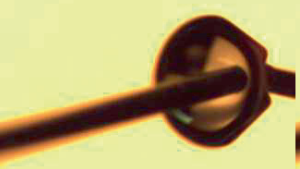

(c)

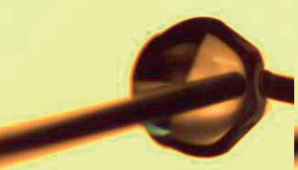

(d)

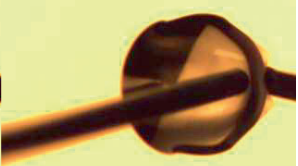

1s

(h) (f)

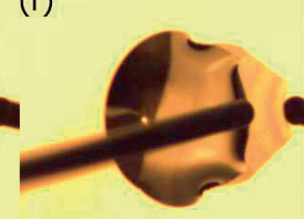

(g)

$2.6 \mathrm{~s}$

(j)

$3.4 \mathrm{~s}$
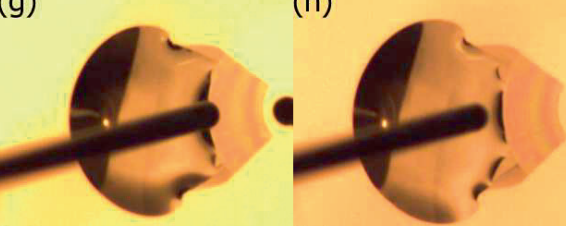

$4.4 \mathrm{~s}$

(I)

(k)

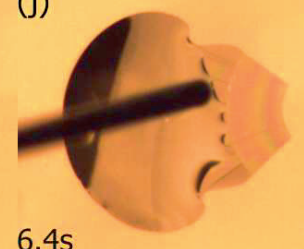

6.45

$8.4 \mathrm{~s}$
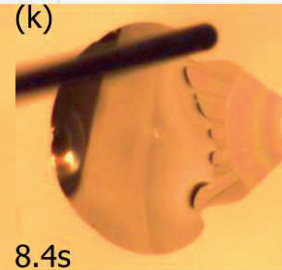

$15.4 \mathrm{~s}$

Figure 5.5: Motion of a $1 \mu 1$ glycerol droplet over a radially patterned surface. The pattern is identical as that used in Fig. 5.2 for the $5 \mu 1$ droplet. More specifically, the diameter of the central PFDTS circle amounts to $1.4 \mathrm{~mm}$; the two annular regions have $\alpha=0.5$ (radial I) and $\alpha=0.25$ (radial II), while their width (length in the radial direction) amounts to $1 \mathrm{~mm}$ each. The unsharp edge of the droplet in the first four images is caused by the relatively high contact line velocity in relation to the shutter speed.

and Figs. 5.5 (h-1)). As shown, the receding droplet edge depins inhomogeneously from the chemically defined border between radial I and radial II, creating necklace-like structures. Generally dewetting of radial II starts at edges of droplets and finally reaches the central region. Subsequently, liquid bridges as described in the previous section are remaining, while the contact line in the regions between the bridges recedes over the whole extent of radial II. To enable assessment of the timescale for the liquid to recede over radial II, we choose to define the duration of the receding motion to start once the last receding part becomes depinned from the border until the droplet completely dewet the regions between the bridges. 

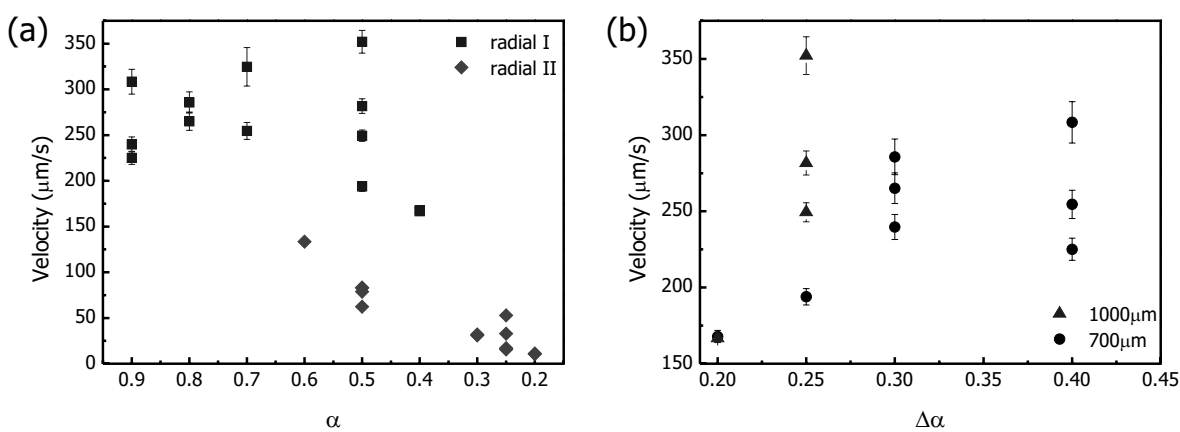

Figure 5.6: (a) Experimentally observed dewetting velocities for $1 \mu 1$ glycerol droplets over the radially patterned surfaces. The $\alpha$-values are presented in decreasing order to match the way they appear in the patterns. For both patterns the moment of depinning is taken once the contact line completely leaves the border. The moment of depinning is defined within three frames, corresponding to an error of $0.3 \mathrm{~s}$. The error bars for velocities on radial II are not discernable due to the large timescale. (b) Average velocity of the receding contact line over radial I as a function of the difference in $\alpha$-values between radial I and radial II. Here the effect of radial length of the pattern is considered.

\subsubsection{Surface energy dependence of the receding motion}

To enable a comparison of the difference in timescales of dewetting of various patterns with different surface energies represented by various $\alpha$ values for radial I and radial II, in Fig. 5.6 we plot the average velocities of the contact line as determined from the top-view movies. Presenting experimental data as a function of $\alpha$ has the advantage that patterns with variable lengths for radial I and radial II, as well as different combinations of $\Delta \alpha=\alpha_{\text {I }}-\alpha_{\text {II }}$ can be shown in a single plot.

The data in Fig. 5.6 (a) shows that the receding velocities for the radial I to exhibit similar values for different $\alpha$, but the scatter is large. A velocity increase for larger $\alpha$ values can be discerned for radial II, similarly to the linear patterns, but more experimental data is required for confirmation and identification of the specific trend. Moreover, for all patterns a marked difference in velocities over radial I and radial II is typically observed. However, in case of linear patterns the difference in receding velocities between subsequent patterns is observed to be smaller: typically velocities have comparable values or sometimes a difference of a factor 1.5. The reason for the less marked difference arises from the fact that the receding motion from the last linear pattern onto the unpatterned $\mathrm{SiO}_{2}$ occurs on a shorter timescales. The discrepancies between radial and linear patterns 
with similar values for $\alpha$ and length can be ascribed to the presence of the bridges, which substantially slow down the dewetting processes over the patterns.

To study if the influence of the pattern length as well as variation of the $\alpha$-value for radial II has influence on receding motion over radial I, in Fig. 5.6 (b) we plot the average velocity of the receding contact line as a function of $\Delta \alpha=\alpha_{\mathrm{I}}-\alpha_{\mathrm{II}}$. Two studied pattern lengths are indicated by different symbols. For both pattern lengths $(1000 \mu \mathrm{m}$ and $700 \mu \mathrm{m})$, the results show an increase of the velocity for larger differences $\Delta \alpha$ between the two radial regions. Such an increase is in agreement with expectations, as a larger $\Delta \alpha$ implies a larger difference in surface energies, ultimately leading to the faster movement of the droplet to the more favourable areas on the surface: similar trend is observed for linear patterns.

However, it is hard to draw conclusions concerning the influence of the pattern length on the velocities, as experimental data exhibits a lot of scatter. The present results on a limited number of patterns, indicate that the velocities are higher for patterns with $1000 \mu \mathrm{m}$ radial pattern length. An inverse trend is observed for linear patterns in Chapter 4. To account for these discrepancies quantitative studies are to be carried out on patterns with improved designs where the direction of the droplet motion is more controllable and high-speed camera based studies should be performed.

\subsection{Conclusions}

In summary, the qualitative description of glycerol droplet motion over radially patterned, chemically functionalized surfaces has been presented. The patterns considered in this Chapter are created by a hydrophobic PFDTS circle in the centre of the pattern design and surrounding it with annular striped regions consisting of radially oriented stripes of alternating wettabilities, i.e. hydrophilic and hydrophobic. The anisotropic surface patterning creates a preferential direction for droplet motion away from the hydrophobic center circle onto the hydrophilic unpatterned $\mathrm{SiO}_{2}$. The relative widths of the stripes (in the micrometer range) are varied to study droplet behaviour on patterns with variable macroscopic surface energies. The experimental results are compared with previous observations on linear patterns on which quantitative studies were carried out. Similarities as well as differences between the radial and linear patterns are described and discussed. Comparing the receding velocities, we find that overall the motion over radial patterns is slower as compared to that over the linear design, which is most likely due to the smaller extent of confinement im- 


\subsection{CONCLUSIONS}

posed on the shape of the droplets by the radial patterns. Furthermore, specific features observed on radial patterns, such as liquid bridges and residual liquid layers on individual hydrophilic stripes that remain after the droplet has receded over the pattern, are discussed to identify their origin and with the distant goal to avoid their formation in future pattern designs. 



\section{6}

\section{Metastable droplets on shallow-grooved hydrophobic surfaces}

The equilibrium shapes of water droplets on shallow-grooved hydrophobic surfaces are studied experimentally. The dependence of the two final states, notably metastable Cassie-Baxter and Wenzel, on the underlying geometric pattern is analysed and discussed. Surprisingly, in contrast to theoretical expectations, a significant portion of the droplets are in Cassie-Baxter state. The anisotropy of the patterns, defined by the relative groove and ridge widths, allows studying the influence of different mechanisms of spreading in orthogonal directions on the final shape of the droplets. The validity of the Cassie-Baxter and Wenzel models in case of anisotropic surfaces is investigated, comparing the experimental data with theoretical predictions in the two respective regimes. The influence of varying ridge widths for fixed groove widths on the final state adopted by the droplets, i.e. Cassie-Baxter or Wenzel, is discussed. 


\subsection{Introduction}

The behaviour of liquids on surfaces, which have been modified in a controlled way both structurally and chemically, is of great interest from a theoretical as well as a practical point of view. Of particular interest are studies on "Lotus leaf" or superhydrophobic surfaces, on which a liquid will have large contact angles (CAs), and roll off easily making them exhibit self-cleaning properties [23, 26, 101]. The range of applications for these superhydrophobic surfaces extends from car windows to microfluidics. This in turn explains the amount of research dedicated to designing robust Lotus surfaces as well as studying the behaviour of liquid droplets they support. Despite the vast amount of work being carried out in this field of research, numerous questions remain unanswered regarding the influence of particular surface structures on the equilibrium shape as well as, especially, dynamic behaviour of liquid drops.

Summarizing the experimental and theoretical research until now, on hydrophobic rough surfaces $\left(\theta_{\mathrm{St}}>90^{\circ}\right)$ the liquid droplet can end up in two distinct equilibrium states: (i) the Wenzel state, in which all asperities are filled with liquid or (ii) the 'fakir' or Cassie-Baxter state, in which air pockets are trapped in the structures beneath the droplet. Both states effectively increase the apparent $\mathrm{CA}$ as denoted by $\theta_{\mathrm{W}}$ or $\theta_{\mathrm{CB}}$, respectively, for the two aforementioned states. However, for the so-called Lotus effect, the 'fakir' state is required.

When a liquid fills all of the underlying structural features, there is a complete wetting of the liquid-solid interface. This leads to an increase of the total wetted area with respect to the flat surface. In this case the Wenzel equation [102] is used to estimate the apparent macroscopic CA $\theta_{\mathrm{W}}$ :

$$
\cos \theta_{\mathrm{W}}=r \cos \theta_{\mathrm{St}}
$$

where $r$ is the roughness factor, defined as the ratio of the actually wetted surface to the projected flat area under the droplet; $r$ is always greater than 1 . The angle $\theta_{\mathrm{St}}$ corresponds to the Youngs CA the liquid assumes on same smooth, chemically homogeneous surface.

In the Cassie-Baxter or 'fakir' state, the droplet rests on the tops of surface asperities, trapping pockets of air within the structure. The CassieBaxter equation [38] is used to estimate the apparent macroscopic $C A \theta_{\mathrm{CB}}$ for a droplet on a composite surface consisting of air and a hydrophobic solid:

$$
\cos \theta_{\mathrm{CB}}=f_{\mathrm{s}}\left(\cos \theta_{\mathrm{St}}+1\right)-1
$$




\subsection{INTRODUCTION}

Here $f_{\mathrm{s}}$ represents the fraction of the liquid interface that is in contact with the solid as compared to the projected surface area [103].

In a number of studies dealing with morphologically structured, hydrophobic surfaces, the Cassie-Baxter state appears to have a higher energy as compared to the Wenzel state. In other words, complete wetting of the surface structures corresponds to a situation of thermodynamic equilibrium. Nevertheless, the metastable Cassie-Baxter state is frequently observed on these surfaces due to the fact that the droplets are deposited from the top, effectively experiencing a local energy minimum [104-106]. The presence of an activation energy between metastable and thermodynamic equilibrium situations gives rise to droplets residing in the Cassie-Baxter state without spontaneous decay into the energetically more favourable Wenzel state. To gain insight in the transition mechanism, complete wetting of the structures can be induced by an increase of the Laplace pressure for example arising from evaporation [107, 108], by application of an electric voltage [109], or vibrations [110]. Though various approaches, supported by experimental data, have been suggested to model the transition [111-117], the exact mechanism remains elusive.

The aforementioned studies of the relative stability of the Cassie-Baxter and Wenzel states, as well as the transition from one state to the other, are typically performed on well-defined, isotropically structured surfaces. Highly appealing are surfaces with an anisotropic pattern that favours spreading of liquid in certain directions and hindering spreading in other directions. This generally leads to static droplet shapes deviating from spherical ones. Understanding of droplet dynamics on such surfaces is of considerable interest both from a theoretical as well as an application point of view [44]. Anisotropic surfaces are abundant in nature [118, 119]; their artificial equivalents with controlled structures can be used to study the occurrence of both states as well as their interdependence. Nevertheless, such studies prove to be rare owing to the difficulty to analyze the directional wetting characteristics. Consequently, the range of applicability of Cassie-Baxter and Wenzel equations in case of anisotropic surfaces remains an open question. The most often investigated anisotropic surfaces consist of parallel grooves, being attractive for their relative simplicity in modelling the results and the wide range of possible applications. The studies are typically carried out either for complete wetting, i.e. systems in the Wenzel state $[29,120,121]$ or for liquid droplets suspended on top of the grooves, i.e. the Cassie-Baxter state [122].

In this chapter we present an experimental investigation into the behaviour of liquid droplets on shallow groove-patterned hydrophobic sur- 
faces. Calculations to estimate the relative stability of the Cassie-Baxter and Wenzel states using actual geometric parameters reveal that for all surfaces the equilibrium situation corresponds to the Wenzel regime. Nevertheless, we observe systematically that droplet adopt either Cassie-Baxter or Wenzel states depending on the underlying pattern. Furthermore, the influence of the anisotropic pattern on the final shape and macroscopic CAs are studied for both regimes and compared to what is expected on the basis of the aforementioned models.

\subsection{Experimental details}

\subsubsection{Surface preparation}

We prepare our surfaces using standard clean room methods as schematically shown in Fig. 6.1. First, the oxide layer is removed from the Si wafers to assure an identical thickness of native oxide, therewith providing better homogeneity between different batches of wafers. Next, freshly cleaned wafers are spin-coated with positive photoresist. Subsequently, the photoresist is soft-baked and the pattern is transferred via standard optical lithography (Fig. 6.1 (a)). Once the exposed photoresist is washedoff, the remaining photoresist is baked. The exposed Si regions are etched by reactive ion etching (RIE) [123]. Anisotropic etching is used to create well-defined profiles of grooves of $2 \mu \mathrm{m}$ deep (Fig. 6.1 (b)). After removing the photoresist, the depth and the homogeneity of the etching at different places on wafers are assessed using a profilometer (Veeco Dektak 8). Finally, the wafers are thoroughly cleaned in nitric acid to prepare for hydrophobization of the surface with a self-assembled monolayer (SAM) of $1 \mathrm{H}, 1 \mathrm{H}, 2 \mathrm{H}, 2 \mathrm{H}$-perfluorodecyltrichlorosilane (PFDTS, 97\%, ABCR, Germany) (Fig. 6.1 (c)).

The assembly of molecules creates a densely packed thin layer with a height in the order of one nm, on which water has a static CA $\theta_{\mathrm{St}}=110^{\circ}$. On our experimental surfaces we measured static CA $\theta_{\mathrm{St}}=109^{\circ}$ (averaged over 8 independent measurements on flat parts of the wafer), confirming the good quality of our SAMs. Vapour deposition of the PFDTS molecules is done in a degassed chamber that is exposed in successive turns to PFDTS and water reservoirs to introduce the respective vapours, initiating the reaction on the wafer surface. Using a controlled environment ensures good quality and reproducibility of the SAMs [34]. 

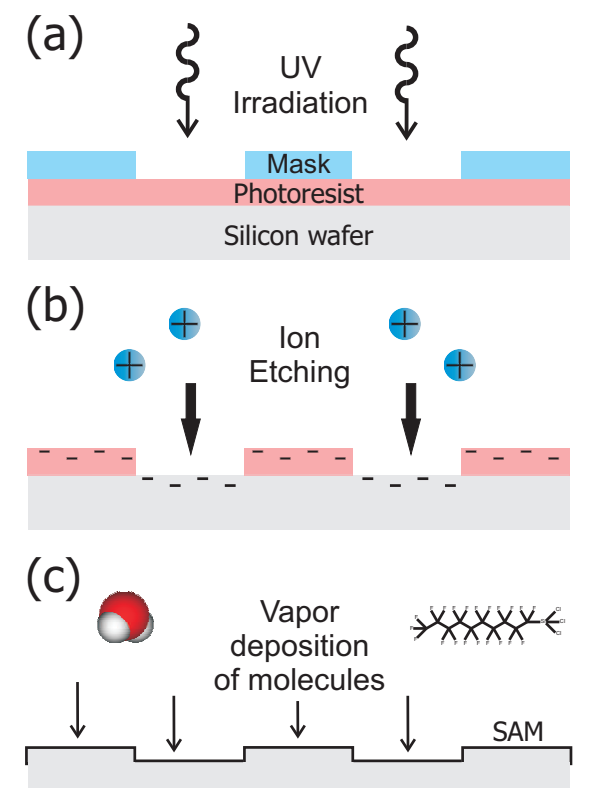

Figure 6.1: Schematic representation of the different surface preparation steps. (a) A photoresist layer on the $\mathrm{Si}$ wafer is exposed to light through a mask. Parts of the positive photoresist that are exposed to the light will be washed away, therewith creating a pattern on the surface. (b) Parts of the wafer that are not protected by photoresist will be removed by reactive ion etching (RIE) [123]. (c) After removal of the photoresist, a self-assembled monolayer of PFDTS is deposited. Wafers are placed in a glass chamber that is degassed below 50 mbar (vapour pressure of PFDTS). Subsequent steps are: (i) the chamber is connected to a reservoir containing liquid molecules, introducing PFDTS; (ii) the chamber containing PFDTS vapour is connected to a water reservoir, introducing water vapour, initiating silanization on the surface of the wafers [34].

\subsubsection{Droplet deposition}

Droplet deposition and characterization, including measurements of CAs, is done using an OCA 15+ apparatus (DataPhysics, Germany). The equipment enables determination of CAs with an accuracy of $0.5^{\circ}$. Droplets are created using a computer-controlled syringe. The liquid used is high purity water (from a Millipore Simplicity 185 system). For all droplets the volume is fixed at $1 \mu 1$.

Deposition of droplets for part of the patterns (the relatively more hydrophilic substrates) is achieved by gentle lowering of a syringe with a suspended droplet until it contacts the surface. The droplet spreads on 
the surface while still remaining attached to the needle. The detachment from the needle is induced by manual retraction of the syringe. Due to the relatively small volume of droplets in our experiments, the gravitational influence can be neglected.

For other patterns (relatively more hydrophobic), gentle lowering of the syringe will give rise to spreading of the droplet due to the pressure applied by the needle. However, when the needle is retracted the droplet remains attached to the needle and detaches from the surface. In these cases, the deposition was done by dropping droplets from a low height.

An additional camera is mounted above the substrate to assess the final shape of the droplets. Using the reflected light, it is possible to see through the droplet and view the liquid interface with the solid; the droplet effectively works as a magnifying glass. This property can be used to confirm the final state of the droplet as the light reflects differently from watersolid and water-air interfaces; typical examples are presented in Fig. 6.2 and Fig. 6.6. In the Cassie-Baxter state (Fig. 6.2 (a) and Fig. 6.6 (a)) the airliquid interfaces suspended above the grooves appear to be bright due to light scattering as compared to the dark liquid-solid interfaces of the contact regions. In the Wenzel state (Fig. 6.2 (b), and Fig. 6.6 (b)) the wetted area appears to have a more uniform colour as the grooves are filled with liquid; the grooves appear to have a slightly darker shade as less light reflects from the sides of the grooves.

\subsection{Results}

Depending on the ridge width, while keeping the groove width constant, the droplets end up in different static situations: either the Cassie-Baxter or Wenzel states are adopted. As a general rule, for patterns with ridge widths smaller or equal to the width of the grooves, the final state adopted by the droplets is the Cassie-Baxter regime. As the ridge width becomes larger than the groove width, droplets end up in the Wenzel state.

An example of a droplet in the Cassie-Baxter state is presented in (Fig. ?? (a)). The droplet has a spherical shape, reflecting little influence from the underlying anisotropic pattern. Indeed, the side-view images show that CAs $\left(\theta_{\|}\right.$and $\left.\theta_{\perp}\right)$ and diameters both perpendicular and parallel to the grooves $(N$, resp. $P)$ have similar values.

The second set of photos (Fig. 6.2 (b)) shows the droplet in the Wenzel state. From the top-view, the elongation in the direction of the grooves can be observed, similarly to what was previously described for chemically patterned surfaces $[44,85]$. The side view photos show the difference in 


\subsection{RESULTS}

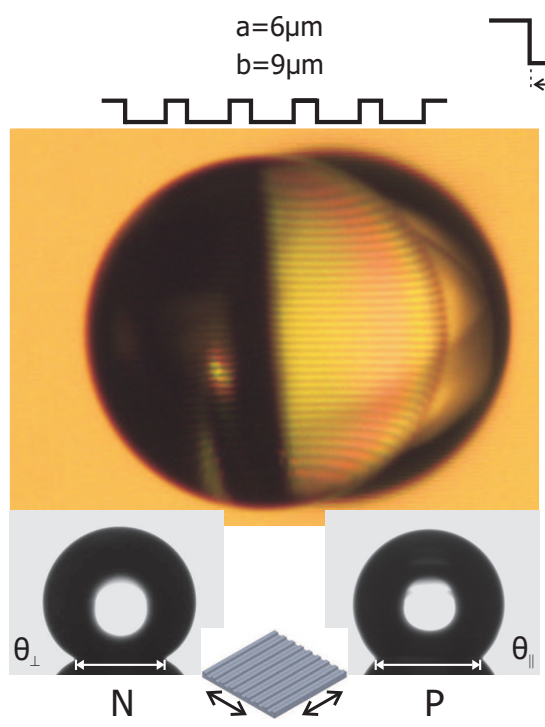

(a)

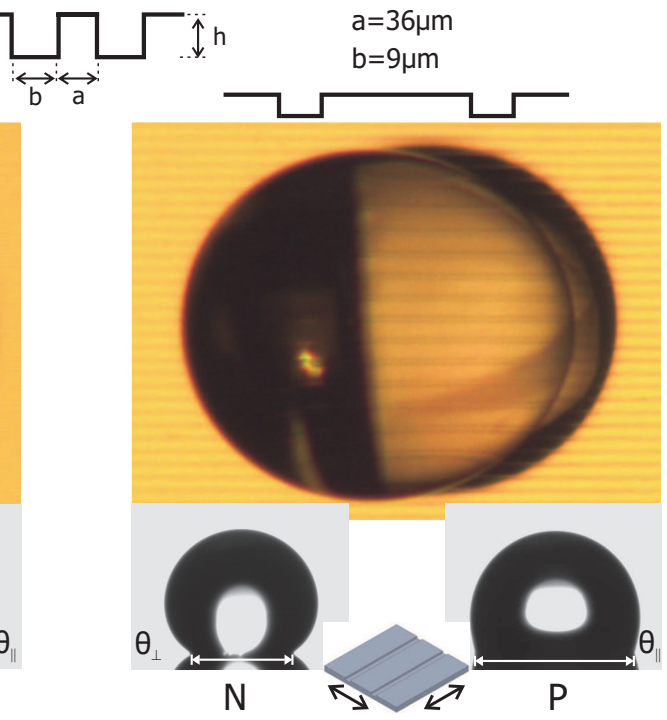

(b)

Figure 6.2: Water droplets on grooved surfaces, showing top-view images of the final droplet shape on two surfaces with identical groove width but different ridge width. At the top a schematic representation of our experimental surfaces is shown, defining relevant parameters $a$ and $b$. (a) Droplet in the Cassie-Baxter state residing on a pattern with groove and ridge widths of $9 \mu \mathrm{m}$ and $6 \mu \mathrm{m}$, respectively. The bright-yellow lines correspond to the empty grooves, while dark stripes correspond to ridges. The dimensions seen through the droplet (which acts as a lens) appear larger; this magnification is even more obvious in case of the Wenzel state. (b) Droplet in the Wenzel state for groove and ridge widths of $9 \mu \mathrm{m}$ and $36 \mu \mathrm{m}$, respectively. The brightness contrast is limited due to the shallow groove depth $(h=2 \mu \mathrm{m})$. The ridge edges appear darker due to scattering of light there. At the bottom, side-view images of the droplets reveal the directionally dependent $\operatorname{CAs}\left(\theta_{\|}\right.$and $\left.\theta_{\perp}\right)$ as well as the diameter of the wetted area in directions perpendicular and parallel (left and right, $N$ and $P$, respectively, as shown in the schematic drawings) to the grooves. 


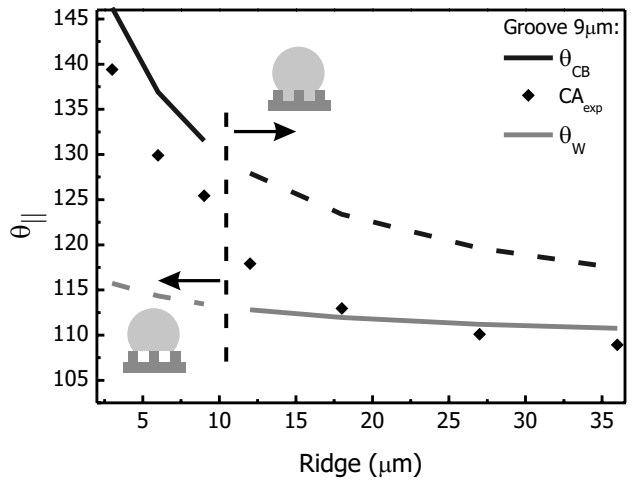

(a)

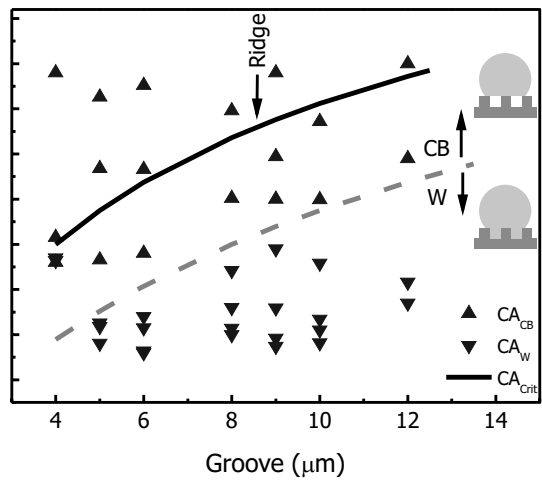

(b)

Figure 6.3: Experimental static water CAs measured in the direction parallel to the grooves $\left(\theta_{\|}\right)$. All patterns have a groove depth of $h=2 \mu \mathrm{m}$. (a) Variation of $\theta_{\|}$ as a function of ridge width for patterns with groove width $b=9 \mu \mathrm{m}$. Solid lines represent theoretical curves for $\theta_{\mathrm{CB}}$ (black line) and $\theta_{\mathrm{W}}$ (gray line). The vertical dotted line indicates the separation between ridge values corresponding to the Cassie-Baxter state $(a<9 \mu \mathrm{m})$ and the Wenzel state $(a>12 \mu \mathrm{m})$. (b) $\theta_{\|}$for all ridge widths studied, plotted as a function of groove width. For every groove width studied, the trend presented in (a) is observed, i.e. patterns with a ridge width smaller than the groove width are in the top part of the plot. Going from top to bottom in each column, the ridge width increases and consequently $\theta_{\|}$decreases. The triangles indicate whether droplets are in the Cassie-Baxter (up triangle) or Wenzel (down triangle) state. The dashed and solid lines are discussed in the text.

CAs $\left(\theta_{\|}\right.$and $\left.\theta_{\perp}\right)$ and diameter lengths of the wetted area in orthogonal directions. The $\theta_{\perp}$ and $N$ appear to have similar values to those of the droplet in the Cassie-Baxter state (compare Fig. 6.2 (a)). However, the $\theta_{\|}$ have markedly smaller values, and the length $P$ is larger as for the droplet in the Cassie-Baxter state.

\subsubsection{Droplet spreading parallel to the grooves}

In Fig. 6.3 the CAs measured in the direction parallel to the grooves $\left(\theta_{\|}\right)$ are presented. To illustrate the general trend, in Fig. 6.3 (a) $\theta_{\|}$for a set of patterns having the same groove width of $9 \mu \mathrm{m}$ are plotted. As the plot reveals, $\theta_{\|}$is maximum for the smallest ridge width of $3 \mu \mathrm{m}$; CA values decrease as the ridge width becomes larger. A similar trend for $\theta_{\|}$is observed for all groove widths we investigated. The two final states observed are 


\subsection{RESULTS}

separated by the dotted line: the line is arbitrarily placed between two experimentally studied ridge widths to indicate where the transition takes place. Furthermore, the $\theta_{\|}$values can be compared to the isotropic $\theta_{\mathrm{CB}}$ and $\theta_{\mathrm{W}}$ calculated by inserting our experimental parameters into eq. 6.1 and eq. 6.2. As observed in the plot, the $\theta_{\mathrm{CB}}$ values agree fairly well with the trend of the $\theta_{\|}$for droplets suspended on the top of the ridges, though theoretical values are slightly higher. In the Wenzel regime, the values of $\theta_{\mathrm{W}}$ are in better agreement with the $\theta_{\|}$despite the fact that we are still dealing with anisotropy for which eq. 6.1 is in principle not valid.

The static $\theta_{\|}$values for all patterns included in this study are plotted in Fig. 6.3 (b). The groove width is presented on the $x$-axis: each vertical column of data points corresponds to a single value for the groove width while the ridge widths are varied, enabling us to present all data in one single graph. The dotted line indicates the separation between two regions: in the upper part of the graph the droplets are in the Cassie-Baxter state, while in the lower part they are in the Wenzel regime. The distinct separation between the two regimes suggests a systematic dependence on the underlying pattern and allows us to use existing equations to account for the results.

In Fig. $6.4(\mathrm{a}, \mathrm{b})$ we plot $\theta_{\|}$as a function of scaling parameters characteristic for each regime. We use the solid fraction $f_{\mathrm{s}}=a /(a+b)$ to plot the data for Cassie-Baxter state, while in the Wenzel state the groove-to-ridge ratio $b / a$ is employed following Patankar's suggestion [112]. Although there is no rigorous theoretical background for using the groove-to-ridge ratio as scaling parameter, trends exhibited by experimental data are most pronounced when the ratio $b / a$ is employed. Equally, theoretical $\theta_{\mathrm{CB}}$ and $\theta_{\mathrm{W}}$ values are plotted, which have been calculated assuming a circular shape of the contact area.

In the Cassie-Baxter regime (Fig. 6.4 (a)) the $\theta_{\|}$values appear to scale to a single line as a function of the solid fraction $f_{\mathrm{s}}$, following the trend predicted by eq. 6.2. Such behavior can be seen as a supplementary confirmation of the regime the droplets are in. The systematically lower experimental values may be due to a slight elongation of the wetted area in the direction of the grooves, resulting in aspect ratios $A R=P / N$ (defined as the ratio of length $P$ and width $N$; see Fig. 6.2) between 1.2 and 1.4 (see Fig. 6.4 (c)). Another reason for observing smaller $\theta_{\|}$values may arise from the way the droplets are deposited on the surface. Previously, it has been observed that releasing droplets from a small altitude leads to lower contact angles due to the kinetic effects [105].

In the Wenzel regime (Fig. 6.4 (b)), the $\theta_{\|}$values appear to scale as a func- 

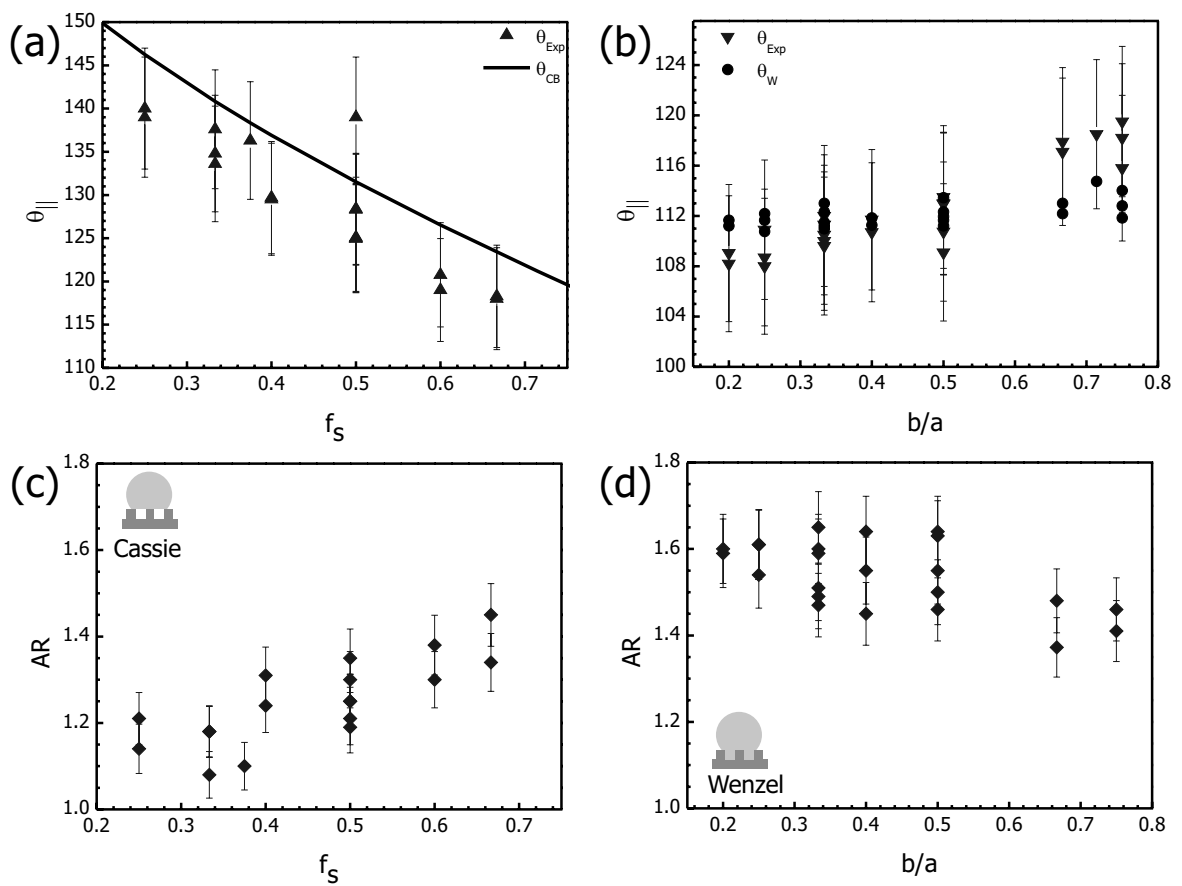

Figure 6.4: Top panels show experimental $\theta_{\|}$values of the droplets. (a) $\theta_{\|}$in the Cassie-Baxter regime as a function of solid fraction $f_{\mathrm{s}}\left(f_{\mathrm{s}}=a /(a+b)\right)$. The theoretical $\theta_{\mathrm{CB}}$ is given by the solid line. (b) $\theta_{\|}$in the Wenzel regime as a function of groove-to-ridge ratio $b / a$. Theoretical $\theta_{\mathrm{W}}$ values are represented by the black dots. Multiple calculated CA values for a single $b / a$ ratio are due to roughness definition $r=(a+b+2 h) /(a+b)$ depending on absolute widths of grooves. In our experiments, several groove/ridge combinations correspond to a single $b / a$ ratio and give slightly different $r$ values. We choose to plot $\theta_{\mathrm{W}}$ as function of $b / a$ ratio and not the scaling parameter of the Wenzel regime, i.e. roughness $r$, as plotting CAs versus roughness does not exhibit a clear trend. Droplet aspect ratios are shown in the bottom panels for the Cassie-Baxter (c) and Wenzel (d) regimes.

tion of the groove-to-ridge ratio $b / a$. Despite the fact that the roughness $r$ is a scaling parameter for the Wenzel regime, using it for plotting masks the trend observed in the experimental data. Both $\theta_{\|}$and $\theta_{\mathrm{W}}$ values increase with $b / a$ ratio, though the trends are different. For $\theta_{\|}$, the patterns with low $b / a$ ratios exhibit the lowest CAs with values similar to the $\theta_{\mathrm{St}}$ on the flat surface. As the $b / a$ ratio, and thus the number of grooves under the droplet increases, so does $\theta_{\|}$, until it reaches a maximum value of $120^{\circ}$. The trend predicted by the Wenzel equation (eq. 6.1), shown by the black 


\subsection{RESULTS}

dots in Fig. 6.4 (b), also exhibits a rise of $\theta_{\mathrm{W}}$ with $b / a$, but less pronounced as compared to the experimental data. The reason for the relatively small variation of calculated $\theta_{\mathrm{W}}$-values lies in the shallow depth of the grooves $(2 \mu \mathrm{m})$. For ratios $b / a$ below approximately 0.3 , i.e. patterns having large ridge widths compared to the grooves, the $\theta_{\|}$are smaller than $\theta_{\mathrm{W}}$. For the $b / a$ ratio around 0.5 , theory and experiment exhibit similar values. Once the ratios exceed 0.6 , i.e. when the ridge width approaches the groove width, the calculated $\theta_{\mathrm{W}}$ values are smaller than $\theta_{\|}$.

Furthermore, the values of $\theta_{\|}$measured in the Wenzel regime for $b / a>$ 0.6 are similar to those exhibited in the Cassie-Baxter regime for solid fractions $f_{\mathrm{s}}$ between 0.6 and 0.7 , corresponding to situations in which the ridge is slightly larger than the groove, as can be seen from Fig. 6.4 (a). Such elevated $\theta_{\|}$values in the Wenzel regime may be explained based on the observation that, despite the deposition of droplets via gentle lowering (characteristic of liquid filling the underlying structures), they tend first to reside for a short period of time in the partial Cassie-Baxter state (see also Fig. 6.6). Spontaneous collapse into the energetically more favorable Wenzel state always occurs within seconds. More details pertaining to the kinetics of droplet deposition are presently under investigation and are considered beyond the scope of this chapter.

To study the influence of the anisotropic pattern on the final shape and to quantify the distortion from a spherical shape, the aspect ratios for droplets in both regimes are plotted in Fig. 6.4 (c) and Fig. 6.4 (d). To identify trends in the experimental data, the $A R$ values are plotted as a function of grooveto-ridge ratio $b / a$ in the Wenzel regime, while in the Cassie-Baxter regime the solid fraction $f_{\mathrm{s}}$ is used to present the data.

In the Cassie-Baxter regime, the final shape of the droplets shows a relatively small deviation from a spherical geometry due to the underlying anisotropic pattern. For small $f_{\mathrm{s}}$, i.e. when the droplet is primarily suspended over the air pockets between the ridges, the final shape is almost spherical, showing only a very slight elongation in the direction of the grooves $(A R \approx 1)$. As the solid part in contact with the droplet increases, the influence of the pattern on the final shape becomes more pronounced as the $A R$ increases to reach values of 1.4 for $f_{\mathrm{s}}>0.5$. Furthermore, both diameters parallel $P$ and perpendicular $N$ to the grooves become larger as $f_{\mathrm{s}}$ increases.

The highest experimental $A R$ values are observed in the Wenzel regime, reaching maximum values of approximately 1.6 (Fig. 6.4 (d)). Considering that the $\theta_{\|}$values for these substrates are nearly equal to those on a flat surface (Fig. 6.4 (b)), it seems that the elongation cancels an increase of $\theta_{\|}$ due to the grooves beneath the droplet. As the groove-to-ridge ratio $b / a$ 

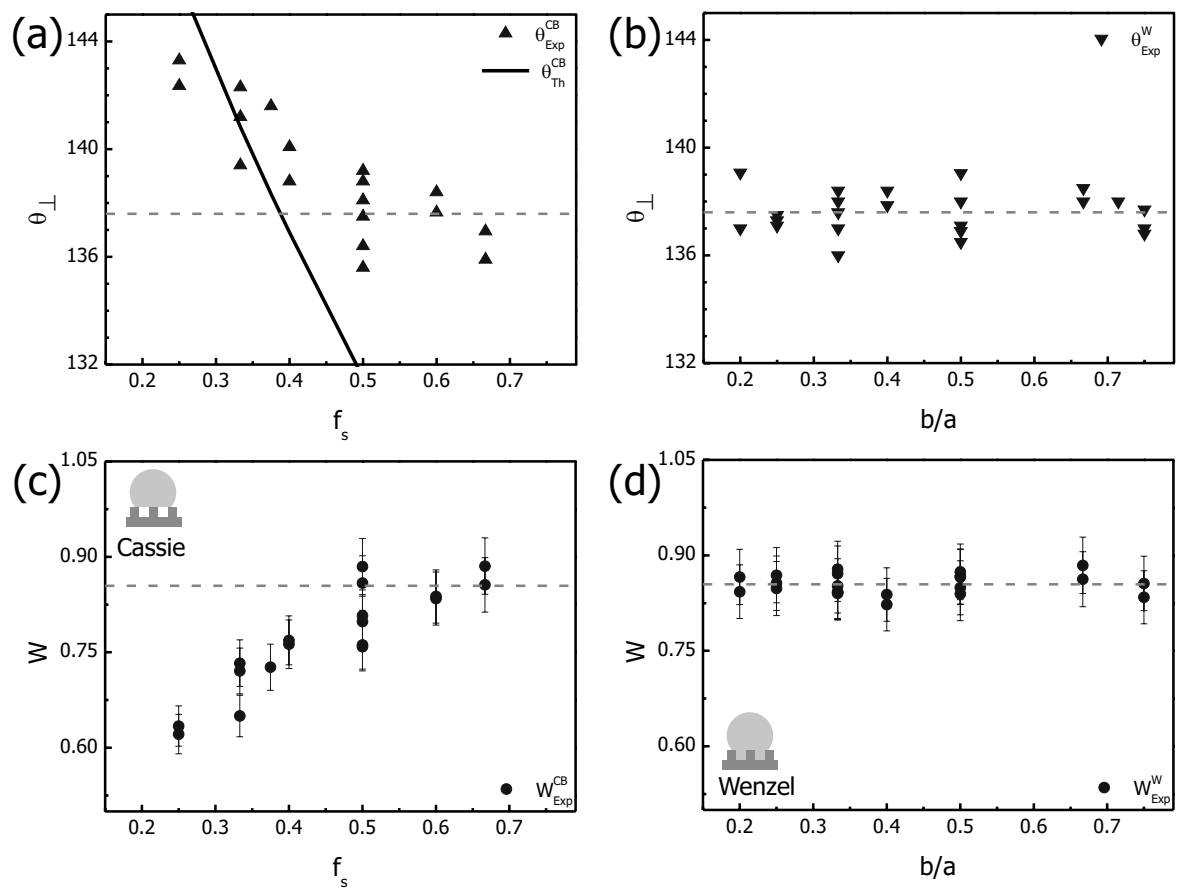

Figure 6.5: (Color online) Top panels show experimental $\theta_{\perp}$ values of the droplets. (a) $\theta_{\perp}$ in the Cassie-Baxter regime as a function of solid fraction $\left.f_{\mathrm{s}}\left(f_{\mathrm{s}}=a /(a+b)\right)\right)$. Theoretical $\theta_{\mathrm{CB}}$ is plotted as solid line to show that only part of the data seems to follow the model predictions. (b) $\theta_{\perp}$ in the Wenzel regime as a function of groove-to-ridge ratio $b / a$. The values scatter around $137.6^{\circ}$ for all patterns studied. Bottom panels show the evolution of the wetted area diameter measured perpendicular to the stripes $N$ for the Cassie-Baxter (c) and Wenzel (d) regimes.

increases, the $A R$ decreases to finally scatter around 1.4. Interestingly, all droplets in Wenzel regime have $N$ values scattering around $0.85 \mathrm{~mm}$; the observed trend for $A R$ is governed solely by the variation of the droplet length $P$ (see Fig. 6.5).

\subsubsection{Droplet spreading perpendicular to the grooves}

The $\theta_{\perp}$ and $N$ trends allow a better assessment of equilibrium shapes. Other than presenting a very different behavior compared to the results in the direction parallel to the grooves, they provide a more profound indication of similarities between the regimes (in the case of $f_{\mathrm{s}}>0.5$ and $b / a>0.5$ ). In Fig. 6.5 the apparent macroscopic CAs $\theta_{\perp}$ in the direction perpendicular to 


\subsection{RESULTS}

the grooves (Fig. $6.5(\mathrm{a}, \mathrm{b})$ ) and the diameter $N$ of the wetted area (Fig. 6.5 $(c, d))$ are plotted as a function of $f_{\mathrm{s}}$ (Cassie-Baxter regime) and $b / a$ ratio (Wenzel regime).

In the Wenzel regime $\theta_{\perp}$ (Fig. 6.5 (b)) scatters around a mean value of $137.6^{\circ}$ for all patterns considered in this work; equally, the droplet diameters $N$ scatter around $0.85 \mathrm{~mm}$ (Fig. 6.5 (d)). Such independence of the final dimensions on the underlying pattern, especially for large $b / a$ ratio, would suggest a similar spreading mechanism close to reaching the static shape. As mentioned previously, for $b / a>0.5$ parts of the droplet remain suspended and the grooves are filled within seconds after deposition. Given the same CA and $N$ values, it would mean that the filled or empty state of the grooves away from the border of the droplet has little influence on the static values in the direction perpendicular to the grooves.

In the Cassie-Baxter regime (Fig. $6.5(\mathrm{a}, \mathrm{c})$ ) for $f_{\mathrm{s}}<0.5$, the $\theta_{\perp}$ decrease as $f_{\mathrm{s}}$ increases as well as exhibits values, which are similar to $\theta_{\|}$; this is to be expected since the droplets have a spherical geometry with low $A R$ values close to 1.2 (see Fig. $6.4(\mathrm{a}, \mathrm{c})$ ). The diameter $N$ of the wetted area also becomes larger as $f_{\mathrm{s}}$ increases in agreement with the variation of CA values. In short, for $f_{\mathrm{s}}<0.5$ the behavior expected for droplets in CassieBaxter regime is observed.

Moreover, such limited influence of the underlying pattern with small $f_{\mathrm{s}}$ on the final shape in case of the Cassie-Baxter final state is of interest by itself. Previously reported spherically shaped droplets on anisotropic microscaled [118] or grooved [124] surfaces are attributed to high roughness of surfaces on both microscopic and nanoscopic scales, which is not the case for our experimental surfaces. For droplets suspended on the smooth ridge tops, the distortion of the contact area that follows the underlying structure is observed. [84, 122] Moreover, the suspended state appears to be stable and the spontaneous transition into the energetically more favorable Wenzel state does not take place. Given the shallowness of the structures, the stability may be attributed to the pinning of the contact line on the edges of the ridges. [125]

However, for $f_{\mathrm{s}}>0.5$ the values do not show any dependence on the underlying pattern: $\theta_{\perp}$ scatters around a value of $137.6^{\circ}$ and $N$ scatters around $0.85 \mathrm{~mm}$. The same behavior as well as similar values are observed for the droplets in the Wenzel regime. Studying of the top view images of droplets reveals no filled grooves at the regions close to the edge of the droplets. Comparing experimental results for different groove widths reveals identical values of $\theta_{\perp}$ and $N$ once the ridge width exceeds $8 \mu \mathrm{m}$, suggesting that the width of the grooves has very limited or no influence at 
all. Moreover, combined with the similarity to the results for droplets in the Wenzel regime on patterns with $b / a>0.5$, where the grooves near the edge of the droplet are filled, it can be reasoned that the filled or empty state of grooves is not the parameter that influences the static shape in this case.

\subsection{Discussion}

In the previous section we have described our observations pertaining to droplets on morphologically stripe-patterned surfaces. Depending on the relative ridge and groove widths, droplets either adopt the Cassie-Baxter or Wenzel state. In this section we first discuss the observations in relation to energetic considerations. Next, we focus on the main issue of the present chapter, i.e. the transition from the metastable Cassie-Baxter to Wenzel state as a function of ridge width.

\subsubsection{Final state of droplets: theoretical predictions}

As described in the introduction, on low surface energy substrates in which liquid has the static $\mathrm{CAs} \theta_{\mathrm{St}}>90^{\circ}$ generally two states are observed when the surface exhibits roughness: Wenzel or Cassie-Baxter state. Using energy arguments, one can calculate the apparent CAs in both regimes for a given rough surface. Direct comparison of the calculated $\theta_{\mathrm{CB}}$ and $\theta_{\mathrm{W}}$ for a given surface allows evaluation of the relative stability of the two states: the regime exhibiting a smaller macroscopic apparent CA corresponds to a lower energy state and should be preferentially adopted by the liquid. Plotting the cosines of both $\theta_{\mathrm{CB}}$ and $\theta_{\mathrm{W}}$ as a function of substrate roughness presents a simple way to identify whether either the CassieBaxter or the Wenzel state corresponds to the thermodynamic equilibrium as well as give an indication of the energy barrier separating the two states. Generally, if droplets end up in the 'fakir' state on a surface where the Wenzel regime is energetically more favorable, the droplets are said to be in metastable Cassie-Baxter state. Furthermore, the intersection point between the two regimes, corresponding to surface structures when both states have the same energy, and consequently, the same apparent CA, can be calculated.

A relatively simple way to predict apriori which state should be adopted on a given hydrophobic structured surface was suggested by D. Quéré [104, 125]. If the roughness $r$ and solid fraction $f_{\mathrm{s}}$ are known, the critical CA $\theta_{\mathrm{Cr}}$ 


\subsection{DISCUSSION}

for a flat surface with similar properties can be calculated:

$$
\cos \theta_{\mathrm{Cr}}=\frac{1-f_{\mathrm{s}}}{r-f_{\mathrm{s}}}
$$

Comparing our experimental $\theta_{\mathrm{St}}$ with calculated $\theta_{\mathrm{Cr}}$ estimates which of the two is more likely to be adopted. If $\theta_{\mathrm{St}}$ on the flat surface is smaller than $\theta_{\mathrm{Cr}}\left(\theta_{\mathrm{St}}<\theta_{\mathrm{Cr}}\right)$, than the Wenzel state is energetically more favorable. Otherwise, the Cassie-Baxter or 'fakir' state corresponds to the situation with minimum energy. In our case for grooved surfaces the roughness $r$ is given by [102]:

$$
r=\frac{a+b+2 h}{a+b}
$$

where $h$ is the depth of the grooves, fixed to $2 \mu \mathrm{m}$. Inserting this, together with $f_{\mathrm{s}}=a /(a+b)$, into 6.3, we obtain the following expression for $\theta_{\mathrm{Cr}}$ :

$$
\cos \theta_{\mathrm{Cr}}=\frac{-1}{1+\frac{2 h}{b}}
$$

We find that the critical angle is independent of the ridge width $a$ but only depends on the groove width $b$ and the ridge height $h$. A similar dependence solely on pitch width and height of the structures has been described for other geometries. [105, 126, 127]

The resulting values for $\theta_{\mathrm{Cr}}$ are represented in Fig. 6.3 (b) by the solid black line, changing from $120^{\circ}$ for a groove width of $4 \mu \mathrm{m}$ to $138.6^{\circ}$ for a groove width of $12 \mu \mathrm{m}$. On the unpatterned PFDTS treated silicon wafer we measure the $\theta_{\mathrm{St}}=109^{\circ}$ what makes $\theta_{\mathrm{St}}<\theta_{\mathrm{Cr}}$ for all studied patterns. Consequently, the Cassie-Baxter state does not correspond to the minimum energy situation for all surfaces considered in our present work. Most likely, the reason why we indeed observe this metastable state on a number of patterns is related to the way the droplets are deposited on the surface, i.e. by dropping them from a certain height $[104,105]$. However, that does not account for the Cassie-Baxter state stability on our experimental surfaces.

Further, the calculated $\theta_{\mathrm{Cr}}$ (Fig. 6.3 (b), solid line) for all groove widths appear to follow a similar trend as, though are systematically larger than the border region between patterns on which the metastable Cassie-Baxter state is observed and patterns where droplets are in the Wenzel state. Moreover, calculations for the critical $\theta_{\mathrm{Cr}}$ agree well with the experimentally observed transition from one regime to the other when the height-to-groove width ratio in the denominator in eq. 6.5 is multiplied by a factor of 2 (Fig. 6.3 (b), gray dashed line). This correction holds for all experimental 


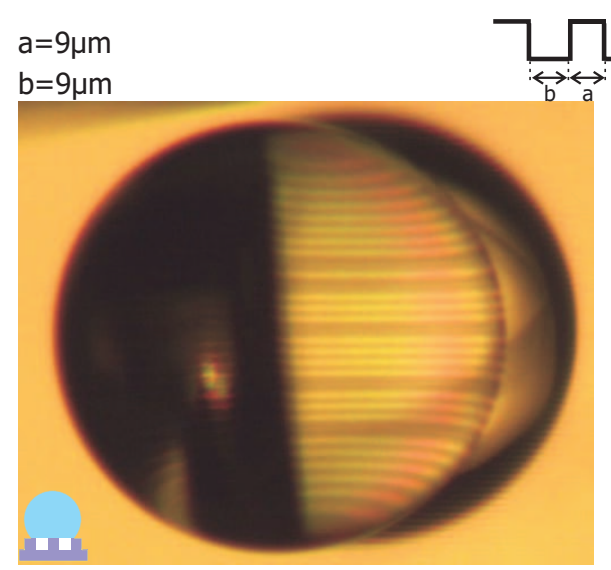

(a)

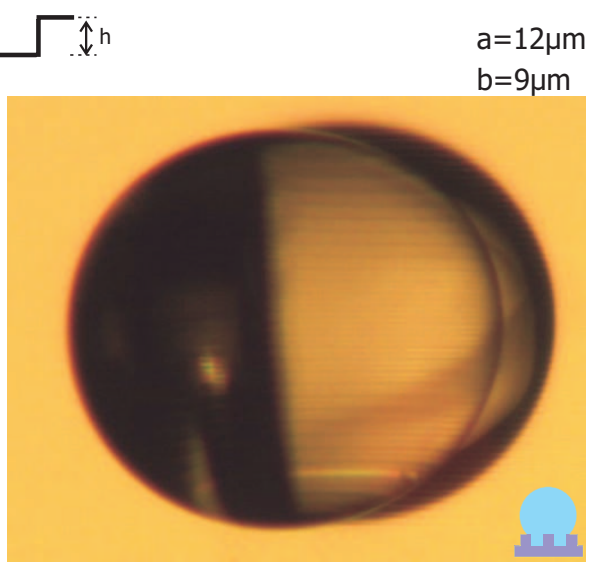

(b)

Figure 6.6: Snapshots of droplets observed on surfaces with (a) $f_{\mathrm{s}}=0.5$ (CassieBaxter regime) and (b) $b / a=0.75$ (Wenzel regime). In both regimes the droplets appear to have almost spherical shapes irrespective of the way the droplets were deposited on the surface.

sets studied, though the origin of this multiplication factor is unclear. Summarizing, the aforementioned model, which has been nicely described by Bico et al. [111] seems to be in qualitative agreement with our observations; why this is the case for our anisotropic grooved surfaces is unclear.

\subsubsection{Transition from metastable Cassie-Baxter to stable Wenzel}

Returning to the data presented in Fig. 6.5, on a fraction of the patterns where the groove and ridge widths are equal or very close (i.e. in the case of $f_{\mathrm{s}}>0.5$ and $b / a>0.5$ ), both states seem to exhibit similar values for the CAs and diameters of the wetted area. As an illustration in Fig. 6.6 two snapshots are shown of droplets, one being mostly suspended on the grooves (Fig. 6.6 (a)) while another almost completely filling the underlying structures (Fig. 6.6 (b)). The droplets ending up in the Wenzel state are initially in a partial Cassie-Baxter state leaving various amount of grooves under the droplet not filled, but almost immediately collapse into the Wenzel state. The same happens if the droplets are dropped from a low height.

It appears that the patterns considered here are near the critical point where both states have the same energies, and, supposedly, the transition between states occurs. On the patterns where the Cassie-Baxter state is observed, the energy barrier is just high enough to hinder the transition, 


\subsection{DISCUSSION}

while for the other patterns the transition barrier to the Wenzel state is low, allowing the droplets to go easily into the energetically more favorable situation from the metastable suspended state they are deposited in. Moreover, theoretically estimated critical points between filled and suspended states do not correlate with the experimentally observed transition, which possibly is the result of the anisotropy of the patterns.

To understand the liquid behaviour on these patterns near the critical point, we can use the existing knowledge of contact line advancing in both regimes. Summarizing, in case of the Wenzel state, prior to filling of the next groove the advancing CA condition at the edge of the ridge has to be met. Once the dynamic CA at the edge reaches a value lower than the advancing CA, the droplet remains pinned and spreading in the direction perpendicular to grooves stops $[29,41]$. In the Cassie-Baxter state, the advancing edge of the droplet 'falls' until it reaches the top of the neighboring ridge at the location corresponding to the area with the largest curvature of the droplet. Subsequently, it will advance over this next ridge if the condition of the advancing CA for a flat surface is met [114]. In both cases, the contact line will be pinned on the edge of the ridge before the next groove is bridged.

Assuming that both suspended and filled states have very similar energies, and consequently, exhibit similar values for macroscopic $\theta_{\mathrm{CB}}$ or $\theta_{\mathrm{W}}$ values, it is safe to suppose that the microscopic dynamic angles reach values prohibiting further advancing for similar macroscopic lengths. However, the width of the ridge should be large enough (in our case $>8 \mu \mathrm{m}$ ), for similar $N$ and $\theta_{\perp}$ in both regimes irrespective of the last groove 'bridged' by the droplet being filled with liquid or whether the droplet remains suspended above it.

Considering the similar values for $P$ and $\theta_{\|}$observed on these surfaces (Fig. 6.4), the explanation most likely lies in the fact that droplets in the Wenzel state have not achieved their minimum energy shape. In the suspended state, the advancing contact line advances over the composite surface of air and hydrophobic solid, encountering a low energy barrier, resulting in static $\theta_{\|}$values close to the ones predicted by Cassie-Baxter equation. For the filled state, the complete filling of the underlying structures occurs at later stages, the first shape being to a certain degree defined by spreading over the composite surface. Once the entire transition to the Wenzel state has taken place, it is possible that the microscopic conditions for the advancing CA are no longer fulfilled and further filling of grooves to achieve a lower macroscopic apparent CA is not possible. That would explain the experimental $\theta_{\|}$exhibiting larger values as compared to the 
ones estimated by the Wenzel equation.

\subsection{Conclusions}

We have studied the equilibrium droplet behavior on anisotropic shallow grooved surfaces. The groove geometry creates two orthogonal spreading directions with different properties, resulting in elongated droplets with directionally dependent properties. From the experimental results, we find that two states are reproducibly observed on the studied anisotropic surfaces: (i) metastable Cassie-Baxter and (ii) Wenzel states, the latter corresponding to the thermodynamic equilibrium on our surfaces. Comparison between experimental $\theta_{\|}$and $\theta_{\mathrm{CB}}$ and $\theta_{\mathrm{W}}$ predicted by theoretical equations reveals a similar trend but systematically lower values for droplets in the Cassie-Baxter regime. In the Wenzel regime, there is no agreement in trend. The elongation in the direction of the grooves is more pronounced for the Wenzel regime, while in the Cassie-Baxter regime the influence of the underlying pattern on the deviation of the droplet shape from a spherical geometry is much less pronounced. The energy barrier between the suspended state as compared to a complete filling of the underlying structures is low on a fraction of patterns, resulting in spontaneous transition from the Cassie-Baxter to the Wenzel state. Moreover, elevated values for $\theta_{\|}$in the Wenzel state are observed. Finally, calculating the stability condition for both thermodynamically stable Cassie-Baxter and Wenzel states on surfaces with well-defined structures, we find that calculated $\theta_{\mathrm{Cr}}$ values seem to agree with the transition trend between patterns observed for apparent $\theta_{\mathrm{CB}}$ and $\theta_{\mathrm{W}}$. 




\section{Bibliography}

[1] J. Zeleny, The electrical discharge from liquid points, and a hydrostatic method of measuring the electric intensity at their surfaces, Phys.Rev. 3, 69 (1914).

[2] H. P. Le, Progress and trends in ink-jet printing technology, J. Imaging Sci. Technol. 42, 49 (1998).

[3] H. Wijshoff, The dynamics of the piezo inkjet printhead operation, Phys. Rep. 491, 77 (2010).

[4] C. Griggs and J. Sumerel, Opportunities for inkjet printing in industrial applications, Industrial+Specialty Printing (Apr 2010).

[5] S. M. Bidoki, J. Nouri, and A. A. Heidari, Inkjet deposited circuit components, J. Micromech. Microeng. 20, 7 (2010).

[6] J. Y. Hwang and L. C. Chien, Emerging Liquid Crystal Technologies V (Spie-Int Soc Optical Engineering, Bellingham, 2010), Vol. 7618.

[7] D. Kim, Y. Jeong, K. Song, S. K. Park, G. Z. Cao, and J. Moon, Inkjetprinted zinc tin oxide thin-film transistor, Langmuir 25, 11149 (2009).

[8] D. Y. Shin, Fabrication of an inkjet-printed seed pattern with silver nanoparticulate ink on a textured silicon solar cell wafer, J. Micromech. Microeng. 20, 10 (2010).

[9] S. I. E. Lin, Study and implementation of a hybrid diffractive/refractive lens and a color inkjet head on high density data storage, Appl. Optics 50, 1091 (2011).

[10] J. C. Carter, R. M. Alvis, S. B. Brown, K. C. Langry, T. S. Wilson, M. T. McBride, M. L. Myrick, W. R. Cox, M. E. Grove, and B. W. Colston, Fabricating optical fiber imaging sensors using inkjet printing technology: A pH sensor proof-of-concept, Biosens. Bioelectron. 21, 1359 (2006).

[11] G. Arrabito and B. Pignataro, Inkjet printing methodologies for drug screening, Anal. Chem. 82, 3104 (2010).

[12] MEMS inkjet printheads: new developments for growing industrial applications, Micronews (Jan 2009).

[13] MEMS : status of the MEMS industry, Micronews (Sep 2010). 
[14] J. de Jong, H. Reinten, H. Wijshoff, M. van den Berg, K. Delescen, R. van Dongen, F. Mugele, M. Versluis, and D. Lohse, Marangoni flow on an inkjet nozzle plate, Appl. Phys. Lett. 91, 3 (2007).

[15] B. Beulen, J. de Jong, H. Reinten, M. van den Berg, H. Wijshoff, and R. van Dongen, Flows on the nozzle plate of an inkjet printhead, Exp. Fluids 42, 217 (2007).

[16] J. de Jong, G. de Bruin, H. Reinten, M. van den Berg, H. Wijshoff, M. Versluis, and D. Lohse, Air entrapment in piezo-driven inkjet printheads, J. Acoust. Soc. Am. 120, 1257 (2006).

[17] Dimatix, Wetting of nozzle plate imaged by JetXpert drop watcher system, (Feb 2011).

[18] H. Kusumaatmaja and J. M. Yeomans, Modeling contact angle hysteresis on chemically patterned and superhydrophobic surfaces, Langmuir 23, 6019 (2007).

[19] A. A. Darhuber, S. M. Troian, S. M. Miller, and S. Wagner, Morphology of liquid microstructures on chemically patterned surfaces, J. Appl. Phys. 87, 7768 (2000).

[20] H. Gau, S. Herminghaus, P. Lenz, and R. Lipowsky, Liquid morphologies on structured surfaces: from microchannels to microchips, Science 283, 46 (1999).

[21] C. Bauer and S. Dietrich, Phase diagram for morphological transitions of wetting films on chemically structured substrates, Phys. Rev. E 61, 1664 (2000).

[22] M. Brinkmann and R. Lipowsky, Wetting morphologies on substrates with striped surface domains, J. Appl. Phys. 92, 4296 (2002).

[23] X. M. Li, D. Reinhoudt, and M. Crego-Calama, What do we need for a superhydrophobic surface? A review on the recent progress in the preparation of superhydrophobic surfaces, Chem. Soc. Rev. 36, 1350 (2007).

[24] L. Gao, A. Y. Fadeev, and T. McCarthy, Superhydrophobicity and contact line issues, MRS Bull. 33, 747 (2008).

[25] P. Lenz and R. Lipowsky, Morphological transitions of wetting layers on structured surfaces, Phys. Rev. Lett. 80, 1920 (1998). 


\section{BIBLIOGRAPHY}

[26] C. Neinhuis and W. Barthlott, Characterization and distribution of water-repellent, self-cleaning plant surfaces, Ann. Bot. (Lond) 79, 667 (1997).

[27] P. Roach, N. J. Shirtcliffe, and M. Newton, Progress in superhydrophobic surface development, Soft Matter 4, 224 (2008).

[28] R. Dettre and R. Johnson, Wettability (CRC Press, New York, 1993), pp. 1-73.

[29] H. Kusumaatmaja, R. J. Vrancken, C. W. M. Bastiaansen, and J. M. Yeomans, Anisotropic drop morphologies on corrugated surfaces, Langmuir 24, 7299 (2008).

[30] W. Li, G. Fang, Y. Li, and G. Qiao, Anisotropic wetting behavior arising from superhydrophobic surfaces: parallel grooved structure, J. Phys. Chem. B 112, 7234 (2008).

[31] D. Xia and S. Brueck, Strongly anisotropic wetting on one-dimensional nanopatterned surfaces, Nano Lett. 8, 2819 (2008).

[32] J. Chung, J. Youngblood, and C. Stafford, Anisotropic wetting on tunable micro-wrinkled surfaces, Soft. Matt. 3, 1163 (2007).

[33] J. C. Love, L. Estroff, J. Kriebel, R. Nuzzo, and G. Whitesides, SelfAssembled monolayers of thiolates on metals as a form of nanotechnology, Chem. Rev. 105, 1103 (2005).

[34] H. Rathgen, Ph.D. thesis, University of Twente, 2008.

[35] M. Iwamatsu, Contact angle hysteresis of cylindrical drops on chemically heterogeneous striped surfaces, J. Colloid Interface Sci. 297, 772 (2006).

[36] M. Iwamatsu, The validity of Cassie's law: a simple exercise using a simplified model, J. Colloid Interface Sci. 294, 176 (2006).

[37] T. Pompe and S. Herminghaus, Three-phase contact line energetics from nanoscale liquid surface topographies, Phys. Rev. Lett. 85, 1930 (2000).

[38] A. B. D. Cassie and S. Baxter, Wettability of porous surfaces, Trans. Farad. Soc. 40, 546 (1944).

[39] M. Gleiche, L. Chi, E. Gedig, and H. Fuchs, Anisotropic contact-angle hysteresis of chemically nanostructured surfaces, ChemPhysChem 2, 187 (2001). 


\section{BIBLIOGRAPHY}

[40] A. Marmur, Soft contact: measurement and interpretation of contact angles, Soft Matter 2, 12 (2006).

[41] P. G. de Gennes, Wetting: statics and dynamics, Rev. Mod. Phys. 57, 827 (1985).

[42] M. Morita, T. Koga, H. Otsuka, and A. Takahara, Macroscopic-wetting anisotropy on the line-patterned surface of fluoroalkylsilane monolayers, Langmuir 21, 911 (2005).

[43] J. Léopoldès and D. G. Bucknall, Droplet spreading on microstriped surfaces, J. Phys. Chem. B 109, 8973 (2005).

[44] O. Bliznyuk, H. P. Jansen, E. S. Kooij, and B. Poelsema, Initial spreading kinetics of high-viscosity droplets on anisotropic surfaces, Langmuir 26, 6328 (2010).

[45] P. Poole, F. Sciortino, T. Grande, H. Stanley, and C. Angell, Effect of hydrogen bonds on the thermodynamic behavior of liquid water, Phys. Rev. Lett. 73, 1632 (1994).

[46] A. Luzar and D. Chandler, Hydrogen-bond kinetics in liquid water, Nature 379, 55 (1996).

[47] L. J. Root and B. J. Berne, Effect of pressure on hydrogen bonding in glycerol: A molecular dynamics investigation, J. Chem. Phys. 107, 4350 (1997).

[48] R. Chelli, P. Procacci, G. Cardini, and S. Califano, Glycerol condensed phases Part II. A molecular dynamics study of the conformational structure and hydrogen bonding, Phys. Chem. Chem. Phys. 1, 879 (1999).

[49] V. Bergeron, D. Bonn, J. Y. Martin, and L. Vovelle, Controlling droplet deposition with polymer additives, Nature 405, 772 (2000).

[50] D. Bonn, J. Eggers, J. Indekeu, J. Meunier, and E. Rolley, Wetting and spreading, Rev. Mod. Phys. 81, 739 (2009).

[51] D. Lasne, A. Maali, Y. Amarouchene, L. Cognet, B. Lounis, and H. Kellay, Velocity profiles of water flowing past solid glass surfaces using fluorescent nanoparticles and molecules as velocity probes, Phys. Rev. Lett. 100, 4 (2008). 


\section{BIBLIOGRAPHY}

[52] D. Palms, C. Priest, R. Sedev, J. Ralston, and G. Wegner, Directed crystallisation of zinc oxide on patterned surfaces, J. Colloid Interface Sci. 303, 333 (2006).

[53] A. L. Biance, C. Clanet, and D. Quéré, First steps in the spreading of a liquid droplet, Phys. Rev. E 69, 4 (2004).

[54] R. Kannan and D. Sivakumar, Drop impact process on a hydrophobic grooved surface, Colloid Surf. A 317, 694 (2008).

[55] Y. D. Shikhmurzaev, Moving contact lines in liquid/liquid/solid systems, J. Fluid Mech. 334, 211 (1997).

[56] J. Drelich and D. Chibowska, Spreading kinetics of water drops on selfassembled monolayers of thiols: Significance of inertial effects, Langmuir 21, 7733 (2005).

[57] B. Lavi and A. Marmur, The exponential power law: partial wetting kinetics and dynamic contact angles, Colloid Surf. A 250, 409 (2004).

[58] L. H. Tanner, Spreading of silicon oil drops on horizontal surfaces, J. Phys. D 12, 1473 (1979).

[59] A. M. Cazabat and M. A. C. Stuart, Dynamics of wetting - effects of surface roughness, J. Phys. Chem. 90, 5845 (1986).

[60] J. Lopez, C. A. Miller, and E. Ruckenstein, Spreading kinetics of liquiddrops on solids, J. Colloid Interface Sci. 56, 460 (1976).

[61] C. Huh and L. E. Scriven, Hydrodynamic model of steady movement of a solid/liquid/fluid contact line, J. Colloid Interface Sci. 35, 85 (1971).

[62] J. Eggers, J. R. Lister, and H. A. Stone, Coalescence of liquid drops, J. Fluid Mech. 401, 293 (1999).

[63] J. C. Bird, S. Mandre, and H. A. Stone, Short-time dynamics of partial wetting, Phys. Rev. Lett. 100, 4 (2008).

[64] H. Ding and P. D. M. Spelt, Inertial effects in droplet spreading: a comparison between diffuse-interface and level-set simulations, J. Fluid Mech. 576, 287 (2007).

[65] J. P. Youngblood and T. J. McCarthy, Ultrahydrophobic polymer surfaces prepared by simultaneous ablation of polypropylene and sputtering of poly(tetrafluoroethylene) using radio frequency plasma, Macromolecules 32, 6800 (1999). 
[66] L. E. Scriven and C. V. Sternling, The Marangoni effects, Nature 187, 186 (1960).

[67] J. Thomson, On certain curious motions observable at the surfaces of wine and other alcoholic liquors, Phil. Mag. 10, 330 (1855).

[68] C. Marangoni, Ueber die ausbreitung der tropfen einer flussigkeit auf der oberflache einer anderen, Ann. Phys. 219, 337 (1871).

[69] D. T. Wasan, A. D. Nikolov, and H. Brenner, Fluid dynamics - droplets speeding on surfaces, Science 291, 605 (2001).

[70] A. A. Darhuber, J. P. Valentino, S. M. Troian, and S. Wagner, Thermocapillary actuation of droplets on chemically patterned surfaces by programmable microheater arrays, J. Microelectromech. Syst. 12, 873 (2003).

[71] Y. T. Tseng, F. G. Tseng, Y. F. Chen, and C. C. Cheng, Fundamental studies on micro-droplet movement by Marangoni and capillary effects, Sens. Actuator A-Phys. 114, 292 (2004).

[72] L. W. Schwartz, R. V. Roy, R. R. Eley, and H. M. Princen, Surfactantdriven motion and splitting of droplets on a substrate, J. Eng. Math. 50, 157 (2004).

[73] H. P. Greenspan, On the motion of a small viscous droplet that wets a surface, J. Fluid Mech. 84, 125 (1978).

[74] F. Brochard, Motion of droplets on solid-surfaces induced by chemical or thermal-gradients, Langmuir 5, 432 (1989).

[75] R. Tadmor, Marangoni flow revisited, J. Colloid Interface Sci. 332, 451 (2009).

[76] M. K. Chaudhury and G. M. Whitesides, How to make water run uphill, Science 256, 1539 (1992).

[77] X. Zhu, H. Wang, Q. Liao, Y. D. Ding, and Y. B. Gu, Experiments and analysis on self-motion behaviors of liquid droplets on gradient surfaces, Exp. Therm. Fluid Sci. 33, 947 (2009).

[78] Y. Ito, M. Heydari, A. Hashimoto, T. Konno, A. Hirasawa, S. Hori, K. Kurita, and A. Nakajima, The movement of a water droplet on a gradient surface prepared by photodegradation, Langmuir 23, 1845 (2006). 


\section{BIBLIOGRAPHY}

[79] D. Hong, W. Cho, B. Kong, and I. Choi, Water-collecting capability of radial-wettability gradient surfaces generated by controlled surface reactions, Langmuir 26, 15080 (2010).

[80] J. B. Brzoska, F. Brochardwyart, and F. Rondelez, Motions of droplets on hydrophobic model surfaces induced by thermal-gradients, Langmuir $\mathbf{9}$, 2220 (1993).

[81] S. Daniel and M. K. Chaudhury, Rectified motion of liquid drops on gradient surfaces induced by vibration, Langmuir 18, 3404 (2002).

[82] R. S. Subramanian, N. Moumen, and J. B. McLaughlin, Motion of a drop on a solid surface due to a wettability gradient, Langmuir 21, 11844 (2005).

[83] H. Suda and S. Yamada, Force measurements for the movement of a water drop on a surface with a surface tension gradient, Langmuir 19, 529 (2003).

[84] J. Z. Chen, S. M. Troian, A. A. Darhuber, and S. Wagner, Effect of contact angle hysteresis on thermocapillary droplet actuation, J. Appl. Phys. 97, 9 (2005).

[85] O. Bliznyuk, E. Vereshchagina, E. Kooij, and B. Poelsema, Scaling of anisotropic droplet shapes on chemically stripe-patterned surfaces, Phys. Rev. E 79, 041601 (2009).

[86] C. Williams, Ink-jet printers go beyond paper, Phys. World 19, 24 (2006).

[87] C. T. Chen, K. H. Wu, C. F. Lu, and F. Shieh, An inkjet printed stripetype color filter of liquid crystal display, J. Micromech. Microeng. 20, 11 (2010).

[88] B. Fousseret, M. Mougenot, F. Rossignol, J. F. Baumard, B. Soulestin, C. Boissiere, F. Ribot, D. Jalabert, C. Carrion, C. Sanchez, and M. Lejeune, Inkjet-printing-engineered functional microdot arrays made of mesoporous hybrid organosilicas, Chem. Mat. 22, 3875 (2010).

[89] R. Beccherelli, E. Zampetti, S. Pantalei, M. Bernabei, and K. C. Persaud, Design of a very large chemical sensor system for mimicking biological olfaction, Sens. Actuator B-Chem. 146, 446 (2010).

[90] Z. Y. Cheng, R. B. Xing, Z. Y. Hou, S. S. Huang, and J. Lin, Patterning of light-emitting YVO4:Eu3+ thin films via inkjet printing, J. Phys. Chem. C 114, 9883 (2010). 
[91] J. Delaney, A. Liberski, J. Perelaer, and U. Schubert, A practical approach to the development of inkjet printable functional ionogelsbendable, foldable, transparent, and conductive electrode materials, Macromolecular Rapid Communications 31, 1970 (2010).

[92] F. Loffredo, A. D. Del Mauro, G. Burrasca, V. La Ferrara, L. Quercia, E. Massera, G. Di Francia, and D. D. Sala, Ink-jet printing technique in polymer/carbon black sensing device fabrication, Sens. Actuator B-Chem. 143, 421 (2009).

[93] Y. Wu, E. Girgis, V. Strom, W. Voit, L. Belova, and K. V. Rao, Ultraviolet light sensitive In-doped $\mathrm{ZnO}$ thin film field effect transistor printed by inkjet technique, Phys. Status Solidi A-Appl. Mat. 208, 206 (2010).

[94] X. F. Cui, D. Dean, Z. M. Ruggeri, and T. Boland, Cell damage evaluation of thermal inkjet printed chinese hamster ovary cells, Biotechnol. Bioeng. 106, 963 (2010).

[95] S. Desai, J. Perkins, B. Harrison, and J. Sankar, Understanding release kinetics of biopolymer drug delivery microcapsules for biomedical applications, Mat. Sci. Eng. B-Solid 168, 127 (2010).

[96] C. Menzel, A. Bibl, and P. Hoisington, NIP20: International Conference on Digital Printing Technologies (The Society for Imaging Science and Technology, Salt Lake City, Oct 2004), Vol. 20, pp. 169-175.

[97] Y. M. Chen, R. Mertz, and R. Kulenovic, Numerical simulation of bubble formation on orifice plates with a moving contact line, Int. J. Multiph. Flow 35, 66 (2009).

[98] L. Chun-Fu, L. Wen-Chieh, C. Chun-Jung, and F. Chien-Chung, Microsystems, Packaging, Assembly and Circuits Technology Conference, 2009. IMPACT 2009. 4th International (Institute of Electrical and Electronics Engineers ( IEEE ), Taipei, 2009), pp. 674-677.

[99] H. Elwing, S. Welin, A. Askendal, U. Nilsson, and I. Lundstrom, A wettability gradient method for studies of macromolecular interactions at the liquid/solid interface, J. Colloid Interface Sci. 119, 203 (1987).

[100] V. Hlady, C. Golander, and J. D. Andrade, Hydrophobicity gradient on silica surfaces: A study using total internal reflection fluorescence spectroscopy, Colloids Surf. 33, 185 (1988). 


\section{BIBLIOGRAPHY}

[101] J. Genzer and K. Efimenko, Recent developments in superhydrophobic surfaces and their relevance to marine fouling: a review, Biofouling 22, 339 (2006).

[102] R. Wenzel, Resistance of solid surfaces to wetting by water, Ind. Engin. Chem. 28, 988 (1936).

[103] M. A. Raza, E. S. Kooij, A. van Silfhout, and B. Poelsema, Superhydrophobic Surfaces by Anomalous Fluoroalkylsilane Self-Assembly on Silica Nanosphere Arrays, Langmuir 26, 12962 (2010).

[104] D. Quéré, A. Lafuma, and J. Bico, Slippy and sticky microtextured solids, Nanotechnology 14, 1109 (2003).

[105] B. He, N. A. Patankar, and J. Lee, Multiple equilibrium droplet shapes and design criterion for rough hydrophobic surfaces, Langmuir 19, 4999 (2003).

[106] Y. C. Jung and B. Bhushan, Wetting behaviour during evaporation and condensation of water microdroplets on superhydrophobic patterned surfaces, J. Micros. 229, 127 (2008).

[107] G. McHale, S. Aqil, N. J. Shirtcliffe, M. I. Newton, and H. Y. Erbil, Analysis of droplet evaporation on a superhydrophobic surface, Langmuir 21, 11053 (2005).

[108] M. Sbragaglia, A. M. Peters, C. Pirat, B. M. Borkent, R. G. H. Lammertink, M. Wessling, and D. Lohse, Spontaneous breakdown of superhydrophobicity, Phys. Rev. Lett. 99, 156001 (2007).

[109] F. Mugele and J. Baret, Electrowetting: from basics to applications, J. Phys.: Condens. Matter 17, R705 (2005).

[110] E. Bormashenko, R. Pogreb, G. Whyman, Y. Bormashenko, and M. Erlich, Vibration-induced Cassie-Wenzel wetting transition on rough surfaces, Appl. Phys. Lett. 90, 201917 (2007).

[111] J. Bico, U. Thiele, and D. Quéré, Wetting of textured surfaces, Colloid Surf. A 206, 41 (2002).

[112] N. A. Patankar, On the modeling of hydrophobic contact angles on rough surfaces, Langmuir 19, 1249 (2003).

[113] A. Marmur, Wetting on hydrophobic rough surfaces: To be heterogeneous or not to be?, Langmuir 19, 8343 (2003). 


\section{BIBLIOGRAPHY}

[114] C. W. Extrand, Model for contact angles and hysteresis on rough and ultraphobic surfaces, Langmuir 18, 7991 (2002).

[115] M. Nosonovsky and B. Bhushan, Patterned nonadhesive surfaces: superhydrophobicity and wetting regime transitions, Langmuir 24, 1525 (2008).

[116] S. Moulinet and D. Bartolo, Life and death of a fakir droplet: Impalement transitions on superhydrophobic surfaces, Eur. Phys. J. E 24, 251 (2007).

[117] A. M. Peters, C. Pirat, M. Sbragaglia, B. M. Borkent, M. Wessling, D. Lohse, and R. G. H. Lammertink, Cassie-Baxter to Wenzel state wetting transition: Scaling of the front velocity, Eur. Phys. J. E 29, 391 (2009).

[118] L. Feng, S. H. Li, Y. S. Li, H. J. Li, L. J. Zhang, J. Zhai, Y. L. Song, B. Q. Liu, L. Jiang, and D. B. Zhu, Super-hydrophobic surfaces: from natural to artificial, Adv. Mater. 14, 1857 (2002).

[119] Y. M. Zheng, X. F. Gao, and L. Jiang, Directional adhesion of superhydrophobic butterfly wings, Soft Matter 3, 178 (2007).

[120] J. Y. Chung, J. P. Youngblood, and C. M. Stafford, Anisotropic wetting on tunable micro-wrinkled surfaces, Soft Matter 3, 1163 (2007).

[121] D. Y. Xia, X. He, Y. B. Jiang, G. P. Lopez, and S. R. J. Brueck, Tailoring anisotropic wetting properties on submicrometer-scale periodic grooved surfaces, Langmuir 26, 2700 (2010).

[122] W. Choi, A. Tuteja, J. M. Mabry, R. E. Cohen, and G. H. McKinley, A modified Cassie-Baxter relationship to explain contact angle hysteresis and anisotropy on non-wetting textured surfaces, J. Colloid Interface Sci. 339, 208 (2009).

[123] H. Jansen, H. Gardeniers, M. de Boer, M. Elwenspoek, and J. Fluitman, $A$ survey on the reactive ion etching of silicon in microtechnology, $\mathrm{J}$. Micromech. Microeng. 6, 14 (1996).

[124] X. F. Gao, X. Yao, and L. Jiang, Effects of rugged nanoprotrusions on the surface hydrophobicity and water adhesion of anisotropic micropatterns, Langmuir 23, 4886 (2007).

[125] D. Quéré, Non-sticking drops, Rep. Prog. Phys. 68, 2495 (2005).

[126] B. Bhushan, M. Nosonovsky, and Y. Jung, Nanotribology and Nanomechanics (Springer, Berlin, 2008), pp. 995-1072. 


\section{BIBLIOGRAPHY}

[127] D. Bartolo, F. Bouamrirene, E. Verneuil, A. Buguin, P. Silberzan, and S. Moulinet, Bouncing or sticky droplets: Impalement transitions on superhydrophobic micropatterned surfaces, Europhys. Lett. 74, 299 (2006). 



\section{Summary}

About $71 \%$ of the surface of our planet is covered with water. Our life depends on the presence of water; we can find it all around us. Understanding of the laws that govern the behaviour of liquids is one of the greatest challenges in science. The investigation of the wetting phenomena, i.e.how liquids interact with underlying substrates, is a branch of fluid physics of great interest both from a fundamental as well as an applied points of view. Predicting and modifying of the behaviour of liquids on solid surfaces is important for our everyday life and for numerous industrial applications ranging from food and pharmaceutical industries to automobile manufacturing.

Both smooth and rough, chemically homogeneous and heterogeneous surfaces all influence the spreading of liquid in different ways. In practice, primarily rough chemically heterogeneous surfaces are encountered. Frequently, the surface structures are isotropic, implying that the wetting properties are the same in all directions. For isotropic wetting process, empirical formulas as proposed by Wenzel or Cassie and Baxter are often used to model the most probable liquid static wetting behaviour. As long as the shape of the droplets is approximately spherical, the experimental results are in most cases in good agreement with the models predictions.

A very interesting class of substrates, which are of great interest both from a modelling as well as the application point of views, are anisotropically structured surfaces. These surfaces define a certain direction in which wetting is favourable while inhibiting liquid motion in the others. As an example we refer to the wings of a butterfly on which water droplets can roll-off only away from the butterfly's body. Another example are rice leaves, where the roughly linear surface structure promotes droplets rolling along the length of the leaf. From theoretical point of view, the difficulty arises when one attempts to estimated the static shape of droplets on anisotropic surfaces, as Wenzel and Cassie-Baxter formulas are generally not applicable if the symmetry of liquid spreading is broken. Nevertheless, numerous of publications have appeared in which modified Wenzel and Cassie-Baxter equations are proposed in order to take into account the anisotropy of the underlying substrate. However, when these modified formulas seem to work for the specific surfaces studied in a given publication, attempts to apply to other types of anisotropic structures generally fail. 
As is clear from the discussion above, a systematic study of the static and kinetic behaviour of liquid droplets on anisotropic surfaces should be performed in order to gain a better understanding of the interdependence of the static shape on the underlying structures. As a first step, in this thesis we consider flat chemically patterned surfaces. In this work, the anisotropic patterns chosen consist of stripes of alternating wettability. The stripes are hydrophilic and hydrophobic for the liquids included in these studies. On our type of anisotropic patterns two distinct orthogonal directions of spreading with different properties are present. Specifically, advancing of a liquid is facilitated in the direction parallel to the stripes, when in the direction perpendicular to the stripes motion is opposed by regularly spaced energy barriers created by the hydrophobic stripes. As a consequence of these two modes of spreading, a static shape of droplets can typically be approximated by a cylinder with two hemispherical caps.

However, when the relative widths of the stripes are varied or, the overall macroscopic surface energy is changed, the static shape of the droplets is altered. A systematic study of the variations of the static droplet geometry as a function of the surface energy is presented in Chapter 2. Scaling of the droplet lengths and the static contact angles for different liquids is demonstrated as a function of the relative hydrophobicity of the surface, defined by the ratio of hydrophobic and hydrophilic stripe widths. Furthermore, the Cassie-Baxter equation reproduces correct values of the static contact angles measured in the direction parallel to the stripes; the exact reason for this still remains an open question.

To gain a better insight into the formation of the aforementioned asymmetric droplets, in Chapter 3 we present a high-speed camera study of droplets spreading on stripe-patterned surfaces. An unexpectedly long initial spreading regime is observed, in which the footprint of the droplet remains almost circular and the contact line motion is hardly influenced by the underlying pattern. The experimental results reveal that $65 \%-90 \%$ of the final shape is adopted before the pattern induced spreading occurs. This fact may well be the origin for the scaling reported in Chapter 2. Furthermore, quantitative data relating the velocity of the contact line to the surface energy is extracted, enabling a comparative study of motion in orthogonal directions for different patterns.

In the chapters 4 and 5, the translational motion of a droplet over stripepatterned surfaces is studied. Using well-defined patterns which promote contact line motion along the stripes while confining the advancement perpendicular to the translation direction, it is expected that droplets can move over larger distances as compared to chemically created isotropic 
gradients. In the pattern design, the results obtained in the two preceding chapters are taken into account. To assure the motion will take place only predominantly hydrophilic patterns were used during this study. However, the experimental results have shown that the range of surface energies to be employed can be enlarged.

In the chapter 4 the droplet motion is studied over linear patterns consisting of consecutively placed rectangles made of parallel stripes with increasing surface energy. Such a design is chosen as the droplets adopt the same cylindrical shape during translation as studied in Chapter 2 and Chapter 3 , allowing comparison with the previously obtained data. A well-defined direction of the droplet motion allows using a high-speed camera. As a result, four stages of motion are identified, and quantitative characterisation of the velocities in the various stages as a function of the surface energy is carried out. Furthermore, the accumulated data is discussed to ultimately define guidelines for upcoming designs, depending on which aspect of droplet motion should be emphasized.

In chapter 5 the motion of droplets over radially patterned surfaces is discussed, where the droplet moves away from the centre of the pattern. The radial designs are chosen in view of possible application as antiwetting coatings on nozzle plates to remove unwanted ink wetting layers, which interfere with the printing process. A first difference with linear patterns is that due to the radial symmetry, it is difficult to predict the direction in which the droplet will move. As a result, using high-speed, side-view movies is not possible from an experimental point of view. Second, owing to the considerably smaller confinement of the droplets in the tangential direction, we find that the overall translational velocities are markedly lower. Nevertheless, the motion on radial patterns bears similarities to the linear patterns so the information obtained on linear patterns can be applied in the description of the droplet motion. Furthermore, new features characteristic to radial patterns, which are not observed on linear ones, such as so called liquid "bridges" are identified and discussed.

Finally, in chapter 6 the influence on the droplet static shapes produced by adding shallow structures to our anisotropic striped patterns is discussed. More specifically, the hydrophilic stripes as discussed in all preceding chapters are replaced with $2 \mu \mathrm{m}$ deep grooves, suggesting that solely the Wenzel state, i.e. with the liquid completely filling the underlying structures, is to be expected. However, on a part of the patterns a metastable Cassie-Baxter state, i.e. with the liquid suspended on the groove tops, is observed. The dependence of the final state on the underlying structure, including elongation in the direction parallel to the grooves, is studied as a 
function of groove width. Furthermore, we compare experimental results with the theoretical predictions using available models. Using of calculations for isotropic structures with the same geometric parameters enables a better quantification of the anisotropy induced distortion of the static droplet shapes. 




\section{Samenvatting}

Ongeveer $71 \%$ van het aardoppervlak van onze planeet is bedekt met water. Ons leven hangt af van de aanwezigheid van water; we vinden het overal om ons heen. Begrip van de wetten die het gedrag van vloeistoffen regelen is één van de grootste uitdagingen in de wetenschap. Het onderzoek naar de bevochtigingverschijnselen, bijvoorbeeld hoe de interactie van vloeistoffen is met onderliggende substraten, is een tak van de vloeistof fysica die van groot belang is voor zowel fundamenteel onderzoek als ook voor praktische toegepassingen. Voorspellen en controleren van het gedrag van vloeistoffen op vaste oppervlakken is belangrijk voor ons dagelijks leven en voor talloze industriële toepassingen, variërend van de voedings- en farmaceutische industrie tot de auto-industrie.

Zowel gladde als ruwe, chemisc homogene en heterogene oppervlakken hebben allen op verschillende manieren invloed op de verspreiding van de vloeistof. In de praktijk komen we vooral ruwe chemisch heterogene oppervlakken tegen. Vaak zijn gestructureerde oppervlakken isotroop, wat impliceert dat de bevochtigende eigenschappen hetzelfde zijn in alle richtingen. Voor het isotrope bevochtigingsproces zijn empirische formules, zoals voorgesteld door Wenzel of Cassie en Baxter, vaak gebruikt om het meest waarschijnlijke vloeibare statische bevochtigingsgedrag te modelleren. Zolang de vorm van de druppels ongeveer bolvormig is, zijn de experimentele resultaten in de meeste gevallen in goede overeenstemming met de voorspellingen die voortkomen uit deze modellen.

Een zeer interessante categorie van substraten, die van groot belang is zowel vanuit het oogpunt van de toepassing als ook voor modellering wordt gevormd door anisotrope gestructureerde oppervlakken. Deze oppervlakken definiëren een bepaalde richting waarin bevochtiging gunstig is, terwijl de vloeistof beweging in de andere richting wordt afgeremd. Als voorbeeld verwijzen we naar de vleugels van een vlinder waarop waterdruppels slechts weg kunnen rollen van het lichaam af. Een ander voorbeeld wordt gevormd door rijst bladeren, waar de bih benadering lineaire oppervlakte structuur bevordert dat de druppels in de lengterichting van het blad rollen. Vanuit theoretisch oogpunt blijkt het een uitdaging om de statische vorm van druppels op anisotrope oppervlakken te modelleren, bijvoorbeeld met de formules zoals beschreven door Wenzel en CassieBaxter. Deze modellen zijn over het algemeen niet toepasbaar als de symmetrie van vloeistofspreiding wordt verbroken. Niettemin zijn talloze pub- 
licaties verschenen waarin gemodificeerde Wenzel en Cassie-Baxter worden voorgesteld om rekening te houden met de anisotropie van het onderliggende substraat. Echter, hoewel deze gemodificeerde formules vaak een goede beschrijving lijken te geven voor de specifieke oppervlakken, is het moeilijk om ze algemeen toete passen op andere vormen van anisotrope structuren.

Zoals blijkt uit de discussie hierboven, moet een systematische studie van het statische en kinetische gedrag van vloeibare druppels op anisotrope oppervlakken worden uitgevoerd om een beter begrip te krijgen van de onderlinge afhankelijkheid van de statische vorm op de onderliggende structuren. Als eerste stap beschouwen we in dit poefschrift morphologisch vlakke, chemisch gepatroneerde oppervlakken. In dit werk worden de anisotrope patronen gevormd door strepen me verschillende bevochtigingsgraden. De strepen zijn afwisselend hydrofiel en hydrofoob voor de vloeistoffen die in dit onderzoek zijn bestudeerd. Deze anisotrope patronen definiëren twee verschillende loodrechte richtingen voor verspreiding van de vloeistof: de verplaatsing van de vloeistof in de richting evenwijdig aan de strepen wordt vergemakkelijkt, terwijl in de loodrecht richting wordt tegengewerkt door energie barrières op regelmatige afstanden ten gevolge van de hydrofobe strepen. Als gevolg van deze verschillende modi van spreiding, kan de statische vorm van de druppels doorgaans worden benaderd door een cilinder met twee halfronde uiteindes.

Als de relatieve breedtes van de strepen worden gevarieerd, of, met andere woorden, wanneer de totale macroscopische oppervlakte-energie wor$\mathrm{dt}$ veranderd, heeft dit direct invloed op de statische vorm van de druppels. Een systematische studie van de geometries van de statische druppel geometrie als functie van de oppervlakte-energie wordt beschreven in hoofdstuk 2. We laten zien dat de lengte van de druppel en de statische contacthoeken voor verschillende vloeistoffen schaalt als functie van de relatieve hydrofobiciteit van het oppervlak. Deze laatste grootheid wordt bepaald door de verhouding van hydrofobe en hydrofiele streep breedtes. Bovendien blijkt het Cassie-Baxter model een juiste beschrijving te zijn van de statische contacthoeken, gemeten in de richting evenwijdig aan de strepen. De schaling wordt beschrijven en bediscussieerd

Om een beter inzicht in de vorming van de eerder genoemde asymmetrische druppeltjes te kregen, bestuderen we in hoofdstuk 3 het dynamische gedrag van druppels over de gestreept gepatroneerde oppervlakken met een hoge snelheidscamera. Een onverwacht lang initiële spreidingsregime is waargenomen, waarin het contactoppervlak van de druppel met het substraat bij benadering cirkelvormige blijft en de versprei- 
ding van de vloeistof nauwelijks door het onderliggende patroon wordt beïnvloed. De experimentele resultaten tonen aan dat $65 \%-90 \%$ van de uiteindelijke vorm wordt aangenomen voordat het gestreepte patroon invloed krijgt of de directionele spreiding van de druppel. Dit feit is wellicht wel de reden vovvr het schalingsgedrag zoals gerapporteerd in hoofdstuk 2. Bovendien zijn de kwantitatieve gegevens met betrekking tot de snelheid van de contactlijn in relatie tot de oppervlakte-energie geanalyseerd, waardoor een vergelijkende studie van de beweging in orthogonale richtingen voor verschillende patronen mogelijk wordt.

In de hoofdstukken 4 en 5 wordt de translatie van een druppel over streep-patroon oppervlakken bestudeerd. Goed gedefiniëerde patronen, die beweging langs de strepen bevorderen en tegelijkertijd de verplaatsing loodrecht op de bewegingsrichting beperken, bieden waarschijnlijk de moeglijkheid om druppels over grotere afstanden te verplaatsen ten opzichte van chemisch gedefiniëerde isotrope gradiënten. In het ontwerp van het patroon, wordt rekening gehouden met de resultaten zoals bescreven in de twee voorgaande hoofdstukken. Om er zeker van te zijn dat de beweging daadwerkelijk zal plaatsvinden, werden overwegend hydrofiele patronen gebruikt tijdens deze studie. Uit analyse van de experimentele resultaten blijkt dat het bereik van de oppervlakte-energieën die worden gebruikt, in principe vergroot kan worden.

In hoofdstuk 4 wordt de beweging van druppels bestudeerd over lineaire patronen bestaande uit achtereenvolgens geplaatste rechthoeken, gemaakt van parallel lopende strepen met toenemende oppervlakte-energie. Er is gekozen voor dit ontwerp zodat de druppels dezelfde cilindrische vorm tijdens de beweging aannemen zoals bestudeerd in hoofdstukken 2 en 3, waardoor we de resultaten kunnen vergelijken met de eerder verkregen gegevens. Omdat de bewegingsrichting van de druppel goed gedefiniëerd is, kunnen we gebruik maken van een hoge snelheidscamera. Uit de metingen hebben we vier stadia van de beweging gedentificeerd, en hebben we de snelheden van de contactlijnen in de verschillende stadia als functie van de oppervlakte-energie gekarakteriseerd. Bovendien worden de verzamelde gegevens besproken om uiteindelijk richtlijnen vast te stellen voor toekomstige ontwerpen, afhankelijk van welk aspect van de vloeistofbeweging moet worden benadrukt.

In hoofdstuk 5 wordt de beweging van druppeltjes over radiale patronen besproken, waarbij de druppel van het centrum van het patroon af wordt bewogen. De radiale ontwerpen worden bestudeerd met het oog op de eventuele toepassing als anti-bevochtigingscoatings op 'nozzle'-platen in inkjet printkoppen. Het doel is om ongewenste inkt bevochtigingsla- 
gen, die interfereren met het printproces, te verkomen. Een eerste verschil met lineaire patronen is dat vanwege de radiale symmetrie het moeilijk is om te voorspellen in welke richting de druppel zal bewegen. Als gevolg daarvan is het niet mogelijk gebleken om met onze opstelling hoge snelheidscamera zij-aanzicht filmpjes te maken. Ten tweede, als gevolg van de minder sterke beperking van de beweging loodrecht of de gradient, hebben we gevonden dat over het algemeen de translatiesnelheden lager zijn. Niettemin vertoont de beweging over radiale patronen gelijkenis met de lineaire patronen, zodat de verkregen informatie op lineaire patronen in de beschrijving van de druppel beweging kunnen worden toegepast. Verder worden nieuwe kenmerkende karakteristieken voor radiale patronen, die niet worden waargenomen op lineaire patronen, zoals zogenaamde vloeistof "bruggen" geïdentificeerd en besproken

Tenslotte wordt in hoofdstuk 6 de invloed van relatief ondiepe structuren in onze anisotrope gestreepte patronen op de statische vorm van de druppels bestudeerd. Meer specifiek, de hydrofiele strepen, zoals in alle vorige hoofdstukken besproken, zijn nu vervangen door een groef met een diepte van $2 \mu \mathrm{m}$. Energetische berekeningen op basis van de dimensies van onze structuren suggereren dat alleen de Wenzel toestand kan worden verwacht, die overeenkomt met een volledige bevochtiging van gestructureerde oppervlak door de vloeistof. Echter, op een deel van de patronen wordt een metastabiele Cassie-Baxter toestand waargenomen, waarin de vloeistof de onderliggende structuur niet geheel bevochtigt; de meniscus hangt boven de groeven. De metastabiele eindtoestand van de druppels op de onderliggende structuur, alsmede de verlenging van de druppel in de richting evenwijdig aan de groeven, wordt bestudeerd als functie van met name de groefbreedte. Bovendien vergelijken we onze experimentele resultaten met theoretische voorspellingen op basis van beschikbare modellen. Met behulp van de berekeningen voor isotrope structuren met dezelfde geometrische parameters zijn we in staat om de anisotropie geïnduceerde verstoring van de statische druppel beter te kwantificeren. 




\section{List of publications}

Q Scaling of anisotropic droplet shapes on chemically stripe-patterned surfaces, O. Bliznyuk, E. Vereshchagina, E. S. Kooij, B. Poelsema, Phys. Rev. E, 79, 6, (2009).

(Chapter 2)

Q Initial Spreading Kinetics of High-Viscosity Droplets on Anisotropic Surfaces, O. Bliznyuk, H. P. Jansen, E. S. Kooij, B. Poelsema, Langmuir, 26, 6 (2009).

(Chapter 3)

Q Smart design of stripe-patterned gradient surfaces to control droplet motion, O. Bliznyuk, H. P. Jansen, E. S. Kooij, H. J. W. Zandvliet, B. Poelsema, submitted.

(Chapter 4)

Q Droplet motion over radially patterned surfaces, O. Bliznyuk, V. Veligura, E. S. Kooij, H. J. W. Zandvliet, B. Poelsema, in preparation. (Chapter 5)

Q Metastable droplets on shallow-grooved hydrophobic surfaces, O. Bliznyuk, V. Veligura, E. S. Kooij, H. J. W. Zandvliet, B. Poelsema, Phys. Rev. E, 83, 041607, (2011).

(Chapter 6)

Q Dynamic Dewetting through Micropancake Growth, J. R. T. Seddon, O. Bliznyuk, E. S. Kooij, B. Poelsema, H. J. W. Zandvliet, D. Lohse, Langmuir, 26, 9640 (2010).

(Miscellaneous) 



\section{Acknowledgements}

Four years ago I came to Netherlands to start a project on a completely new for me topic dealing with wettability studies. Looking back over the years, I personally find it to come out extremely good. I find it is a pleasure to thank all those who helped me to adapt to a new life stile, new scientific environment and made this thesis possible.

I would like to thank my promotor Prof. Bene Poelsema for his support and right questions asked at right moments to help me advance with my work.

Special thanks go to my daily supervisor Dr. Stefan Kooij, who was always there to help me with my scientific work as well as with integration in new surroundings. His infallibly good mood and determination with which he overcame an extremely difficult period in his life will always remain an example for me. An extremely big gratitude for his patience in proof-reading my thesis.

Particular thanks to Dr. James Seddon for all discussions we have had during coffee pauses, and for his help whenever I needed it. I would like to thank Herbert for helping me with my experimental setup. Many thanks to Rianne for helping with all administrative papers related issues, and Herman and Hans for making sure everything works smoothly in the laboratory.

I would like to adress my thanks to representatives of our industrial partner Océ Dan Kringus and Marc van der Berg for making it possible for me to use their high-speed camera for data collection as well as making my visit to Océ very pleasant.

I am particularly grateful to my room-mates Waqquar, Patrick and Ahmed and office neighbour Vasilisa for making my working space a very nice place to be. I am grateful to all former and present members of PIN group for all nice moments we had together.

And last but not least, I would like to express my deepest gratitude and thanks to people without whom I would not be here: my parents and my husband Bobby. Without your love, encouragement and support I would have never gone this far. Thank you from the bottom of my heart.

Bliznyuk Olesya

Enschede, April 2011 



\section{CURRICULUM VITAE Olesya Bliznyuk}

Date of Birth: $19^{\text {th }}$ October 1981 In: Krasonodar, Russia

Jun 2007 - present

$2002-2007$

$2000-2002$

$1990-2000$
PhD position, University of Twente (NL)

Experimental study of liquid droplet behaviour on chemically patterned surfaces

(with Prof. Bene Poelsema and Dr. Stefan Kooij)

Master of Sciences in Physics, speciality: Condensed Matter and Nanophysics, Université Louis Pasteur

(B.Sc. and M.Sc. equivalent)

First year of Faculty of Pharmacy, Université Louis Pasteur

Secondary education, Krasnodar, Russia 


